\title{
Fracture toughness evaluations for spent fuel dry storage canister welds and spent fuel clad-pellet structures
}

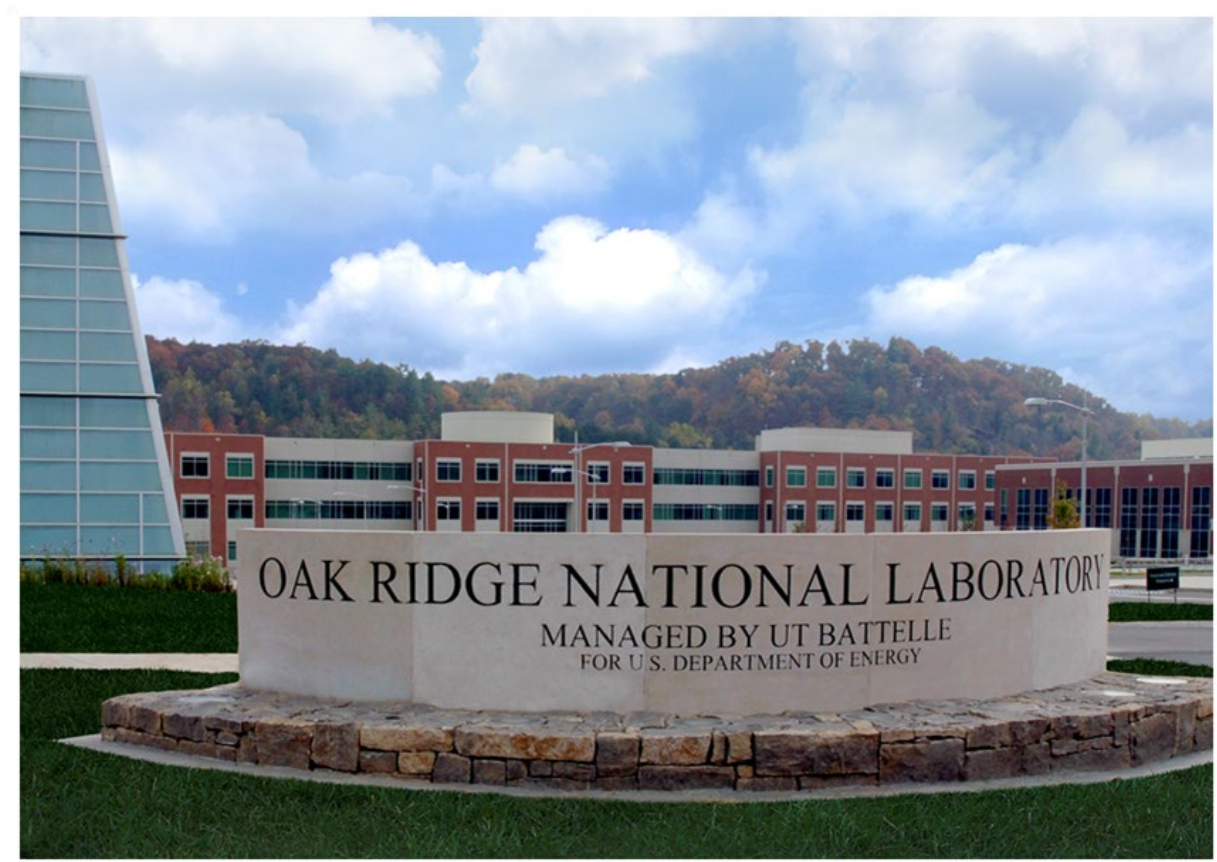

Jy-An Wang

Bruce Bevard John Scaglione Rose Montgomery 


\section{DOCUMENT AVAILABILITY}

Reports produced after January 1, 1996, are generally available free via US Department of Energy (DOE) SciTech Connect.

Website www.osti.gov

Reports produced before January 1, 1996, may be purchased by members of the public from the following source:

National Technical Information Service

5285 Port Royal Road

Springfield, VA 22161

Telephone 703-605-6000 (1-800-553-6847)

TDD 703-487-4639

Fax 703-605-6900

E-mail info@ntis.gov

Website http://classic.ntis.gov/

Reports are available to DOE employees, DOE contractors, Energy Technology Data Exchange representatives, and International Nuclear Information System representatives from the following source:

Office of Scientific and Technical Information

PO Box 62

Oak Ridge, TN 37831

Telephone 865-576-8401

Fax 865-576-5728

E-mail reports@osti.gov

Website http://www.osti.gov/contact.html

This report was prepared as an account of work sponsored by an agency of the United States Government. Neither the United States Government nor any agency thereof, nor any of their employees, makes any warranty, express or implied, or assumes any legal liability or responsibility for the accuracy, completeness, or usefulness of any information, apparatus, product, or process disclosed, or represents that its use would not infringe privately owned rights. Reference herein to any specific commercial product, process, or service by trade name, trademark, manufacturer, or otherwise, does not necessarily constitute or imply its endorsement, recommendation, or favoring by the United States Government or any agency thereof. The views and opinions of authors expressed herein do not necessarily state or reflect those of the United States Government or any agency thereof. 
Materials Science and Technology Division

\title{
Fracture toughness evaluations for spent fuel dry storage canister welds and spent fuel
} clad-pellet structures

\author{
Jy-An John Wang
}

Program Manager

Bruce Bevard ${ }^{a}$, John Scaglione ${ }^{a}$, Rose Montgomery ${ }^{a}$

${ }^{a}$ Reactor \& Nuclear Systems Division

Date Published: February 2021

Prepared by

OAK RIDGE NATIONAL LABORATORY

Oak Ridge, TN 37831-6283

managed by

UT-BATTELLE, LLC

for the

US DEPARTMENT OF ENERGY

under contract DE-AC05-00OR22725 
This page is intentionally blank 


\section{Contents}

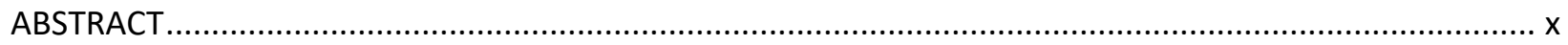

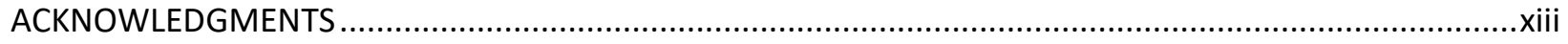

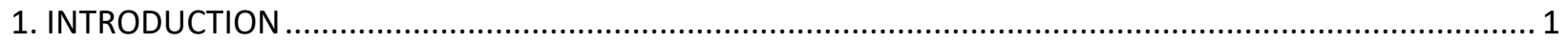

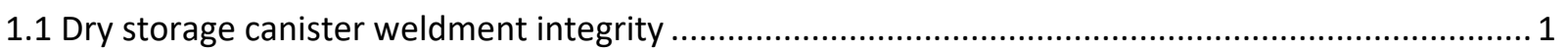

1.2 Spent nuclear fuel clad-pellets structure integrity ....................................................................... 1

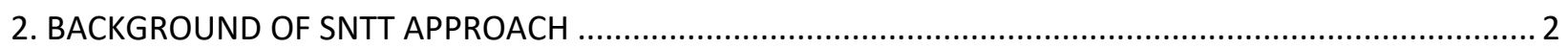

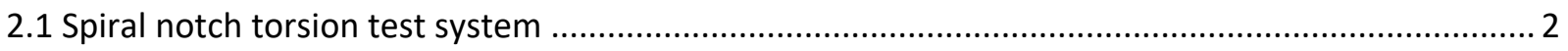

2.2 Non-coplanar crack front finite element analyses...................................................................... 4

2.2.1 SNTT finite element modeling methodology for ductile materials ........................................ 5

2.2.2 The Evolution of SNTT Compliance and Fracture Resistance for Ductile Materials .................. 6

2.2.3 Experimental verification on the developed SNTT fatigue crack compliance protocol............. 11

2.2.4 J-Integral analysis development for solid rod SNTT specimens ............................................... 12

2.3 Typical SNTT experimental procedures and results..................................................................... 15

3. BIAXIAL TESTER DEVELOPMENT TO PERFORM SNTT TESTING PROTOCOL ............................................ 16

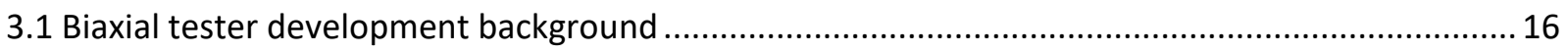

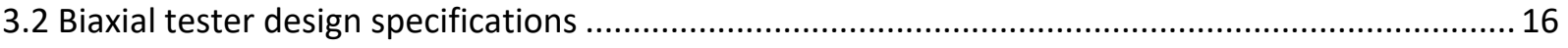

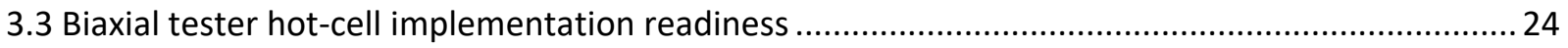

4. FRACTURE TOUGHNESS EVALUATION FOR SNF DRY STORAGE CANISTER WELD ................................28

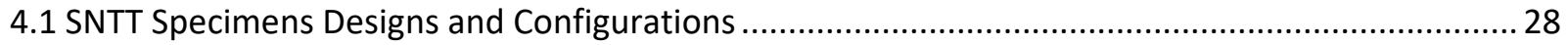

4.2 SS304/308 Weld SNTT Sample Fixture Design Configuration....................................................... 30

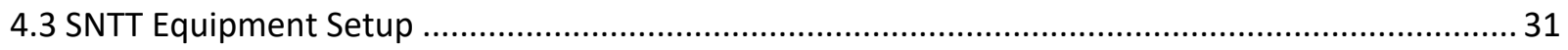

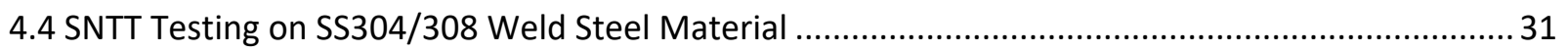

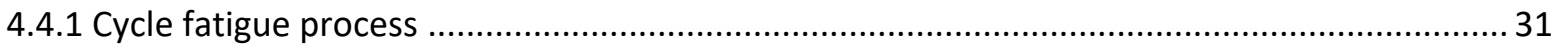

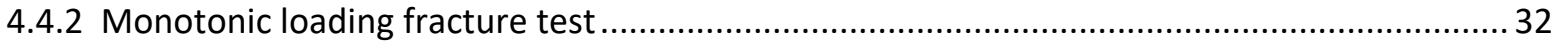

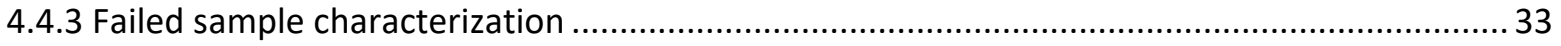

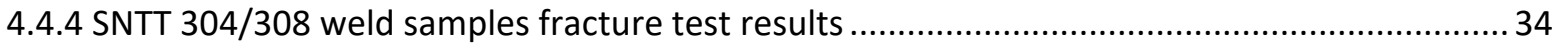

4.5 Fracture toughness evaluation on the tested SNTT weld samples............................................. 35

4.5.1 FEM analyses and energy release rate evaluation for SNTT Weld-10 specimen test results.... 35

4.5.2 J-Integral evaluation for SS 304/308 weldment material .................................................... 37

4.5.3 J-integral evaluation comparison between SNTT and Compact Tension (CT) tests results....... 38

5. FRACTURE TOUGHNESS EVALUATION FOR THIN-SHELL CLAD-PELLET INSERTS STRUCTURE..................40

5.1 Mode I + Mode III mixed-mode fracture toughness evaluation ...................................................... 40 
5.2 Zr-4 clad SNTT specimen preparation and biaxial tester set-up ................................................ 41

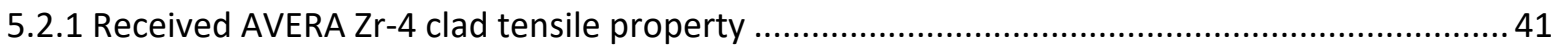

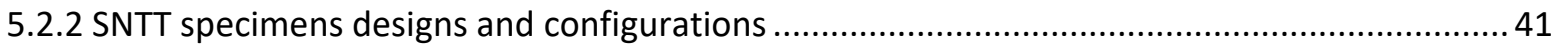

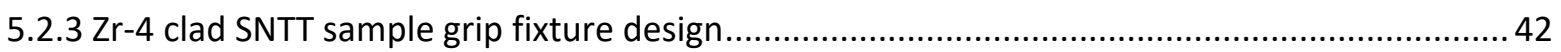

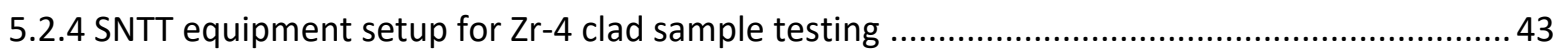

5.2.5 Pilot fatigue pre-crack testing on Zr-4 clad and SS clad SNTT specimens................................44

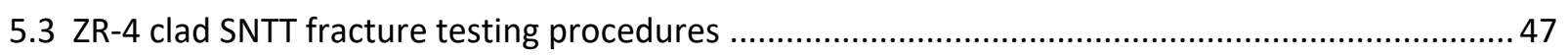

5.3.1 Zr-4 clad SNTT sample cycle fatigue testing process .......................................................... 47

5.3.2 Zr-4 clad SNTT sample monotonic loading fracture test ...................................................... 47

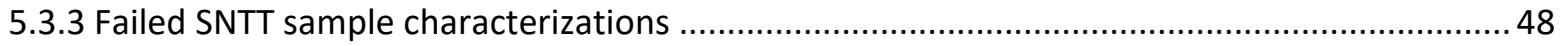

5.3.4 Zr-4 clad SNTT samples fracture test results ..................................................................... 50

5.4 ZR-4 clad SNTT sample fracture toughness evaluation............................................................. 51

5.4.1 Typical SNTT finite element modeling methodology for ductile materials ............................51

5.4.2 Energy release rate evaluation for Zr-4 clad SNTT sample with short and medium crack length

5.4.3 Energy release rate evaluation for Zr-4 clad SNTT sample with long crack length ..................56

5.4.4 Fracture toughness evaluation of the tested Zr-4 clad SNTT samples ..................................57

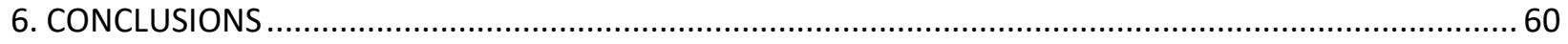

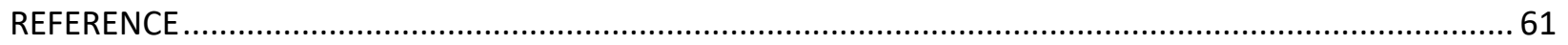

APPENDIX A: TESTRESOURCE ELECTRODYNAMIC SYSTEM USER MANUAL...........................................613 


\section{Lists of Figures}

Fig. 1. SNTT test sample set-up, including the biaxial extensometer.

Fig. 2. Pure torsion loading schematic indicates that principal tensile stress profile is perpendicular to the 3-D spiral curve at $45^{\circ}$ pitch angle.

Fig. 3. Fractured 7475-T7351 aluminum SNTT specimen indicates the crack propagation orientation is perpendicular and toward the cylinder central axis.

Fig. 4. SNTT FEA Models (Left) used for brittle materials where no fatigue pre-crack required, and (Right) used for ductile materials where deep crack is needed to increase the crack front constraint.

Fig. 5. (Left) Typical finite element models used for ductile material fracture toughness characterization, due to high ductility of the 304/308 weld material, the singular wedge element with quarter-node element was relaxed back to normal wedge element with middle-node element; (Right) Typical FEM analyses results that indicate tri-axial tensile stress at near crack tip and butterfly plastic process zone around the crack tip, which indicate a high geometry constraint toughness testing configuration.

Fig. 6. (a) The evolution of crack growth in the SNTT sample with FEA simulation models. (b) Single crack depth example FEA displacement results, for $a / D=0.10, D=0.375$-inch, Steel, under end rotation theta $=0.002$ with ends fixed at $\mathrm{U} 1$ and $\mathrm{U} 2$ orientations.

Fig. 7. (a) The unscaled compliance evolution with the crack growth; (b) The scaled compliance evolution along the crack growth with unbroken ligament factor.

Fig. 8. (a) The unscaled energy release rate evolution with the crack growth; (b) The scaled energy release rate evolution along the crack growth with unbroken ligament factor.

Fig. 9. The failed B2 specimen of X52 baseline sample, (a) entire view; (b) enlarged view of the middle section of tested SNTT sample, (c) the fatigue pre-crack growth profile of X52 weld SNTT sample W2, where the crack

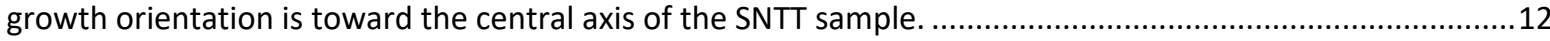

Fig. 10. The fractured profiles of tested SNTT A302B specimen. ................................................................15

Fig. 11. Plot of SNTT test results from (a) load cell and strain gauge, and (b) load cell and biaxial extensometer...15

Fig. 12. TestResource Axial/Torsion biaxial tester design configurations.........................................................23

Fig. 13. Geometry details of the SS304/308 weld steel SNTT specimen. ..........................................................28

Fig. 14. SNTT specimens with different spiral groove designs, (Top) single loop spiral groove with $0.0375^{\prime \prime}$ notch depth, (Middle) single loop with 0.100" notch depth, (Bottom) two loops with 0.1125" notch depth............29

Fig. 15. SNTT weld sample machining physical location in the as-received Sandia weldment. ...............................29

Fig. 16. SNTT HAZ sample machining physical location in the as-received Sandia weldment..................................30

Fig. 17. Schematic for fixture to adapt the $304 / 308$ weld SNTT specimen. ......................................................30

Fig. 18. Schematic for base to adapt the SS304/308 weld SNTT fixture.........................................................31

Fig. 19. HAZ-1 test results, (Left) upon sudden failure a significant shock reaction was observed, (Right) The slopes of different loading and unloading sequences does not change, indicate no crack growth during the monotonic loading, the specimen undergo significant non-linear deformation before final failure, at $56 \mathrm{~N}-\mathrm{m}$ (495 Lbf-in) fracture torque.

Fig. 20. 2-loop Weld-4 test results, (Left) upon sudden failure a significant shock reaction was observed, (Right) The slopes of different loading and unloading sequences does not change, indicate no crack growth during the monotonic loading, the specimen undergo significant non-linear deformation before final failure, at $41 \mathrm{~N}$ -

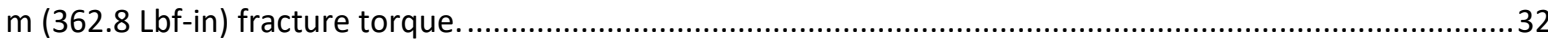

Fig. 21 SNTT HAZ-1 fracture specimen and the associated fracture surface profile .............................................33

Fig. 22. SNTT 2-loop Weld-10 fracture specimen and the associated fracture surface profile, the red arrows point to the fatigue pre-crack front.

Fig. 23. (Left) Most SNTT weld 1-loop shallow-notch specimens' failures are initiated at HAZ regions, (Right) All the weld 2-loop deep-notch specimens are failed at HAZ regions.

Fig. 24. SNTT Weld-9 specimen fracture test results, (Left) Torque vs. angle data, and (Right) Upon failure significant axial load shock was observed, reaching magnitude of $\sim 1800 \mathrm{~N}$. 
Fig. 25. Finite element model for SNTT Weld-10 specimen test simulation.

Fig. 26. (Left) Full model FEM deformation and von-Miss stress profile, (Right) Deformation and von Miss stress profiles at near middle layer of finite element model.

Fig. 27. SNTT test torque load versus rotation angle trend curve up to maximum fracture torque ..........................38

Fig. 28. (Left) Opening tensile mode I observed from $45^{\circ}$ pitch angle spiral cut slit; (Right) Out-of plane shear mode III observed from $0^{\circ}$ pitch angle cut slit (Along axial orientation) under pure torsion. ..................................41

Fig. 29. A uniaxial tensile tube test and tensile stress-strain curve for received $\mathrm{Zr}-4$ cladding..............................42

Fig. 30. Geometry details of SNTT specimen made of $\mathrm{Zr}-4 \mathrm{clad}$ and alumina pellets............................................42

Fig. 31. (Left) SNTT specimen with a short $45^{\circ}$ notch, (Right) SNTT specimen with a 0.762-mm through clad

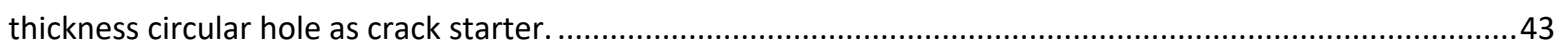

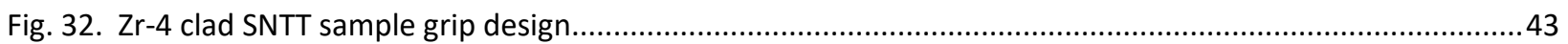

Fig. 33. (Left) SNTT biaxial tester set-up, (Right) SNTT clad specimen installation into biaxial tester torque ends, and SNTT sample made of $\mathrm{Zr}-4 \mathrm{clad} /$ pellets rod and end-grips system. ...........................................................44

Fig. 34. The fatigue crack growth profile of Zr-4 clad SNTT sample under $11.3 \mathrm{~N}$-m torque cyclic loading..............45

Fig. 35. (a) SS304 clad SNTT sample with 0.762-mm circular hole as crack starter; (b) under cyclic fatigue the circular hole elongated into 1.524-mm elliptical hole; (c) final fatigue crack growth on SNTT sample.

Fig. 36. (a) Fatigued failure pre-hydride Zr-4 clad SNTT sample with through thickness hole as crack starter, (b) which shows a brittle fracture characteristic w/o obvious fatigue pre-crack growth profile.

Fig. 37. Zr4-N3 sample test results, (Left) upon sudden failure a significant axial shock reaction force was observed, (Right) The slopes of different loading and unloading sequences does not change, indicate no crack growth during the monotonic loading, the specimen failed at $21 \mathrm{~N}$-m torque.

Fig. 38 (a) Three medium crack length Zr-4 clad SNTT samples fractured surface profiles, and (b) the detailed fracture surface profile beyond notch crack starter at fracture initiation.

Fig. 39. Fractured Zr-4 clad SNTT samples of 9.525-mm diameter, (Left) a through thickness pin hole with shallow surface wing of $1.5-\mathrm{mm}$ in length along $45^{\circ}$ slope as crack starter, (Right) a through thickness pin hole with shallow surface wing of $1.5-\mathrm{mm}$ in length along $30^{\circ}$ slope as crack starter. Both types of the fatigued SNTT samples show that fatigued crack front and the final fracture profiles are aligned with the $45^{\circ} \mathrm{spiral} \mathrm{crack}$ front that is normal to the principal tensile stress contour profile.

Fig. 40. (Left) Fracture profile of the Zr-4 clad SNTT specimen with long fatigue pre-crack length (Right) Detailed crack front view shows crack initiation direction is deviated from the $45^{\circ}$ spiral crack front, which indicates a mixed mode failure mechanism (Mode I + Mode III) under SNTT testing protocol.

Fig. 41. (a) Clad-pellet FEM mesh profile, (b) Schematic diagram of crack seam and crack fronts profiles..............53

Fig. 42. FEM deformation of short crack SNTT sample and the associated von-Mises stress contours profile. .........55

Fig. 43. FEM deformation of medium crack SNTT sample and the associated von Mises stress contours. ..............55

Fig. 44. (a) FEM deformation of long crack SNTT sample and associated von-Mises stress contours profile; (b) The detailed von Miss stress contour view at crack tip. 


\section{Lists of Tables}

Table 1 SNTT 304/308 weld specimens and 304 base specimen fracture test results .............................................34

Table 2 SNTT 304/308 weld \& SS304 base specimens fracture toughness test results ..............................................37

Table 3 Summary of fracture toughness obtained from SNTT test and CT test results..............................................39

Table 4 Summary of Zr-4 clad SNTT samples fracture test results....................................................................50

Table 5 Zr-4 clad mechanical property for Abaqus elastic-plastic analyses input...................................................53

Table 6 FEM geometry, mesh, and clad-pellet contact criteria for Abaqus modeling input ...................................54

Table 7 Summary of fracture toughness obtained from SNTT tests with medium crack length ...............................58

Table 8 Summary of mixed-mode fracture toughness obtained from SNTT tests with long crack length .................59 


\begin{abstract}
Integrity of spent nuclear fuel (SNF) interim storage canisters is very important to the safety of the backend nuclear fuel cycle. Stress corrosion cracking (SCC) potential of interim storage canister has been considered as a high priority. Because no post-weld heat treatment was required for forming these canisters, the high tensile residual stress existed within these canister welds. This can change the fracture resistance capacity significantly as well as increase SCC potential. Due to relative thin shell thickness of a canister weldment, the spiral notch torsion test (SNTT) method was used to estimate the canister weldment fracture toughness. SNTT was developed to measure the intrinsic fracture toughness of structural materials using small specimens. The SNTT method has been applied to a wide variety of structural materials, such as low-alloy steels, stainless steel, aluminum alloy, ceramics, concrete, and composites. The SNTT system operates by applying pure torsion to cylindrical specimens with a notch line that spirals around the specimen at a $45^{\circ}$ pitch. In order to carry out pure torsion load mode, biaxial tension/torsion tester was developed accordingly to perform SNTT protocol.
\end{abstract}

Moreover, applying fracture mechanics approach to SNF system reliability investigation is warranted due to the inherent flaws and hydride structures existed in a SNF system after nuclear reactor operation. However, none of the existing fracture toughness data deal with fuel cladding specific geometry or spent fuel material conditions, such as cladding structure with the pellet-inserts and the associated pellet clad mechanical interactions induced mixed-mode damage mechanisms. Thus, the development of an intrinsic fracture mechanics approach that is suitable for SNF materials is needed. Furthermore, due to thin wall and small dimension of clad tubing structure, the SNTT method was used to estimate the clad tubing structure fracture toughness.

Fracture testing were performed on the received stainless steel canister weldment, most SNTT weld samples fracture initiation sites are at heat-affected zone (HAZ) regions. The estimated fracture toughness $\mathrm{J}_{\mathrm{Q}}$, for the baseline SS304 steel is at $283 \mathrm{~kJ} / \mathrm{m}^{2}$. The estimated $\mathrm{J}_{\mathrm{Q}}$, for the $\mathrm{SS} 304 / 308$ weld and baseline metals are $148 \mathrm{~kJ} / \mathrm{m}^{2}$ and $459 \mathrm{~kJ} / \mathrm{m}^{2}$, respectively.

Out of cell fracture testing for spent fuel structure were carried out on the surrogate rods made of $\mathrm{Zr}-4$ clad and alumina inserts, the estimated fracture toughness values for baseline $\mathrm{Zr}$-4 cladding with aluminapellet inserts are: (1) For SNTT samples with a short or medium crack length, between 5.4-mm and 8$\mathrm{mm}$, the estimate $\mathrm{J}_{\mathrm{IQ}}$ upon fracture initiation for the baseline $\mathrm{Zr}-4$ cladding is at $50 \mathrm{~kJ} / \mathrm{m}^{2}$ with 2-sigma uncertainty of $3.26 \mathrm{~kJ} / \mathrm{m}^{2}$, and the associated $\mathrm{K}_{\mathrm{IQ}}$ is at $67.46 \mathrm{MPa} \sqrt{\mathrm{m}}$; and (2) For SNTT samples with a long crack length, around $13-\mathrm{mm}$, the crack initialization is deviated from that of the Mode-I tensile 
fracture and appears to be a mixed-mode fracture of Mode I - tensile stress and Mode III - out of plane shear stress; the estimated $\mathrm{J}_{\mathrm{MQ}}$ is at $18.9 \mathrm{~kJ} / \mathrm{m}^{2}$, the associated $\mathrm{K}_{\mathrm{MQ}}$ is at $41.4 \mathrm{MPa} \sqrt{\mathrm{m}}$. 
This page is intentionally blank 


\section{ACKNOWLEDGMENTS}

This research was jointly sponsored by the US Department of Energy (DOE) Used Fuel Disposition Campaign (UFDC) under DOE contract DE-AC05-00OR22725 with UT-Battelle, LLC. The authors thank ORNL program managers, Bruce Bevard, John Scaglione, and Rose Montgomery, for their continued support and guidance during the program development; Chris Herbst for developing axial/torsion biaxial tester, Randy Parton for preparing the clad specimens and the associated specimen end-grips; Charles Bryan for providing Sandia Mock-up canister weldment, Doug Stringfield for preparing weld specimens, Tom Geer for providing etching surface images of the received weldment. 
This page is intentionally blank 


\section{INTRODUCTION}

\subsection{Dry storage canister weldment integrity}

Stress corrosion cracking (SCC) of interim storage canisters [1] has been identified as a high priority. Due to cold working and welding, high tensile weld residual stresses (WRS) exist in the canister weld metal. WRS are function of weld geometry, sample thickness, base metal properties, and welding procedures. Thus, WRS are specific and can only be effectively measured from a mockup weld formed with the identical procedures as that of the real canisters. Weld residual strains have been measured by neutron diffraction and interpreted in terms of residual stresses in a stainless steel (SS) longitudinal welded plate removed from a Sandia mockup canister [2]. The general observations from the neutron residual stress mapping study on the received Sandia mockup weld plates are: The maximum tensile residual stress of $439 \mathrm{MPa}$, located in fusion zone (FZ) orientated in the axial direction, is much higher than the yield stress of $200 \mathrm{MPa}$ for the 304L and 308L SS metals; and it is expected to above the yield stress of the weld. Furthermore, based on Lincoln SAW 308/308L weld [3,4], the yield stress and UTS for 308/308L weld are $380 \mathrm{MPa}$ and $565 \mathrm{MPa}$, respectively. The residual axial tensile stress also indicates that tensile stresses exist throughout the thickness of the weldment in the FZ, including HAZ region up to 10-mm away from the weld toe (i.e., the edge of FZ), and the associated maximum axial tensile residual stress is at $272 \mathrm{MPa}$ [2]. Typically, weld FZ has the highest residual stresses; however, the HAZ regions is most likely to trigger the fracture due to relatively low fracture toughness, or to initiate SCC due to sensitization from the welding process.

Because no post-weld heat treatment was required for forming these canisters, the high tensile residual stress existed within these received canister welds. This can change the fracture resistance capacity significantly as well as increase SCC potential. Due to relative thin shell thickness of a canister weldment, the spiral notch torsion test (SNTT) method of small specimen approach was used to estimate the canister weldment fracture toughness. For the ductile SS weldment material, a path-independent J-integral method, that can characterize the fracture behavior for the elastic-plastic and fully plastic conditions, was used for fracture toughness evaluation [5-7].

\subsection{Spent nuclear fuel clad-pellets structure integrity}

Fracture mechanics approach in applying to spent nuclear fuel (SNF) system reliability investigation, especially for the high burn-up (HBU) SNF, during SNF long term dry storage or SNF transportation is necessary. This is because inherited flaws and inhomogeneous structures existed in a SNF system from the nuclear reactor operation, such as hydride and oxide formation, surface flaw induced by SNF 
assembly contact interactions, or internal flaws induced by pellet-clad mechanical interaction (PCMI). Through-thickness crack propagation and fracture toughness of hydride zirconium alloys has been studied [8-11]; it appears that hydride content/profile has a significant effect on zirconium alloys fracture toughness. However, none of the existing fracture toughness data deal with fuel cladding specific geometry or SNF material conditions, such as cladding structure with the segment fuel pellet inserts configuration and the potential PCMI induced mixed-mode failure mechanisms. Consequently, the application of existing fracture toughness data to SNF system reliability investigation can be error prone; thus, the development of an in-situ fracture mechanics approach for SNF materials is needed.

The objective of SNF toughness research is to develop an in-situ fracture toughness testing protocol, including the associated analytical procedure that is suitable for evaluating the SNF system fracture toughness. Due to thin wall and small diameter of a Zr-4 cladding structure, the SNTT method was used to carry out the clad-pellet structure fracture toughness tests. For the ductile $\mathrm{Zr}-4$ cladding material, a path-independent J-integral method was used for fracture toughness evaluation.

\section{BACKGROUND OF SNTT APPROACH}

Adequate toughness is an essential attribute of structure systems design. American Society for Testing and Materials (ASTM) standard test methods, Standard Test Method for Plane-Strain Fracture Toughness of Metallic Materials (E399), are widely used to determine fracture toughness of metallic materials, using compact tension and compact disk tension specimens having thickness and volume sufficient to ensure the plane-strain condition at the crack front. Meeting the requirements is difficult and impractical because structure systems materials to be investigated may be geometrically unsuitable and/or have insufficient volume for making the standard specimen. Therefore, use of small specimens for fracture toughness measurement is essential, and SNTT approach was developed to meet this criterion. Despite the international efforts on the development of small specimen testing techniques, no methods currently exist for direct measurement of fracture toughness from small specimens without a concern for size effect. Unlike the conventional test methods, the SNTT method is capable of testing small rod specimens that bear no resemblance to conventional compact tension specimens nor using conventional mode of loading.

\subsection{Spiral notch torsion test system}

SNTT servo-hydraulic biaxial test system (Fig. 1) was developed for determining fracture toughness values for a wide spectrum of materials ranging from ductile to brittle materials. Test results obtained for 
steel, aluminum, stainless steel, graphite and mullite ceramic were compared and validated with those reported in the open literature [12-20].

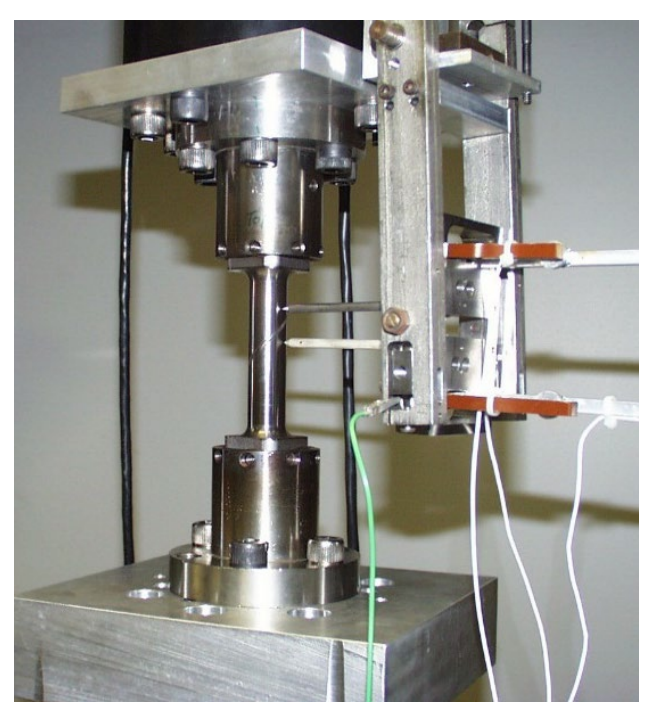

Fig. 1. SNTT test sample set-up, including the biaxial extensometer.

The SNTT test system operates by applying pure torsion to cylindrical specimens having a notch line that spirals around the specimen at a $45^{\circ}$ pitch angle. The pure torsion creates a uniform equibiaxial tension/compression stress field on each of concentric cylinders and the grooved line effectively becomes a Mode I crack mouth opening. It is not difficult to visualize that the rod specimen is a different manifestation of a compact-tension specimen having a thickness equivalent to the total length of the spiral notch. Compact-tension specimen testing lacks a method to uniformly distribute applied load throughout the entire specimen thickness because the stresses at and near the two free surfaces are anomalous, resulting in shear lip formation often discernible in fractured specimens. In contrast, the torque load acting on every cross-section along the rod specimen is the same and directly measurable. A plane-strain condition is achieved on every plane normal to the spiral groove. Because of the plane strain and axisymmetric constraint and the uniformity in the stress and strain fields, the crack front must propagate perpendicularly toward the specimen axis along the conoids (Fig. 2). Post-mortem examination verified the crack propagation behavior (Fig. 3). 


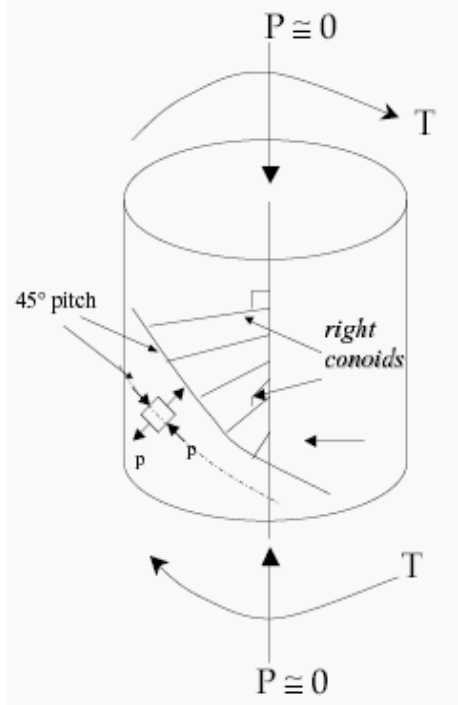

Fig. 2. Pure torsion loading schematic indicates that principal tensile stress profile is perpendicular to the 3-D spiral curve at $45^{\circ}$ pitch angle.

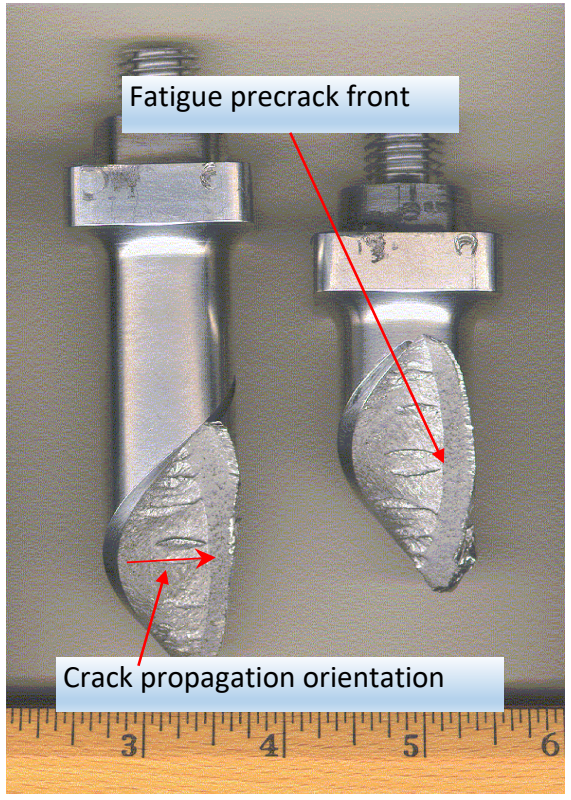

Fig. 3. Fractured 7475-T7351 aluminum SNTT specimen indicates the crack propagation orientation is perpendicular and toward the cylinder central axis.

\subsection{Non-coplanar crack front finite element analyses}

Due to non-coplanar crack front of SNTT configuration and the lack of close form solutions, fracture toughness of SNTT method was evaluated using 3-D finite element analysis and $J$-integral approach. 
Typically, a finite element model shown in Fig. 4a is used for brittle SNTT specimens with shallow crack front and Fig. 4b is for ductile specimens with deep crack front. The SNTT FEA model normally contains

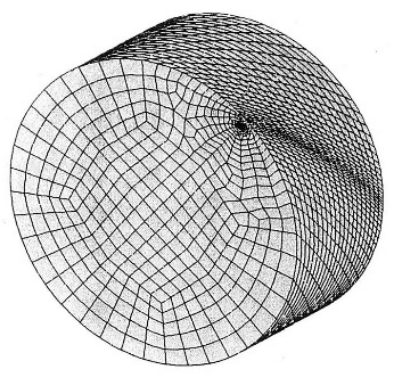

(a) Shallow crack

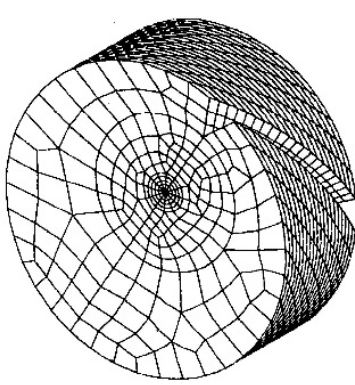

(b) Deep fatigue precrack

Fig. 4. SNTT FEA Models (Left) used for brittle materials where no fatigue pre-crack required, and (Right) used for ductile materials where deep crack is needed to increase the crack front constraint.

20-node quadratic brick elements with reduced integration and singular wedge element along crack front. The ABAQUS code was used to simulate the 3-D spiral crack front and crack propagation orientation during transition phases between fatigue crack growth and the final fracture under pure torsion loading, a governing equation for estimating fatigue crack penetration depth was also developed accordingly [21].

\subsubsection{SNTT finite element modeling methodology for ductile materials}

The methodology used for developing finite element model (FEM) and the typical FEM analyses results are illustrated in Fig. 5. The FEM designed for the ductile material SNTT fatigue pre-crack sample characterization was used for demonstration. Due to high ductility of the 304/308 weld material, the singular wedge element with quarter-node elements around crack tip was relaxed back to normal wedge element with middle-node elements. The typical FEM analyses results are also shown in Figure 32, where the tri-axial tensile stress profiles and the butterfly plastic process zone indicate a high geometry constraint condition exists in the proposed SNTT fracture toughness testing protocol. 

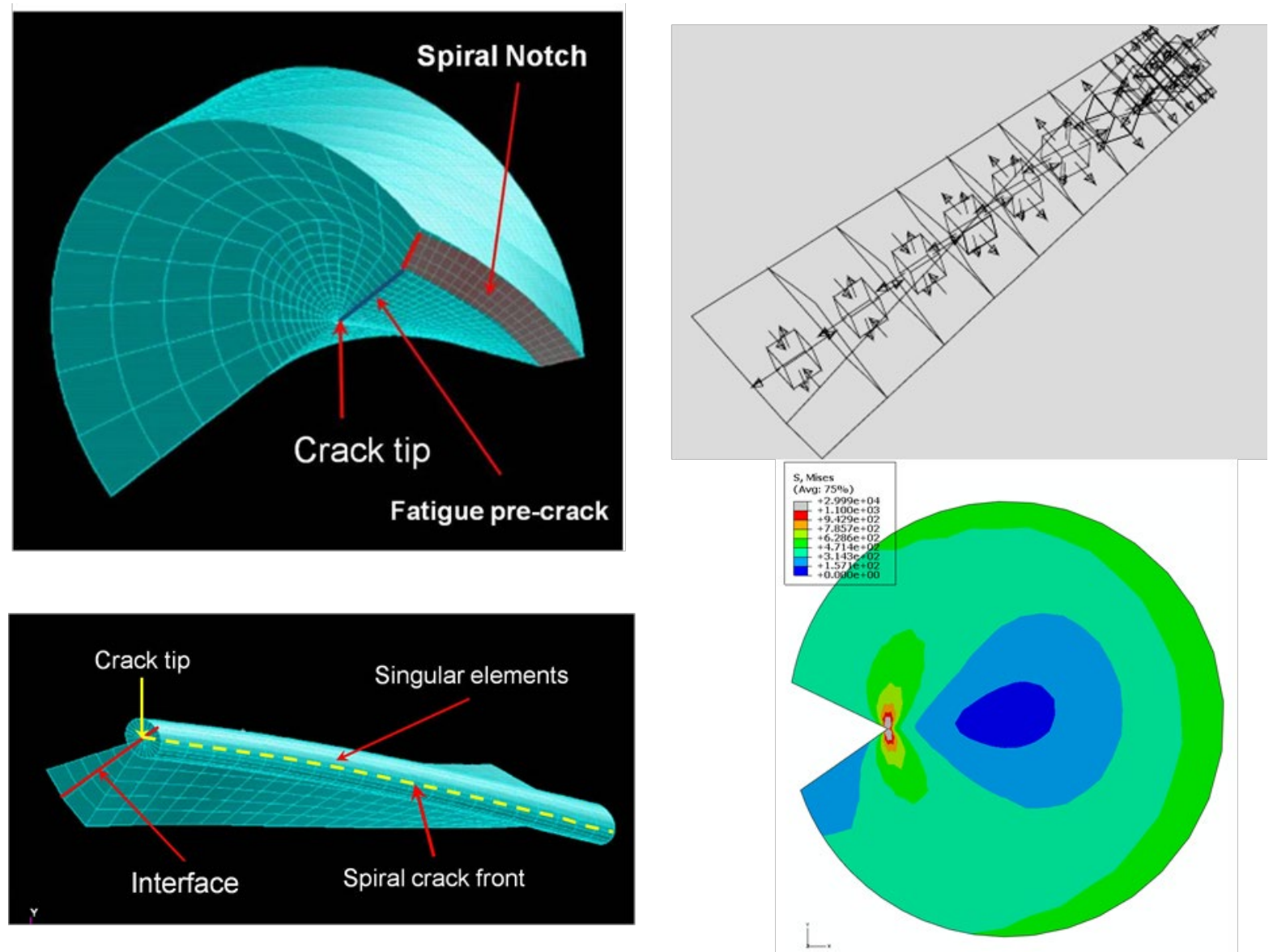

Fig. 5. (Left) Typical finite element models used for ductile material fracture toughness characterization, due to high ductility of the 304/308 weld material, the singular wedge element with quarter-node element was relaxed back to normal wedge element with middle-node element; (Right) Typical FEM analyses results that indicate triaxial tensile stress at near crack tip and butterfly plastic process zone around the crack tip, which indicate a high geometry constraint toughness testing configuration.

\subsubsection{The Evolution of SNTT Compliance and Fracture Resistance for Ductile Materials}

Development of SNTT fatigue crack growth protocols in addition to single notch-front geometry is essential for SNTT method in applying to ductile materials. The crack growth behavior of SNTT testing protocols has been effectively established using an integrated experimental, numerical and analytical approach, as illustrated in this section. The results indicate that the proposed protocol not only provides a significant advance in understanding the compliance evolution of the SNTT specimen, but also can be readily utilized to assist future developments in engineering structural materials performance reliability investigation. 
Systematic studies show that the SNTT-type specimen can effectively simulate the behavior of a thick compact-tension specimen with thickness equal to the spiral groove length of the SNTT sample. Finite element simulations were performed to obtain the energy release rates at different crack lengths (depth) with the corresponding torques and rotation angles. By summarizing these numerical results, the evolution of the SNTT compliance and the energy release rates were studied with respect to different crack lengths. Two non-dimensional indices, the characteristic compliance and the characteristic energy release rates of SNTT, were proposed to quantify the crack growth process of SNTT. Collapse trends were observed between SNTT samples with different dimensions, as well as between samples made from both steel and aluminum. Analytical models in both broken and unbroken ligament forms were proposed to quantify the crack penetration depth based on these non-dimensional indices. The sensitivity in the broken-ligament form facilitates the experiment measurements, which could be easily adapted by industrial communities.

Fig. 6 (a) shows the systematic evolution of the crack growth in the SNTT sample with FEA simulations; where the diameter of the cylinder is $D$, and $a$ is the crack length. The ratio of crack length over diameter increases from 0.10 to 0.45 . The length of each model is the same during the crack growth process. Fig. 6 (b) shows the FEA deformation results for $a / D=0.1$ under end rotation of 0.002 radian [21]. Based on FEA simulations and experimental verification input, the evolution of the compliance function and energy release rate were developed as shown in Fig. 7 and Fig. 8.

Detailed studies show that the evolutions of compliance and fracture resistance of the SNTT sample during the crack growth process can be unified together irrespective of specimen sizes and material types. The evolutions of compliance and fracture resistance in SNTT process were formulated with simple compliance governing equations as function of the ratios of crack lengths vs. the cylindrical diameter.

The finite element simulations were established with different crack lengths for selected SNTT specimens verified by physical measurements. Steel and aluminum SNTT specimens were selected with two diameters of 1.0 inch and 0.375 inch for analysis. The objective was to obtain the evolutions of both compliance and the fracture resistance during the crack growth process for different types of SNTT samples. 

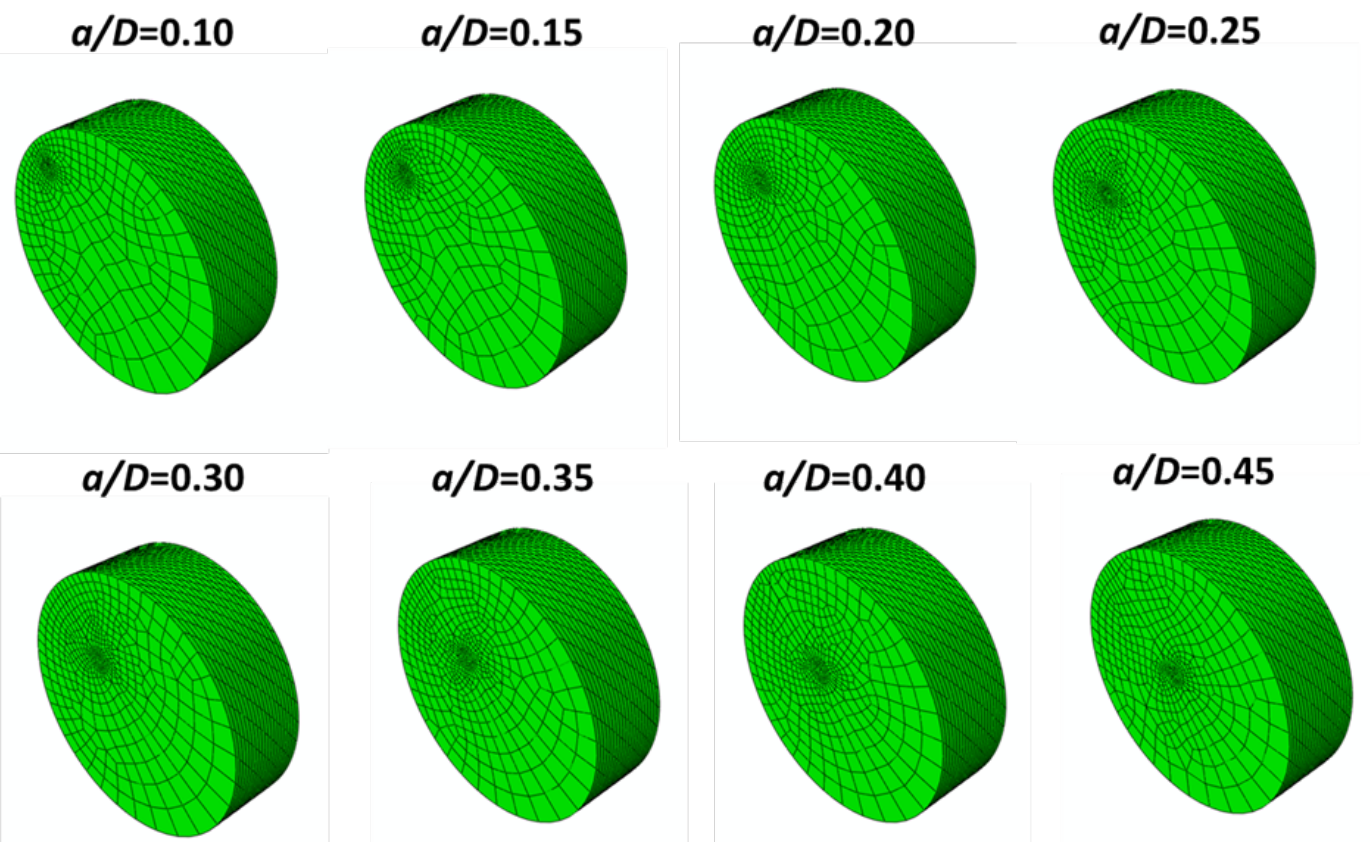

(a)
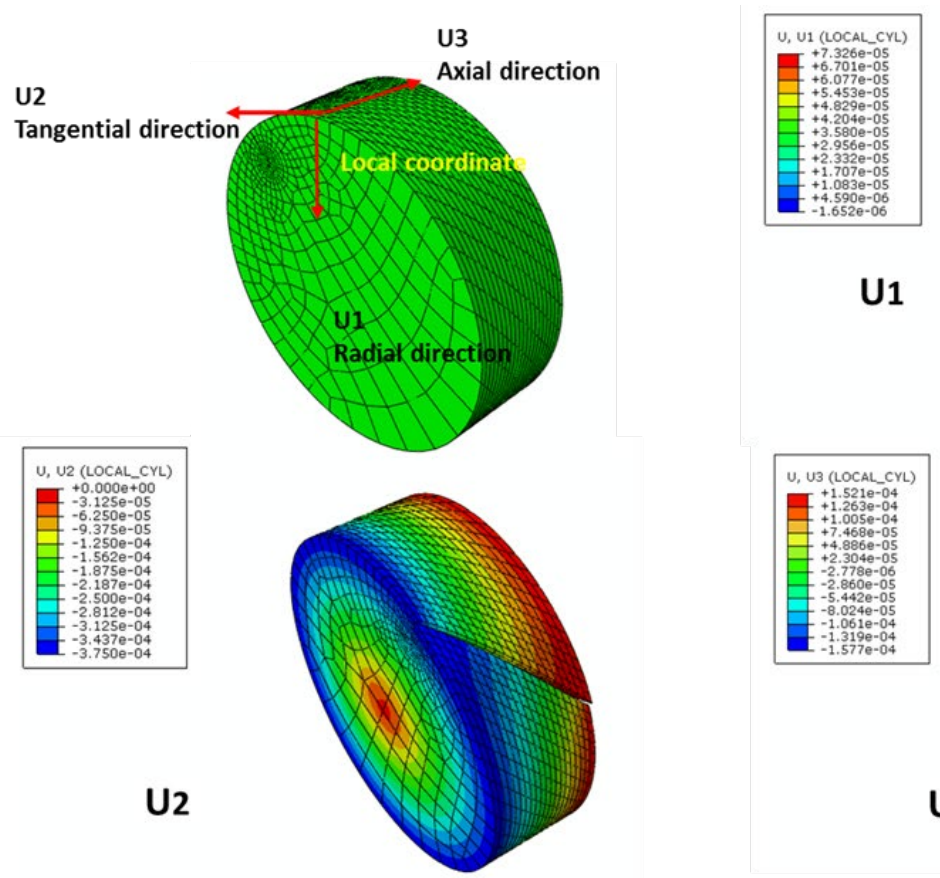

U1
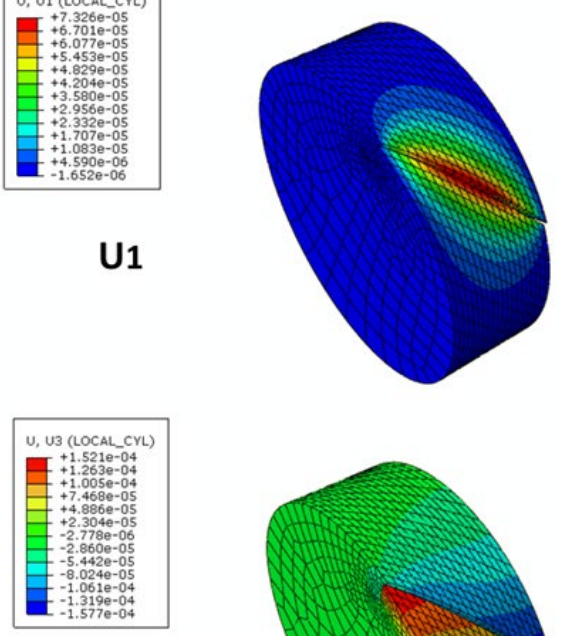

U3

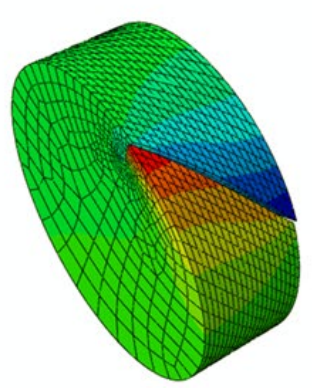

(b)

Fig. 6. (a) The evolution of crack growth in the SNTT sample with FEA simulation models. (b) Single crack depth example FEA displacement results, for $a / D=0.10, D=0.375$-inch, Steel, under end rotation theta $=0.002$ with ends fixed at $U 1$ and $U 2$ orientations. 
Fig. $7 \&$ Fig. 8 show the evolution of crack growth in an SNTT sample with $a / D$ ratio increases from 0.10 to 0.45 . Fig. 7a shows the compliance evolution of the SNTT sample with respect to different ratios of crack length over diameter. To obtain a more sensitive response of the compliance evolution, a factor was applied to account for the effect of unbroken ligament of the SNTT samples, which is graphed in Fig. $7 \mathrm{~b}$. It shows that the compliance evolution curves are the same for specimens with different materials or sizes. $\gamma$ is the unit end rotation angle; $T$ is the applied torque; $\mu$ is the shear modulus; and $R$ is the cylinder radius.

Fig. 8a shows the fracture resistance evolution of the SNTT sample with respect to crack length over diameter ratios. In order to obtain a more sensitive response of the compliance evolution, a factor also was applied to account for the effect of unbroken ligament of the SNTT samples, which is shown in Fig. 8 b. It was noted that fracture resistance curves are the same for specimens with different materials or sizes. $G$ is the energy release rate; $T$ is the applied torque; $\theta$ is the associated rotation angle; $A$ is the cross-section area of the cylinder.

\section{Unscaled SNTT Crack Growth Compliance Equation}

$\frac{\gamma}{T} \mu R^{4}=-0.6541\left(\frac{a}{D}\right)^{4}+0.5189\left(\frac{a}{D}\right)^{3}+1.7171\left(\frac{a}{D}\right)^{2}+0.2045\left(\frac{a}{D}\right)+0.6254$

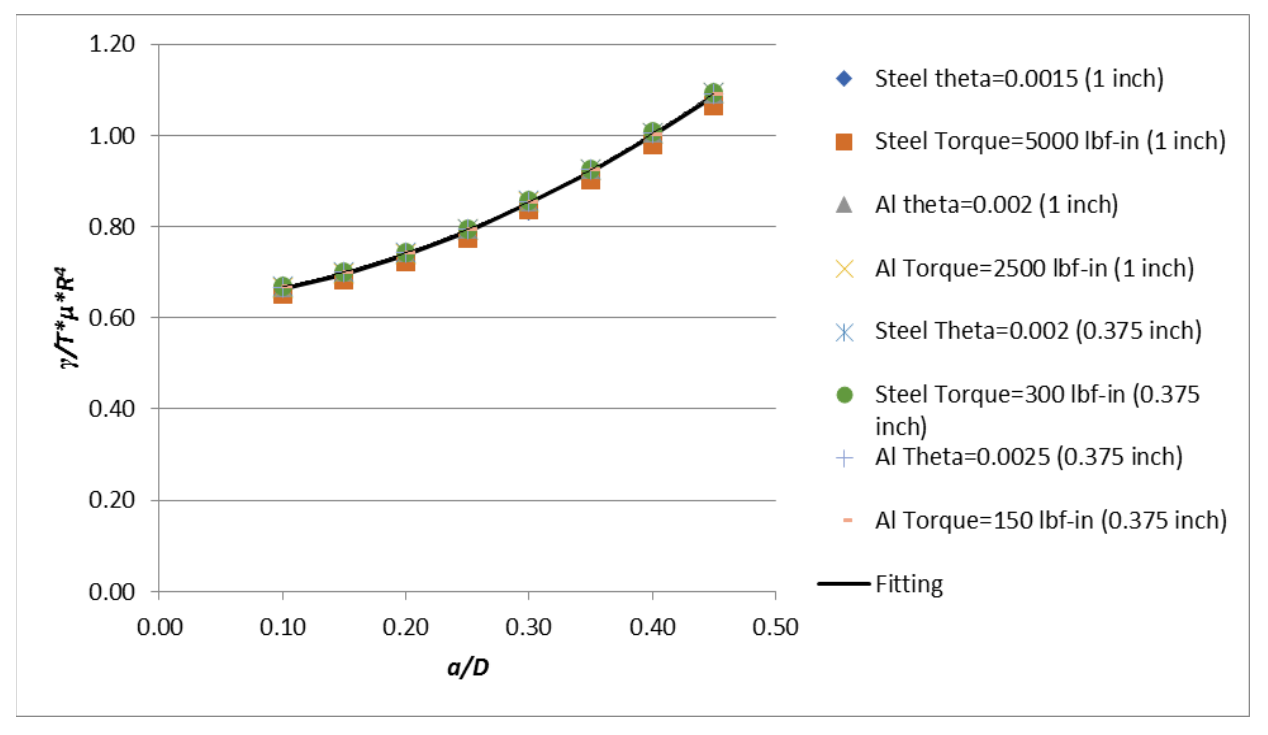

(a) 


\section{Scaled SNTT Crack Growth Compliance Equation}

$$
\frac{\gamma}{T} \mu R^{4}\left(1-\frac{a}{D}\right)^{4}=3.3445\left(\frac{a}{D}\right)^{4}-5.2514\left(\frac{a}{D}\right)^{3}+4.0568\left(\frac{a}{D}\right)^{2}-2.2298\left(\frac{a}{D}\right)+0.6226
$$

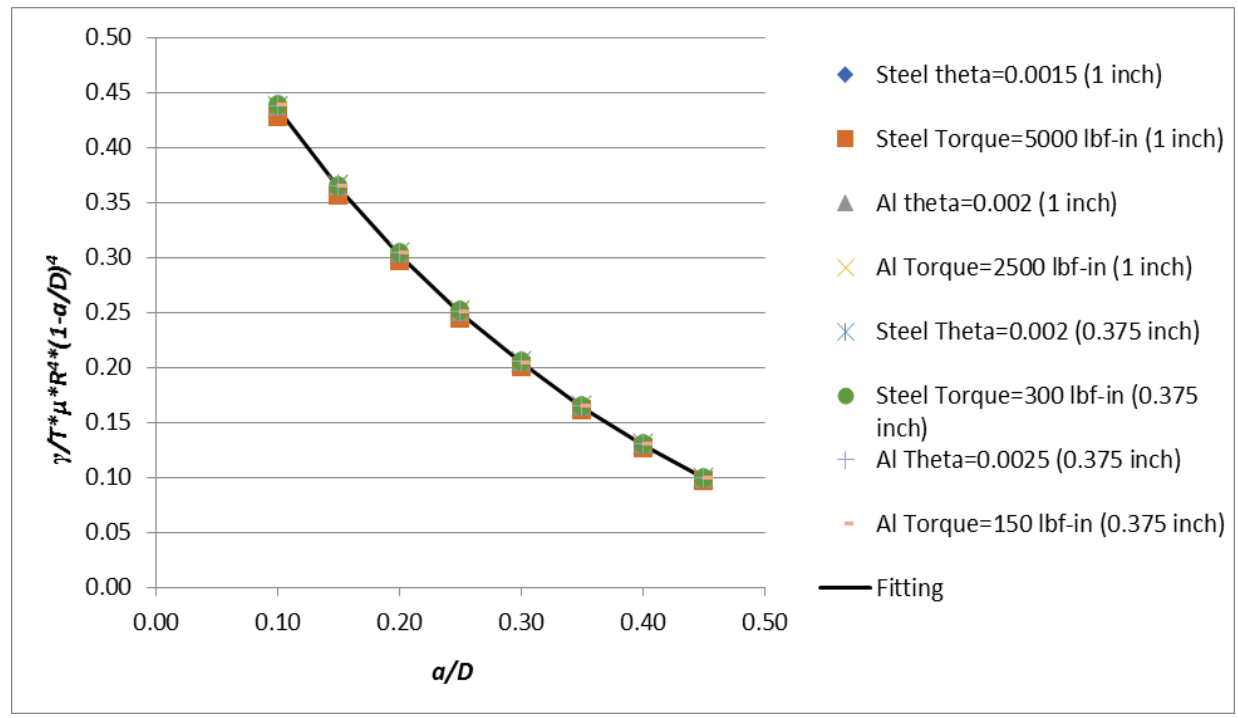

(b)

Fig. 7. (a) The unscaled compliance evolution with the crack growth; (b) The scaled compliance evolution along the crack growth with unbroken ligament factor.

\section{Unscaled SNTT Energy Release Rate Evolution Equation}

$$
\frac{G * A}{T * \theta}=-95.543\left(\frac{a}{D}\right)^{4}+107.14\left(\frac{a}{D}\right)^{3}-52.72\left(\frac{a}{D}\right)^{2}+16.028\left(\frac{a}{D}\right)-0.2489
$$

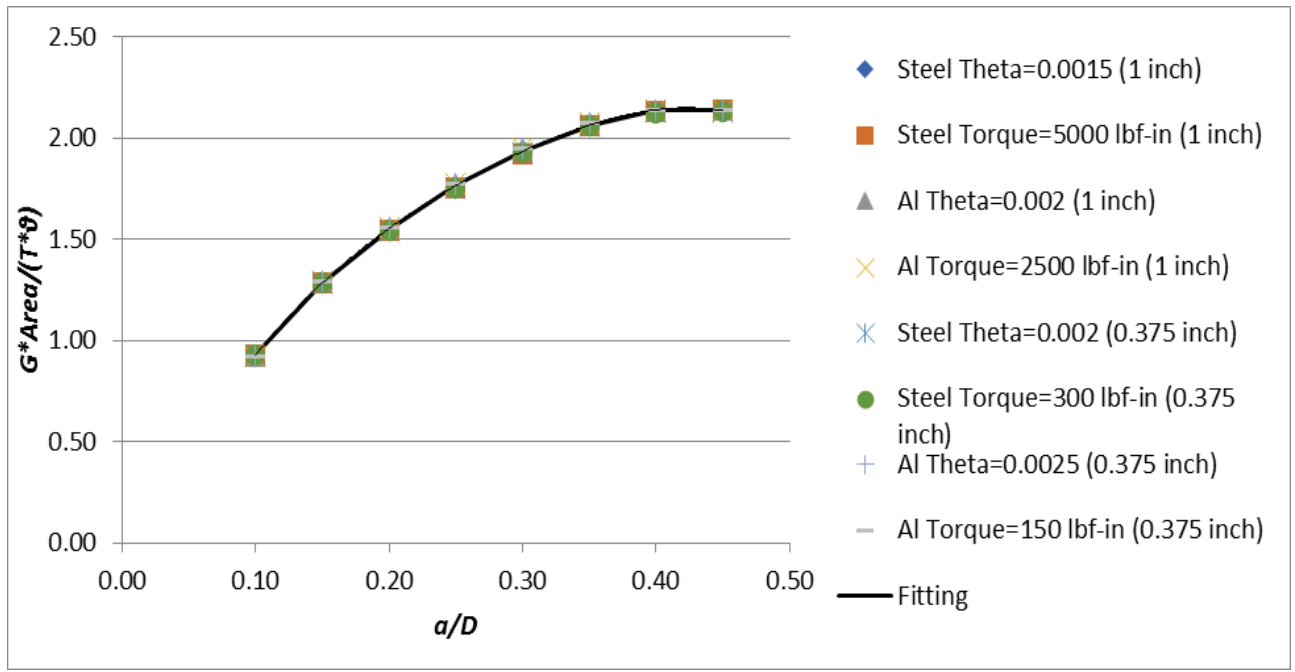

(a) 


\section{Scaled SNTT Energy Release Rate Evolution Equation}

$$
\frac{G * A}{T * \theta *\left(1-\frac{a}{D}\right)^{2}}=-154.56\left(\frac{a}{D}\right)^{4}+188.95\left(\frac{a}{D}\right)^{3}-62.398\left(\frac{a}{D}\right)^{2}+20.626\left(\frac{a}{D}\right)-0.4716
$$

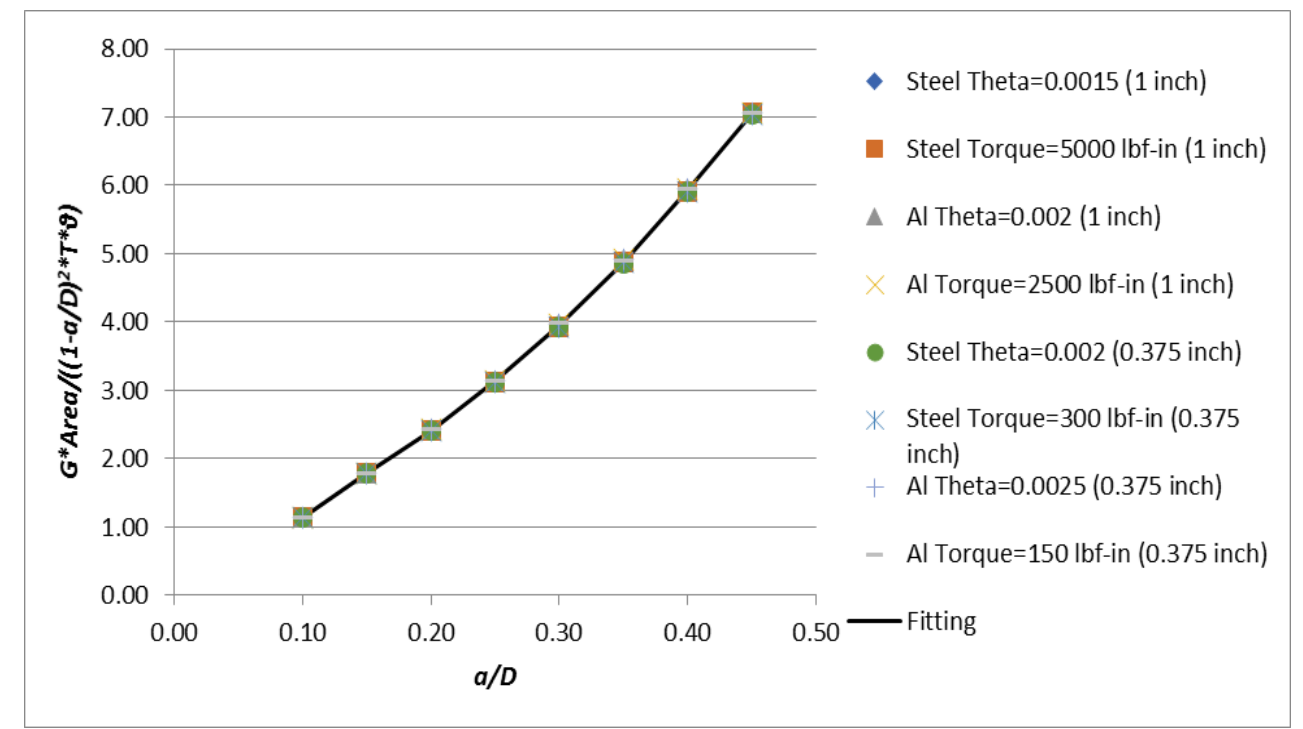

(b)

Fig. 8. (a) The unscaled energy release rate evolution with the crack growth; (b) The scaled energy release rate evolution along the crack growth with unbroken ligament factor.

\subsubsection{Experimental verification on the developed SNTT fatigue crack compliance protocol}

Experiment verification measurements were performed at different crack lengths during the cycle fatigue process. Fatigued specimens were cut in cross sections, and crack penetration depths were measured and compared with predictions from the developed SNTT compliance function. For the base material X52 pipe steel, a generally good agreement was observed between the crack length predictions and the postmortem experimental measurement. For the welded X52 materials, good agreements were also observed for most specimens. For some weld samples, crack deviation was observed during the cycle fatigue process. The reason for this deviation is mainly related to the flaws/impurities distribution of the welded material encountered in the fatigue pre-crack propagation path, based on postmortem examination. In order to validate the analytical models, further analysis was carried out on the rotary variable differential transformer (RVDT) measurement and finite element model predictions. The net section of the SNTT specimen was modeled with the measured crack depth. By using the torque data from the RVDT measurement, good agreement was observed between the predictions and the measurement. 
X52 weld SNTT samples were fabricated from a segment of friction stir welded X52 steel pipe. The thickness of the pipe is 0.5 inch and the diameter of SNTT samples was designed as 0.375 inch accordingly. The SNTT specimen axis was parallel to the pipe cylinder axis. One complete loop of spiral groove was machined on the X52 SNTT sample. The fractured X52 baseline SNTT sample is shown in Fig. 9a, the detailed fatigue pre-crack area is shown in Fig. 9b, and the cross-section of fractured X52 weld SNTT sample is illustrated in Fig. 9c.

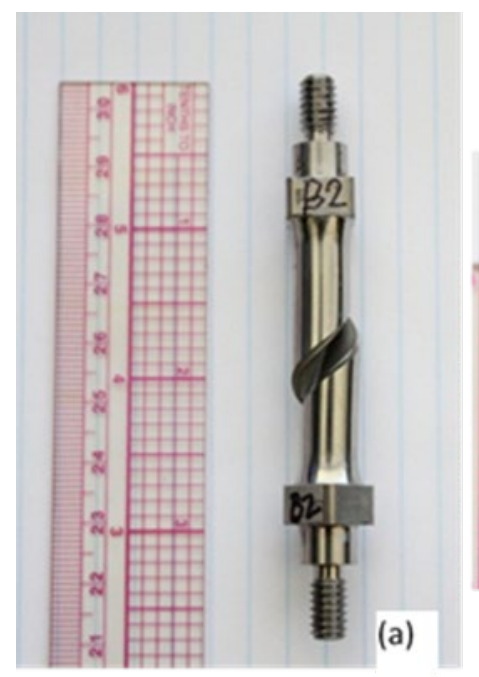

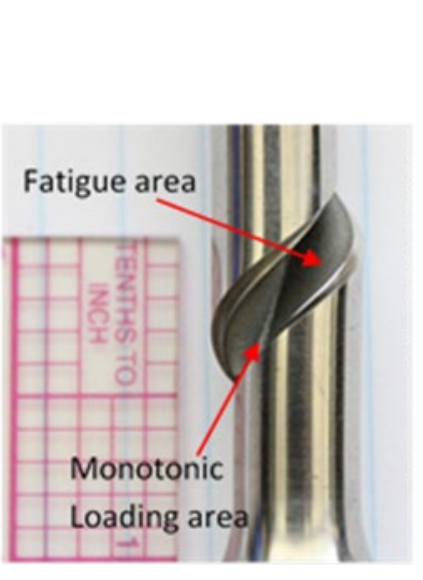

(b)

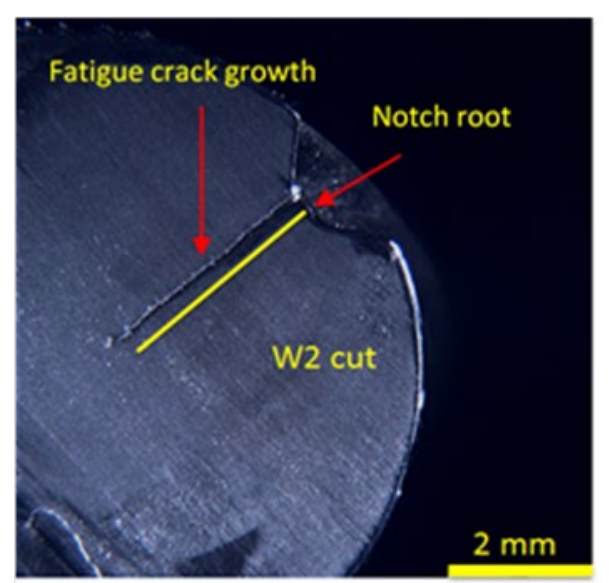

(c)

Fig. 9. The failed B2 specimen of X52 baseline sample, (a) entire view; (b) enlarged view of the middle section of tested SNTT sample, (c) the fatigue pre-crack growth profile of X52 weld SNTT sample W2, where the crack growth orientation is toward the central axis of the SNTT sample.

\subsubsection{J-Integral analysis development for solid rod SNTT specimens}

The J-integral represents a way to calculate the strain energy release rate, or work (energy) per unit fracture surface area, in a material [22,23]. In 1968 J. R. Rice published papers in which he discussed the potential of a path-independent integral, J, for characterizing fracture in non-linear-elastic materials. Rice defined the J-integral for a cracked body as follows:

$$
J=\int_{\Gamma}\left(W n_{1}-T_{i} \frac{\partial u_{i}}{\partial x_{1}}\right) d s
$$

where $W$ is the strain energy density function given by $W=\int \sigma_{\mathrm{ij}} \mathrm{d} \varepsilon_{\mathrm{ij}}, T_{\mathrm{i}}$ is the traction vector defined as $T_{\mathrm{i}}$ $=\sigma_{\mathrm{ij}} \mathrm{n}_{\mathrm{j}}, \sigma$ is the stress tensor, $\mathrm{u}$ is the displacement vector, $\Gamma$ is a contour surrounding the crack tip in the anticlockwise direction from the lower face to the upper face of the crack. $n_{j}$ are direction cosines of the 
outward normal on $\Gamma$, and $\mathrm{d} s$ is an infinitesimal arc length along $\Gamma$.

The $J$-integral has been shown to be equivalent to the rate of release of the potential energy $\mathrm{U}$ with respect to the crack extension [22]: for a body of thickness B,

$$
J=-\frac{1}{B} \frac{\partial U}{\partial a}
$$

Where, $a$ represents crack length, and

$$
U=\int_{A} W(x, y) d A-\int_{S_{T}} T_{i} u_{i} d s
$$

represents the system potential energy. Where $A=$ area of the body and $T_{i}$ are $u_{i}$ are the tractions and displacement, respectively, applied along the boundary $\Gamma$.

An alternate and equivalent definition of $\mathrm{J}$ can be written as follow [24],

$$
J=\frac{1}{B} \int_{0}^{\Delta}\left(-\frac{\partial P}{\partial a}\right)_{\Delta} d \Delta
$$

or,

$$
J=\frac{1}{B} \int_{0}^{P}\left(\frac{\partial \Delta}{\partial a}\right)_{P} d P
$$

These representations relate $\mathrm{J}$ to the rate of change with respect to crack size, a, of the area under the load versus load-point-displacement, $\mathrm{P}$ versus $\Delta$, curves. Here $\mathrm{P}$ is the force and the curves are generated for different crack sizes, a, where specimens subjected to monotonic loading. This definition was used by Begley and Landes in the experimental evaluation of the J versus $\Delta$ relation [6].

\subsubsection{J-Integral for a deep spiral notch crack SNTT specimen under pure torsion}

Rice first recognized that for configuration with a single characteristic dimension dominating the deformation behavior such as uncracked ligament, $b=W-a$ [24]. For a deeply cracked SNTT specimen, the relative additional angle of the grip end rotation due to the presence of the crack, $\theta_{c}$, can be estimate from a dimensional analysis. Consider a SNTT rod specimen with diameter, $D$, subjected to a pure torsion force, $T_{q}$, which is transmitted through a narrow neck of width, $b=D-a$, between spiral crack front and the cylinder free surface edge. For an applied torque $T_{q}$ of SNTT configuration with the spiral crack front length, $\mathrm{B}$, the $\theta_{c}$ can be expressed as,

$$
\theta_{c}=f\left(\frac{T_{q}}{B b}\right)
$$


The torque end rotation angle, $\theta_{\text {total }}$, can be regarded as the sum of the rotation angle without cracks, $\theta_{n c}$, plus the rotation angle due to the introducing the crack, $\theta_{c}$, i.e., $\theta_{\text {total }}=\theta_{n c}+\theta_{c}$. And because $\theta_{n c}$ does not dependent on crack length, $a$, then

$$
\frac{\partial \theta_{\text {total }}}{\partial a}=\frac{\partial \theta_{c}}{\partial a}
$$

Thus, J can be written as:

$$
\begin{aligned}
& J=\frac{1}{B} \int_{0}^{T_{q}}\left(\frac{\partial \theta_{c}}{\partial a}\right)_{T_{q}} d T_{q}, \\
& \left(\frac{\partial \theta_{c}}{\partial a}\right)_{T_{q}}=\left(\frac{\partial \theta_{\text {total }}}{\partial a}\right)_{T_{q}}=-\left(\frac{\partial \theta_{\text {total }}}{\partial b}\right)_{T_{q}}=-\left(\frac{\partial \theta_{c}}{\partial b}\right)_{T_{q}}
\end{aligned}
$$

Replace $\theta_{c}$ with $f\left(T_{q} / B b\right)$, and $J$ can be written as:

$$
J=-\frac{1}{B} \int_{0}^{T_{q}}\left(-\frac{T_{q}}{B b^{2}}\right) f^{\prime}\left(\frac{T_{q}}{B b}\right) d T_{q}
$$

where

$$
\begin{gathered}
f^{\prime}=d f / d\left(\frac{T_{q}}{B b}\right) \\
d \theta_{c}=f^{\prime}\left(\frac{T_{q}}{B b}\right) \cdot \frac{d T_{q}}{B b}
\end{gathered}
$$

Hence,

$$
J=\frac{1}{B} \int_{0}^{\theta_{c}} \frac{T_{q}}{b} d \theta_{c}=\frac{1}{B b} \int_{0}^{\theta_{c}} T_{q} d \theta_{c}
$$

where, $\int_{0}^{\theta_{c}} T_{q} d \theta_{c}$ is the area under the $T_{q}$ - $\theta_{c}$ curve.

The alternative in implementing equation above is stated as following:

The full angle $\theta_{\text {total }}$ is usually measured. However, for a deeply cracked specimen, $\theta_{n c}$ is small and we can usually assume that $\theta_{\text {total }} \cong \theta_{c}$. Thus, $J$ integral for SNTT approach can also be written as:

$$
J=\frac{1}{B b} \int_{0}^{\theta_{\text {total }}} T_{q} d \theta_{\text {total }}
$$




\subsection{Typical SNTT experimental procedures and results}

SNTT tests were performed on a biaxial testing system shown in Fig. 1. Shear strain was measured using a biaxial strain extensometer and/or a Rosette strain gage for cross calibration; and the torque-ends rotation angle was measured by RVDT. Pure torsion was achieved with a zero axial force in control. Precracking for metallic specimens was accomplished with cyclic torsion using Haver sine wave form. The maximum torque used in pre-cracking varies with materials and must be determined experimentally. In the case of an A302B steel sample with 1.9-mm deep notch, $60-80 \%$ of the torque that generates the maximum allowable shear stress around the specimen surface will suffice. The A302B specimen (Fig. 10) has a uniform gage section of 20.3-mm diameter and 76.2-mm gauge length. A A302B specimen having a spiral V-groove with a depth of 1.9-mm was tested. The specimen (Fig. 10) fractured at $519.7 \mathrm{~N}-\mathrm{m}$ torque at room temperature. Test results obtained from the strain gage and biaxial extensometer are shown in Fig. 11a \& Fig. 11b, respectively [12].

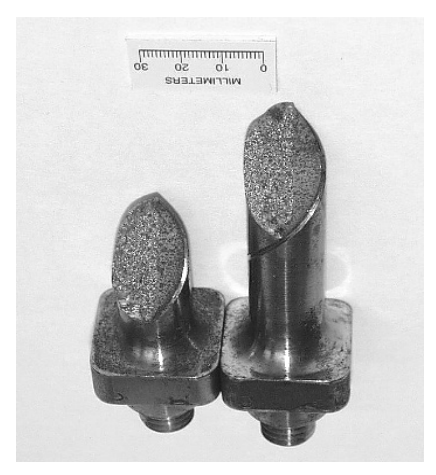

(a) front view

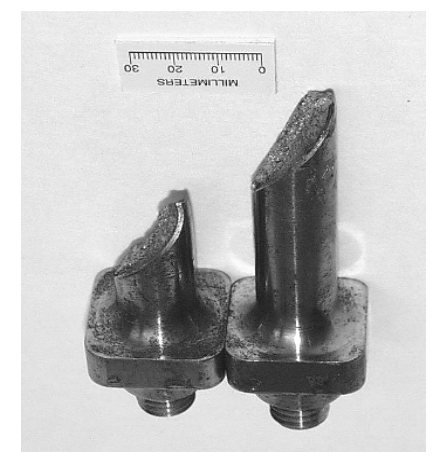

(b) side view

Fig. 10. The fractured profiles of tested SNTT A302B specimen.

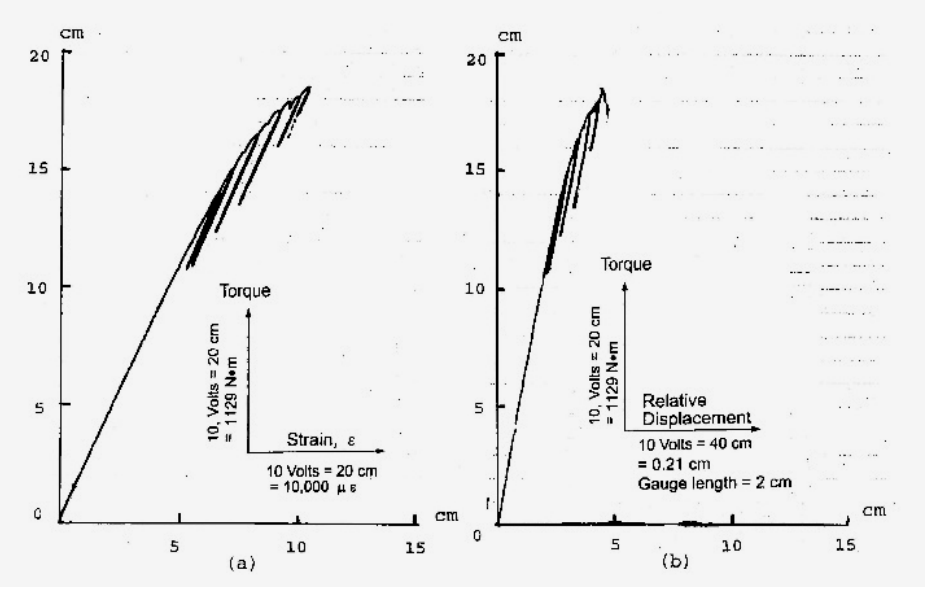

Fig. 11. Plot of SNTT test results from (a) load cell and strain gauge, and (b) load cell and biaxial extensometer. 


\section{BIAXIAL TESTER DEVELOPMENT TO PERFORM SNTT TESTING PROTOCOL}

\subsection{Biaxial tester development background}

The biaxial tester will be used for fracture toughness testing using ORNL SNTT methodology in assisting DOE Used Fuel Disposition campaign (UFDC) program. There are two specific programs will be using the information gained from fracture toughness determination of the targeted structural components, namely, UFDC SNF dry storage canister program development, and UFDC Sister Rods program development in supporting SNF interim storage and transport related initiative. The outcome of this project will further ensure safe operation of US nuclear power initiative during back end nuclear fuel cycle. The device will eventually be resided in a nuclear fuel hot-cell facility; the baseline product of biaxial tester needs further modification to meet hot-cell operation specifications. Due to physically located in such high irradiation environment, the hydraulic fluid is not preferred in such environment. Thus, electrical-magnetic force driver became the viable choice for fuel hot-cell operation. An Axial/Torsion electro-dynamic test machine was developed by TestResource to carry out SNTT testing for fracture toughness evaluation, the associated user manual is provided in APPENDIX A.

Electro Dynamic Test Technology offers advantages over traditional servo hydraulic test technology:

(1) More reliable with low maintenance, low audible noise and lower purchase price than hydraulic technology.

(2) Improved 24 bit machine control makes it possible to control sub gram level loads and submicron positions.

(3) Modular approach - makes it possible to swap elements and configure to specific test goals (speeds, loads, strokes, channels). The 830L can be user re-configured as a single biaxial station or two separate axial and torsion test stations. Modularity makes customization affordable.

\subsection{Biaxial tester design specifications}

The design specifications of the developed biaxial tester are the followings:

$\underline{830 \text { Axial Torsion Machine }}$

* 5,500 lbs / 2500 in-lbs Capacity

* Configured with Torsion on Top / Axial mounted on bottom

830-48w20 Inverted Axial Torsional Dual Column Load Frame

* Floorstanding 
* 20" (508 mm) clearance between columns

* 42" (1065 mm) daylight or crosshead adjustment

* Torsion actuator in top fixed crosshead

* Axial actuator in adjustable bottom crosshead

* With provision to attach Load cell on Axial Actuator

\section{EF5210-3S Electro Dynamic Actuator}

* Static \& Fatigue load rating: \pm 24 kN (5500 lb)

* Max speed: $200 \mathrm{~mm} / \mathrm{s}(8 \mathrm{in} / \mathrm{s})$

* Max frequency: $15 \mathrm{hz}$

* Stroke: $\pm 125 \mathrm{~mm}( \pm 5$ in $)$

* Antirotate Fixture

* Encoder 0.2 micron resolution

$\underline{\text { TYD200 Torsional Actuator }}$

* Static rated $200 \mathrm{Nm}$ (1770 in-lb)

* Dynamic rated $281 \mathrm{Nm}$ (2493 in-lb)

* Max speed $1500 \mathrm{deg} / \mathrm{sec}$

* Encoder resolution $\sim 0.2$ arc min

* Backlash free

Axial Torsion Power Pack

* 400V 3 phase power

* 10 foot long cables

\section{$\underline{\text { FAT 10K/6K Fatigue Rated Axial Torsional Load Cell }}$}

* Measures load and torque with minimal crosstalk

* Fatigue rating: 10,000 lb (44 kN) Axial

* Fatigue rating: 6000 in $\mathrm{lb}(660 \mathrm{Nm})$ Torsional

* Cable with connectors 


\section{High Performance Servocontroller}

* 40 bit Servo loop update

* Data acquisition up to $6 \mathrm{kHz}$

* Position Encoder, Load, and Strain signal conditioners with 24 bit analog data conversion

* Additional Channels, Position Encoder, Strain, 2 Hi Level (+/-10V)

* Autocalibration on all strain channels

* 8 Ch digital output (control bits) \& 8 Ch digital input (sense bits)

* 2 - 16 bit Servo/ Daq Output

* Ethernet interface

237032 Bit Test Software Products

* Global Data Sharing (GDS) Compatibility - enables developers to extend and add test modules

* MTL32 MachineBuilder Software

- create multiple machine setups,

- real time adjustable servo tuning,

- calibration of each channel

* TestBuilder Software

- Fatigue, static, and multi-step programming

- Stroke, load and strain control with mode switching

- Meters show current, max-min, peak-valley readouts, command output, and cycle counters

- Displays include multiple real time scopes,

- Data recording is time and peak-valley based. Export data to MS Excel and txt file formats.

- Data reduction options for real time data logging and during data exporting

Computer for Dynamic Controllers

* i5 - Processor (3.2 GHz min)

* Win7 Professional (x64) or later

* 8 GB RAM Minimum 
* 500 GB HD Minimum

* Integrated Video card

* Onboard 2.0 USB \& 1GB Networking Interface

* 22" Flat Panel Monitor Minimum

* Integrated Networking Card \& Networking Cables as Needed

* Keyboard \& Mouse

The design configurations overview are shown in Fig. 12, where ORNL site requirements for the power sources are

1. 230 VAC $3 \mathrm{PH}, 35 \mathrm{~A}, 50 / 60 \mathrm{~Hz}$ - Main system power

2. (X3) 110-240 VAC 1 PH - Standard outlets for PC, monitor, and controller power.

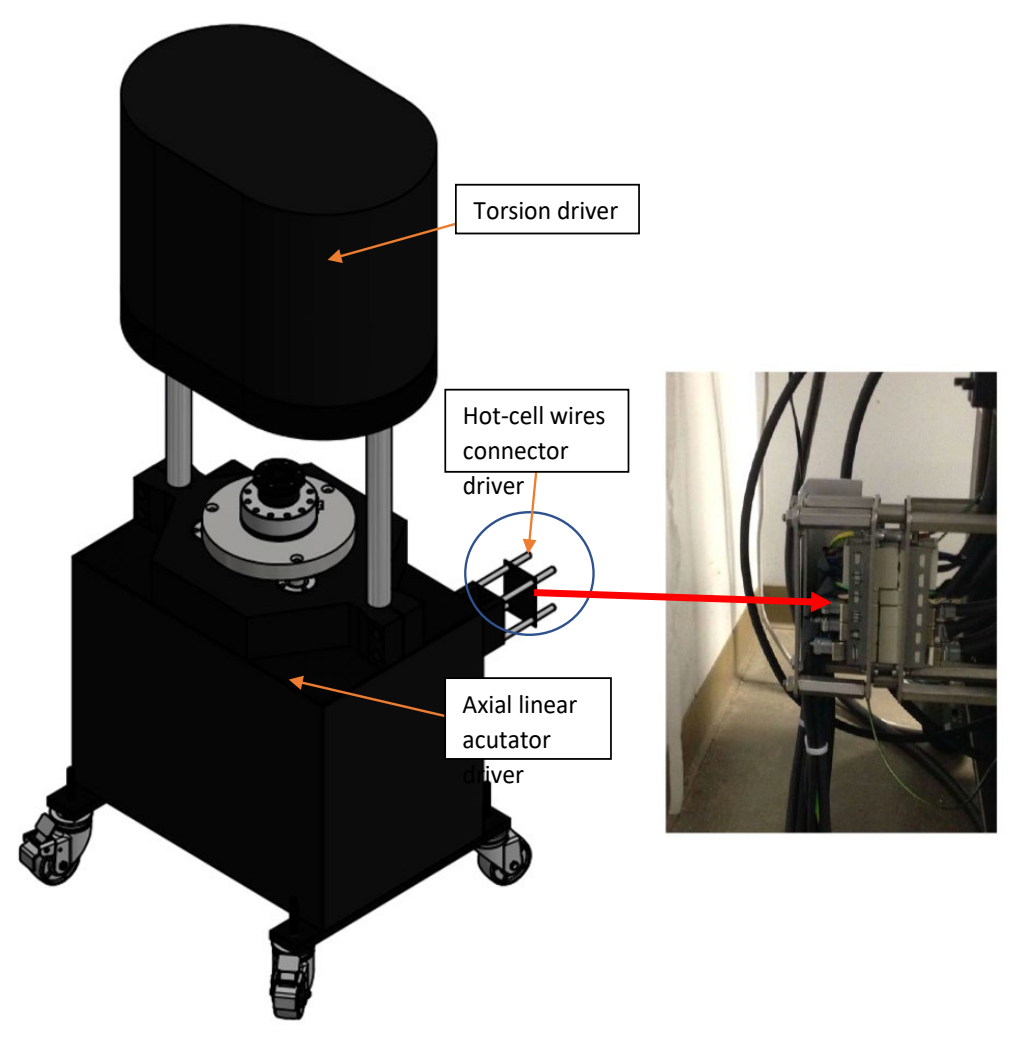

(a) Axial/Torsion biaxial tester schematic diagram. 

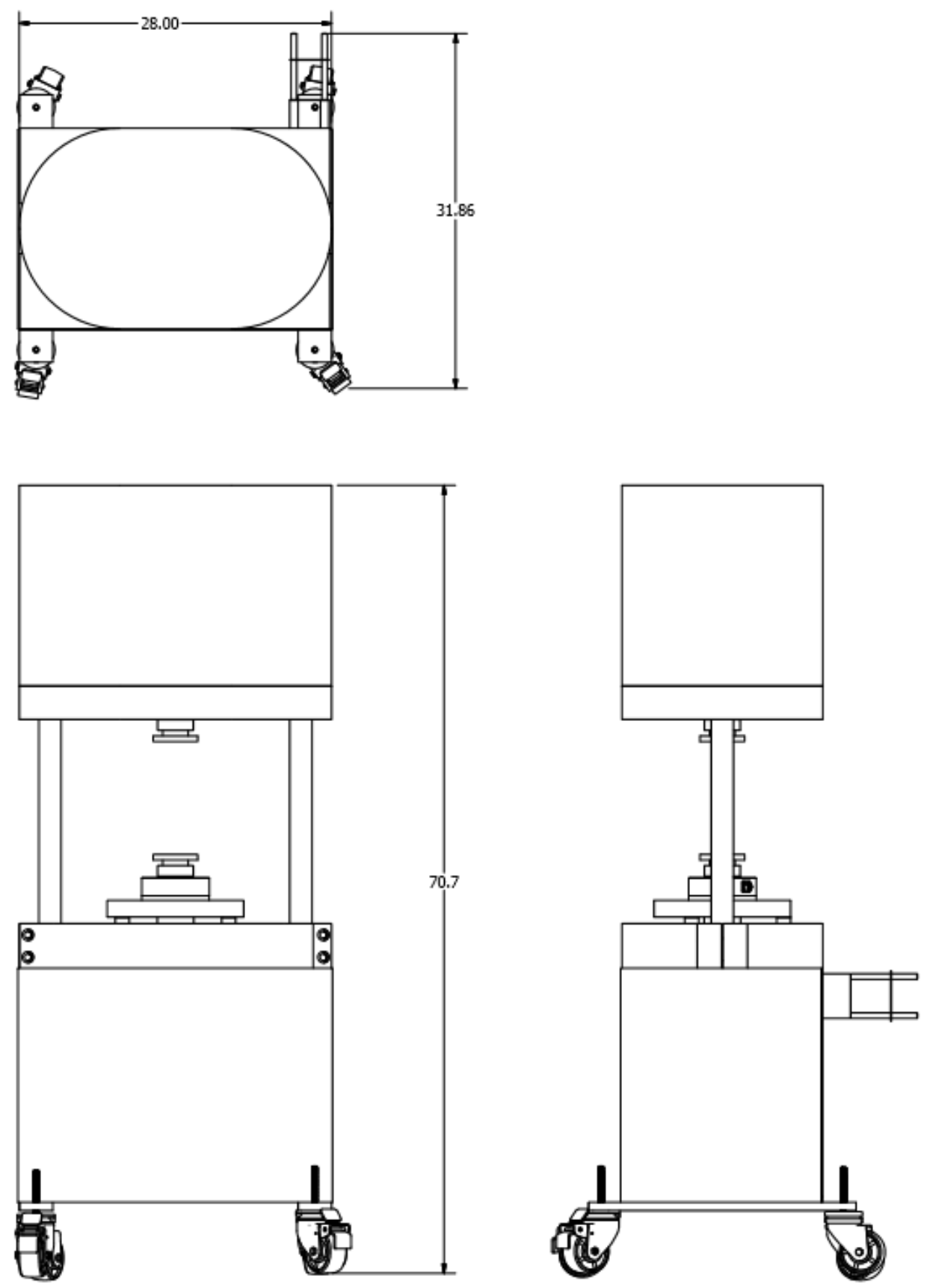

(b) Top crosshead fully lowered - units inches 

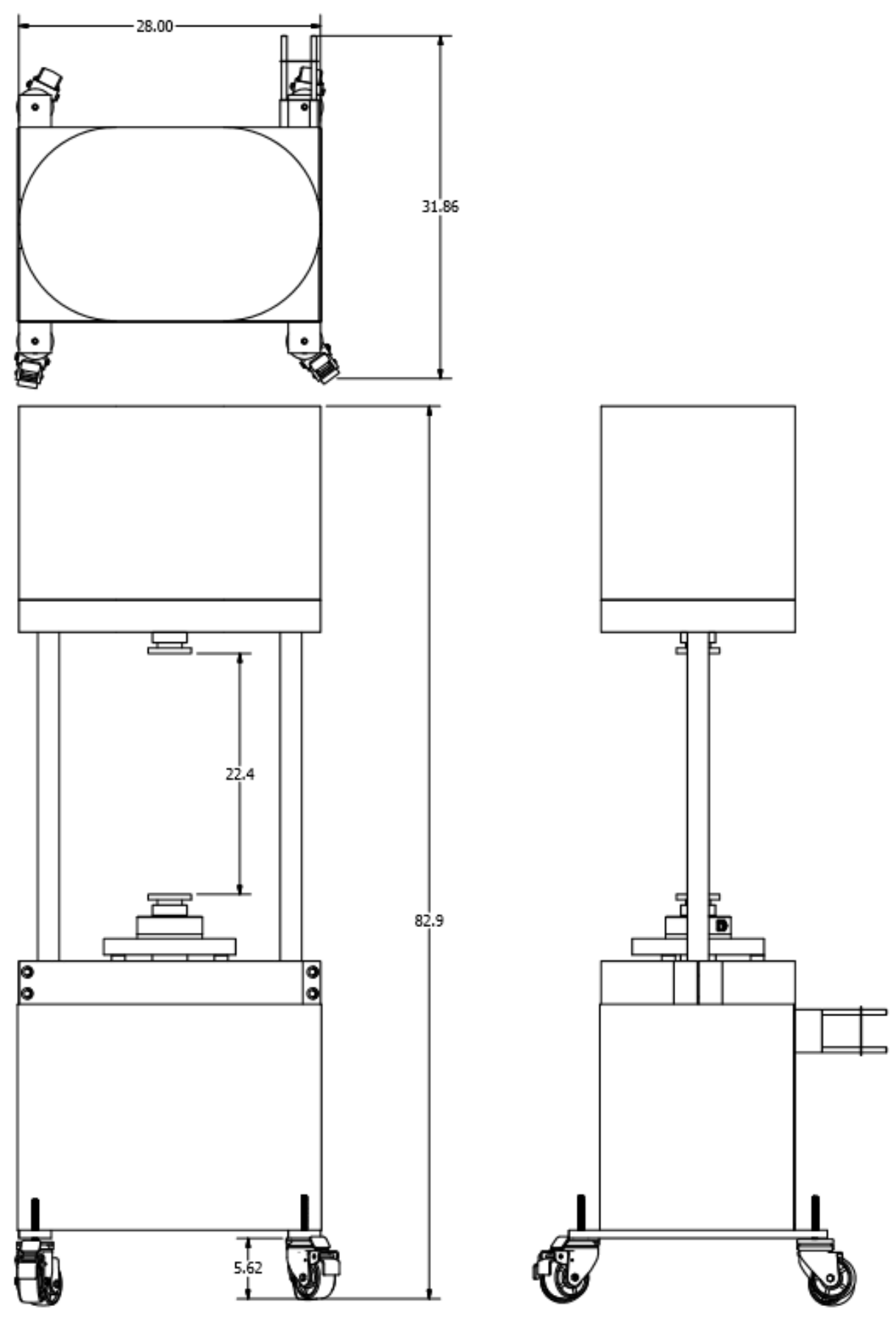

(c) Top crosshead fully extended - units inches 

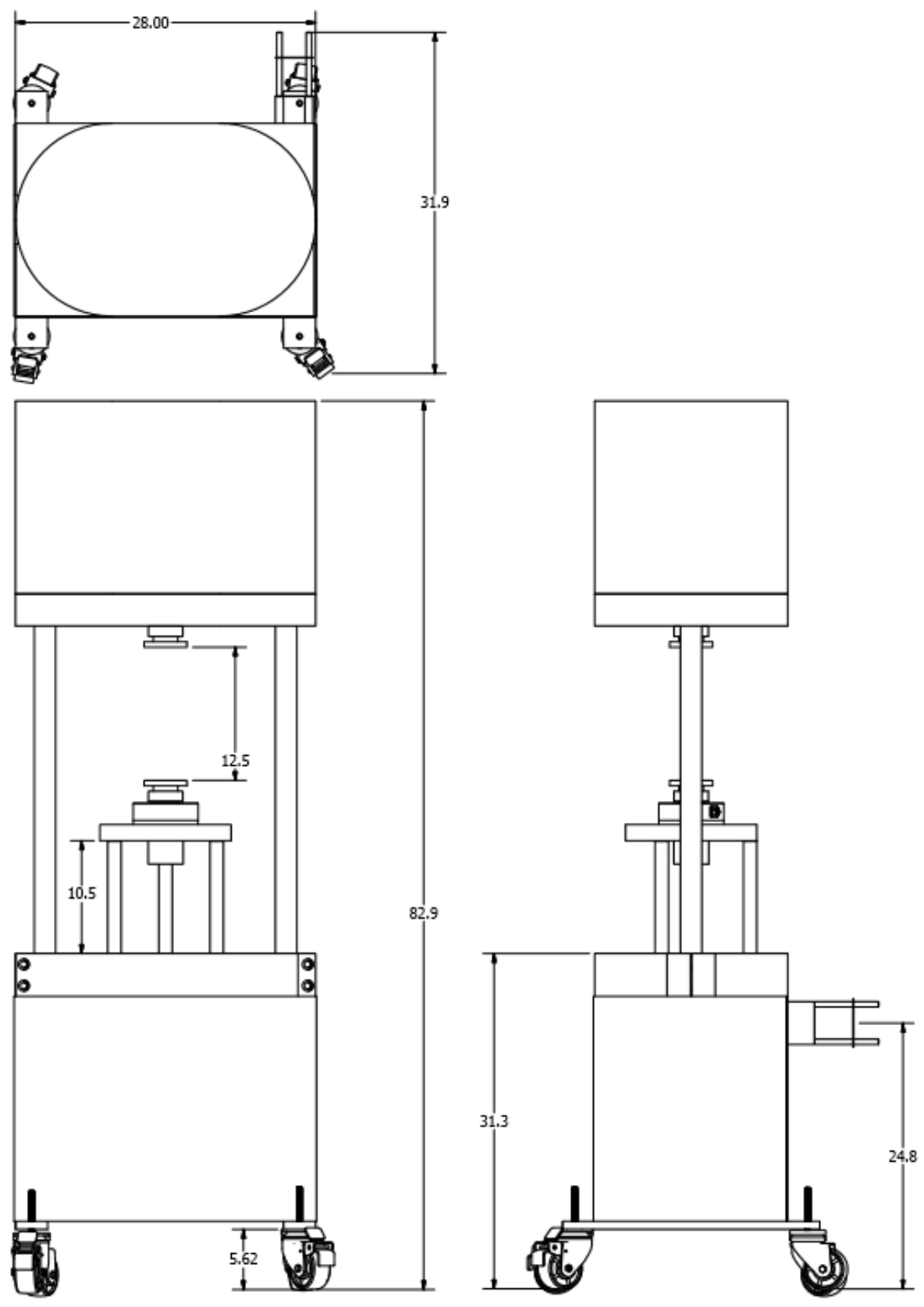

(d) Top crosshead fully extended, and actuator fully extended

Fig. 


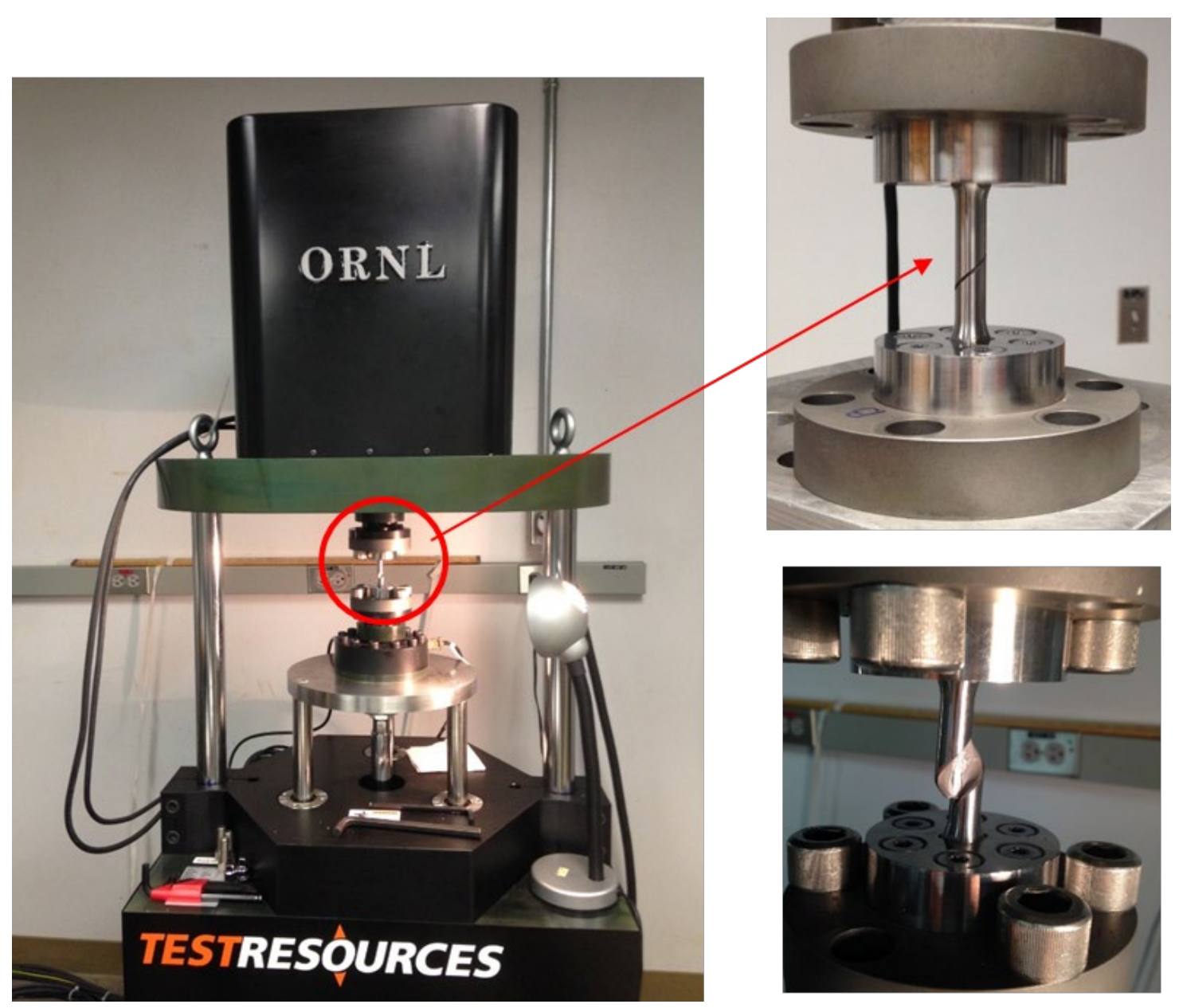

(e) Biaxial tester with sample end grips, including tested SNTT fatigue fracture sample

Fig. 12. TestResource Axial/Torsion biaxial tester design configurations. 


\subsection{Biaxial tester hot-cell implementation readiness}

The additional tasks proposed for implementing the developed biaxial tester in the hot-cell environments to carry out SNTT testing are detailed below.

Task 1: Develop the sample end-grip system for holding and loading the SNTT sample into the biaxial tester in a hot-cell operation. The Task 1.1,1.2, and 1.3 will be intertwined during program development; and may require several iterations to complete the tasks.

Task 1.1: Design sample end-grip system for the SNF rod to form the SNTT sample, which considers the followings:

(1) Grip system can provide sufficient grip to hold the SNF rod at ends, where epoxy molding protocol and the associated bolt tightening mechanism to secure the SNF rod firmly into the grip rigid-sleeve system will be deigned,

(2) The designed grip system that integrated with SNF rod can transmit sufficient shear force from biaxial tester loading train to the SNF rod system to enable performing the cyclic fatigue testing and the final fracture testing on the tested SNF rod, without the potential of damaging the SNF rod at SNF rod and grip-ends system contact interfaces,

Task 1.2: Develop SNTT sample in-cell casting mold that can anchor the SNF rod into the designed end-grip system.

This device will provide the epoxy molding functionality, and the screw bolting system to tighten the SNF rod after epoxy curing. This combined epoxy molding and screw tightening mechanism can effectively increase the contact pressure at SNF rod and end-grip interface, i.e., increase the interface bonding strength, to securely anchor the SNF rod into end-grip holder system for the follow-on fatigue and fracture testing.

Task 1.3: Develop automated biaxial tester system operational protocol for installing the SNTT sample into biaxial tester loading train, which will consider the followings:

(1) The modified end-grip system geometry combined with the to-be developed tester system control operation procedure will provide an effective means for mounting SNTT sample system, equipped with self-align functionality, into biaxial tester loading train. A safe installation operation protocol with pre-defined operational displacement and force limits for SNF rod protection will be developed to provide automated sample installation procedure in a hot-cell. 
(2) This is one of the essential operations to enable the SNTT testing in a hot cell, environment due to inability of normal hand-on operation for SNTT sample installation performed at an out-of-cell environment. The criteria of such protocol development need to consider an easy installation of SNTT sample in the hot-cell and prevent the potential SNF rod damage occurred during SNTT sample installation or mounting. This is because that the potential high compression force can arise to the SNF rod system upon engaging SNTT rod system into the torsion tester loading train adaptor system under uplifting a SNTT sample. Another important consideration is the protocol should have multiple or repeatable operation functionality carried out automatically, which allows re-installation upon the failure of the initial attempts to load the test sample into tester loading train adapter.

Task 2: Develop SNTT experimental testing and fracture toughness analytical evaluation procedures for tubing structure materials fracture behavior evaluation. The associated subtaks are stated below; Task 2.1, 2.2, and 2.3 are intertwined and may require several iterations to complete the tasks.

Task 2.1: Develop and quantify the SNTT test sample dimensions requirement that is suitable for SNF rod cladding fracture testing study; and prepare the surrogate SNTT samples for the out-of-cell SNTT tubing structure materials pilot testing.

The newly developed end-grips will be integrated with the surrogate rod to perform the reliability and integrity study of the SNTT sample system interface bonging strength to further quantify the defined interface surface area sufficiency of transmitting sufficient torsion load to the SNF rod without damage the SNF rod at interface regions.

Task 2.2: Develop experimental testing protocols for the cyclic fatigue and final fracture of SNTT rod sample.

Develop cyclic fatigue testing protocol with the associated initial flaws profile, in the category of through thickness crack or surface shallow spiral notch crack align with the principle stress profiles, to determine the target crack length and to estimate the needed cyclic loading intensity to induce such crack growth.

Develop systematic testing protocol to evaluate the cladding and insert pellet interface bonding efficiency through torsional cyclic fatigue testing, where the cut-off fatigue cycle \# that can trigger the sufficient drop of the interface bonding efficiency will be investigated. Develop the final fracture testing protocol at the designated fatigue pre-crack length that can 
enable the final fracture of the tested cladding sample. The estimate fracture torque will then be used to estimate the cladding material fracture resistance capacity, or fracture toughness property.

Both load control and displacement control approaches will be investigated for the cyclic fatigue and final fracture testing protocols developments.

Task 2.3: Develop the analytical evaluation protocol to determine the fracture toughness of the cladding structure materials, which cover the followings.

(1) The primary objective is to develop the close-form governing equations that can be used for fracture toughness evaluation, utilizing SNTT approach on tubing structure materials. Where a literature survey on the current approach for tubing structure materials fracture toughness evaluation will be carried out. The pro and con of the conventional approaches and SNTT approach for the SNF clad toughness evaluation will be evaluated.

The analytical approach development will be focused on the through thickness crack growth fracture profile scenario, which is an effective means to evaluation the associated clad fracture toughness. This type through thickness crack growth in a thin shell clad structure failure mode is a typical clad failure mode; for instance, it was also observed from (through-thickness) axial crack growth fracture of fuel cladding from an RIA test, or from a pressurized water piping failure.

The sensitivity study on the "total crack length" variation to the clad system fracture resistance capacity will be performed and quantified through both the experimental and analytical approaches. This sensitivity study can provide the optimized "total crack length" that can effectively trigger the final fracture of the tested clad sample; thus, SNTT sample with such fatigue pre-crack length can be used to accurately estimate a valid fracture toughness for the cladding tubing structure materials. This crack length selection related subject is also strongly dependent on the brittle or ductile nature of the targeted cladding materials.

If no analytical close-form solution for fracture toughness evaluation can be developed, FEA approach will then be used to develop numerical and analytical integrated protocol for fracture toughness or energy release rate evaluation of tested samples. 
(2) The cladding crack initiation site sensitivity study will also be carried out using FEA modeling approach. Where the crack growth potential initiated at ID and OD of the cladding tubing structure under torsion loading will be investigated. Which will provide additional guidance for carrying out the cyclic fatigue testing on SNTT cladding sample to develop sufficient total crack length (with through thickness crack profile). This sensitivity study can be used to estimate the incubation period of a surface crack becoming a through thickness crack. Combined with the typical through thickness crack growth rate obtained from fatigue testing protocol of Task 2 and surface crack growth rate estimate form sensitivity study, one can then effectively to carry out the cyclic fatigue testing protocol on the SNF rod to develop the SNTT sample with the targeted "total fatigue per-crack length," for final fracture test to evaluate the associated fracture toughness of tested cladding materials. 


\section{FRACTURE TOUGHNESS EVALUATION FOR SNF DRY STORAGE CANISTER WELD}

To meet the need for additional data on the dry storage canister material properties, the DOE Used Fuel Disposition campaign program has procured a full-diameter cylindrical mockup of a dual certified 304/304L SS storage canister produced using the same manufacturing procedures as fielded SNF fuel interim storage canisters. ORNL recently received five such weld plates, three longitudinal welds and two circumferential welds.

\subsection{SNTT Specimens Designs and Configurations}

In this proposed approach, SNTT samples were fabricated from several as-received Sandia SS 304/308 weld plates. Since the thickness of the weldment is $5 / 8$ inch, the diameter of the SNTT cylinder was designed to be 0.375 inch (Fig. 13). The SNTT specimen axis was parallel or perpendicular to the canister cylinder axis, pending on the received weldment is axial or circumferential weld. There are several types of spiral grooves designs with different notch depths, as illustrated in Fig. 14. The details of weld and HAZ SNTT specimen location machined from the weldments are illustrated in Fig. 15 and Fig. 16, respectively. Weld specimen only one single loop was machined in the SS304/308 weld SNTT sample, where for the deep notch HAZ samples two loops spiral groove was designed. Threads were introduced onto both ends of the SNTT samples.
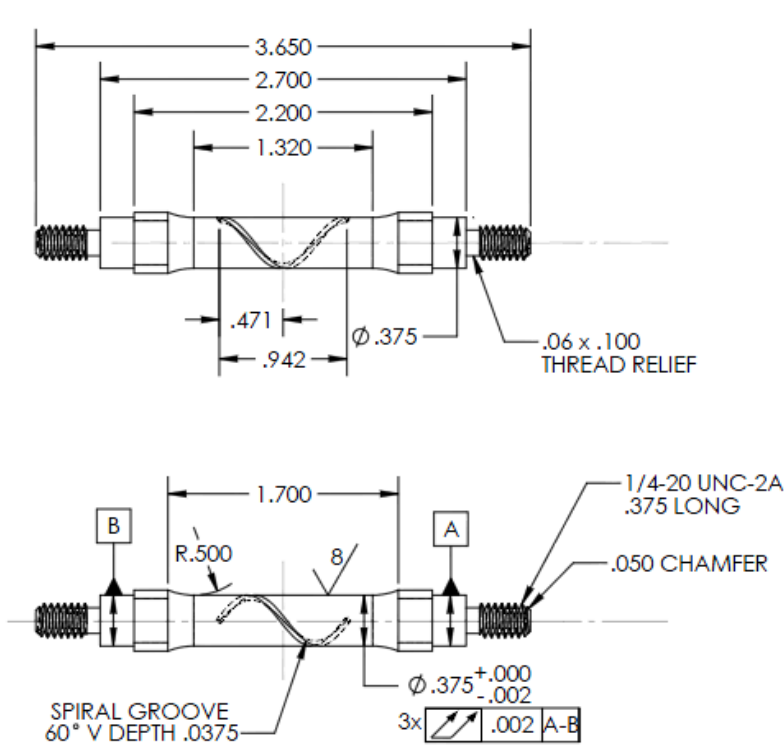
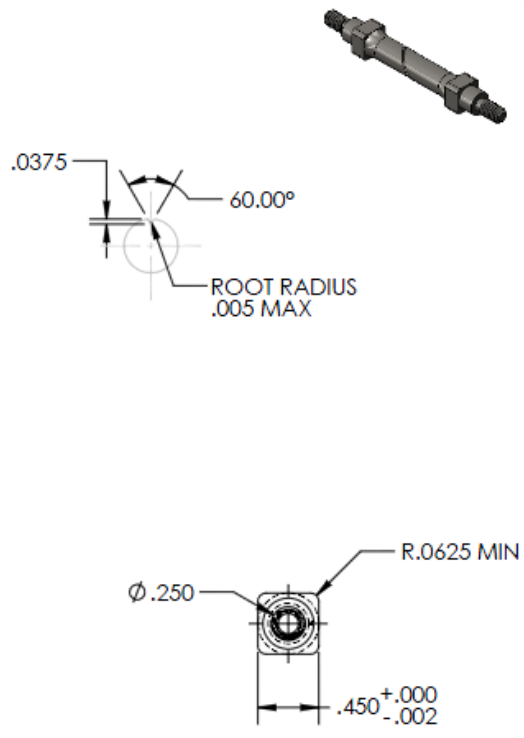

Fig. 13. Geometry details of the SS304/308 weld steel SNTT specimen. 


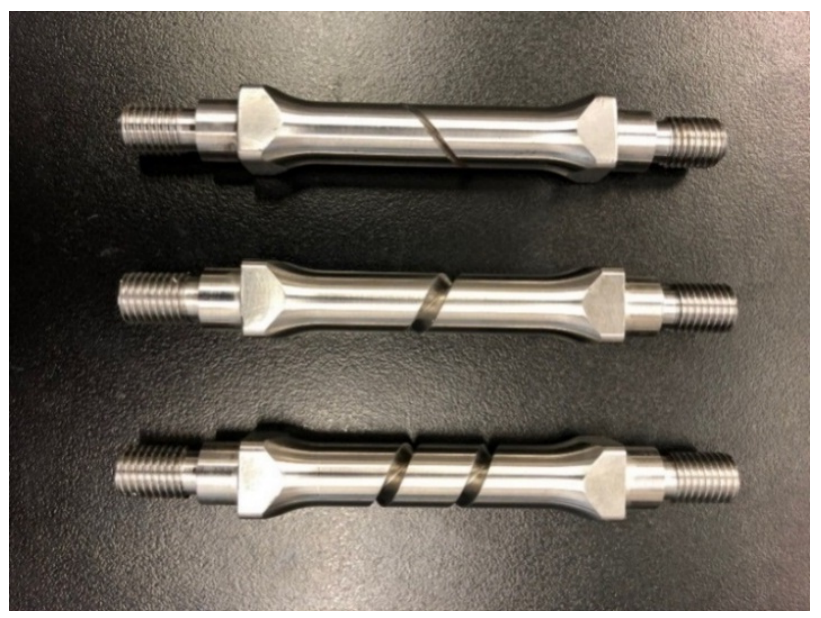

Fig. 14. SNTT specimens with different spiral groove designs, (Top) single loop spiral groove with 0.0375" notch depth, (Middle) single loop with 0.100" notch depth, (Bottom) two loops with 0.1125 " notch depth.

The weld specimen has its centerline aligned with the center of FZ; the associated gage section, and the actual dimension and location of the SNTT sample in the as-received Sandia weldment was marked with blue stripe, are shown in Fig. 15. The spiral groove is located within the gage section, with the targeted notch depths.

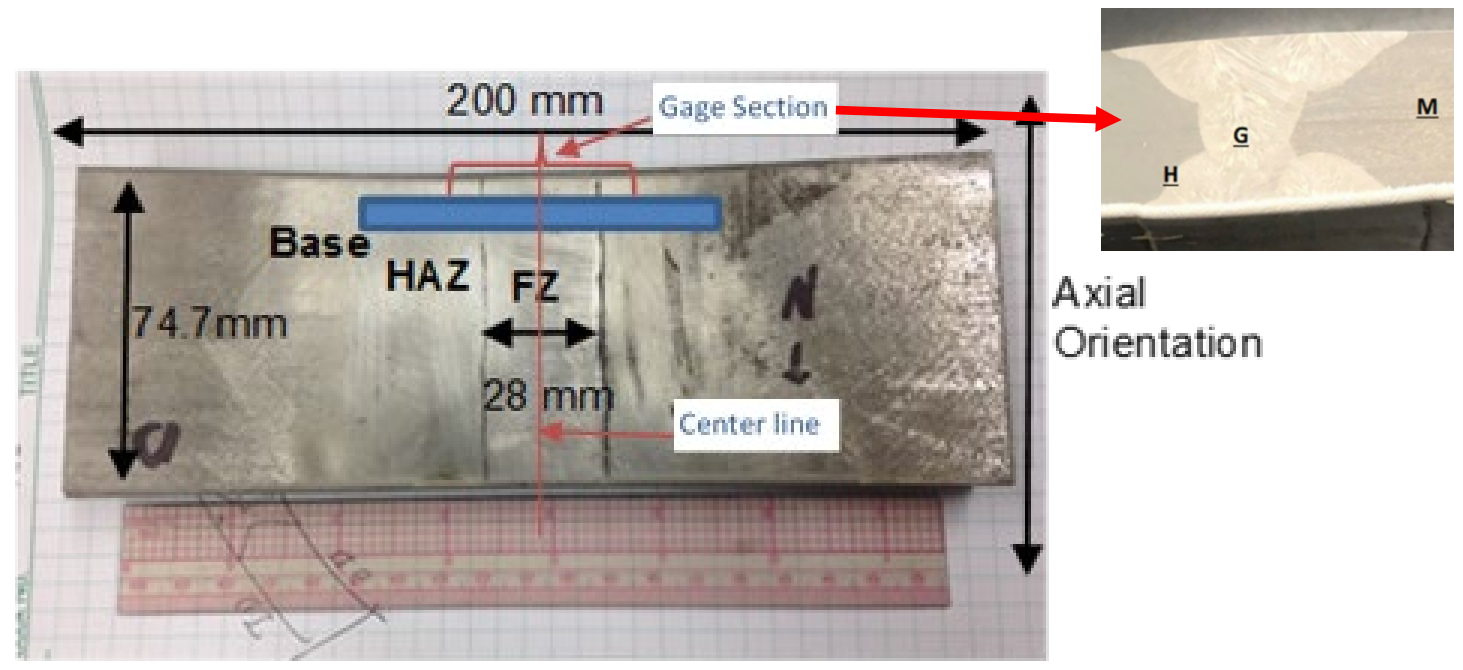

Fig. 15. SNTT weld sample machining physical location in the as-received Sandia weldment.

The HAZ specimen has its centerline aligned with the edge of FZ; the associated gage section, and the actual dimension and location of the SNTT sample in the as-received Sandia weldment was marked with the blue stripe, are shown in Fig. 16. 


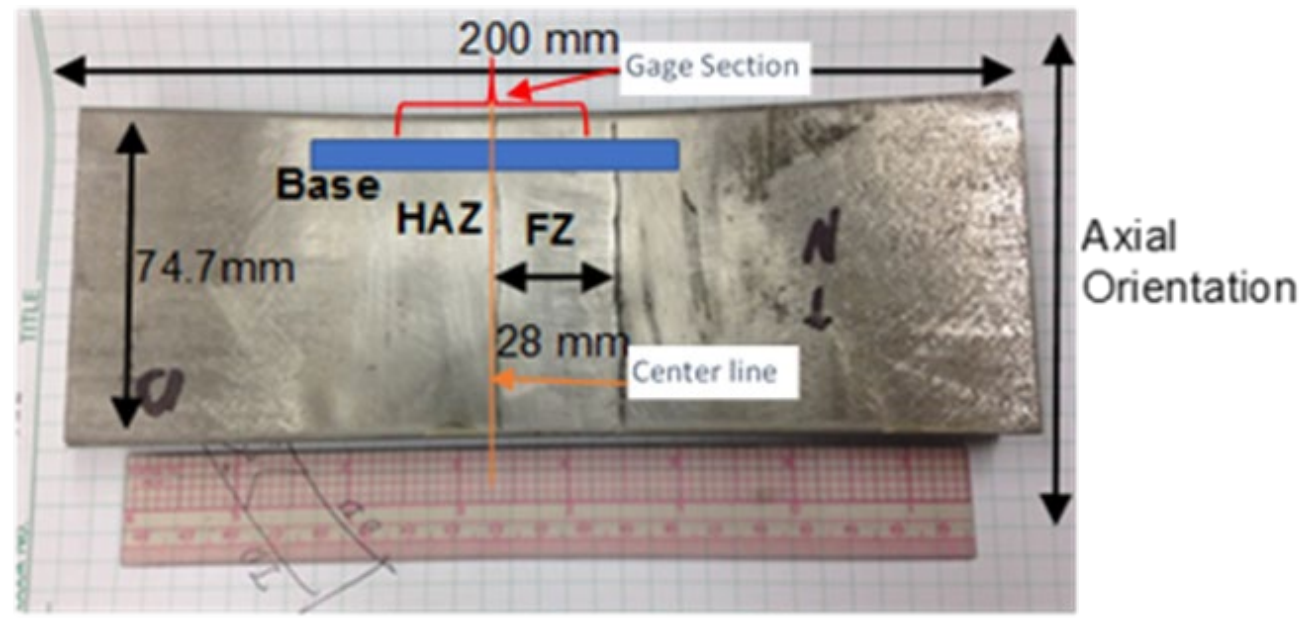

Fig. 16. SNTT HAZ sample machining physical location in the as-received Sandia weldment.

\subsection{SS304/308 Weld SNTT Sample Fixture Design Configuration}

Two major concerns were addressed in the fixture and base design of SS304/308 weld SNTT samples. Because a high number of cycles were involved in the fatigue pre-crack process, threads could be coupled with bolts to stabilize the specimens. Therefore, small fixtures were designed separated to accommodate the SS304/308 weld SNTT samples (Fig. 17), including a base was designed to connect the SNTT specimen fixture to the biaxial tester machine (Fig. 18). In both the fixture and base, rotated bolt arrays were applied to secure the fastening between different components.
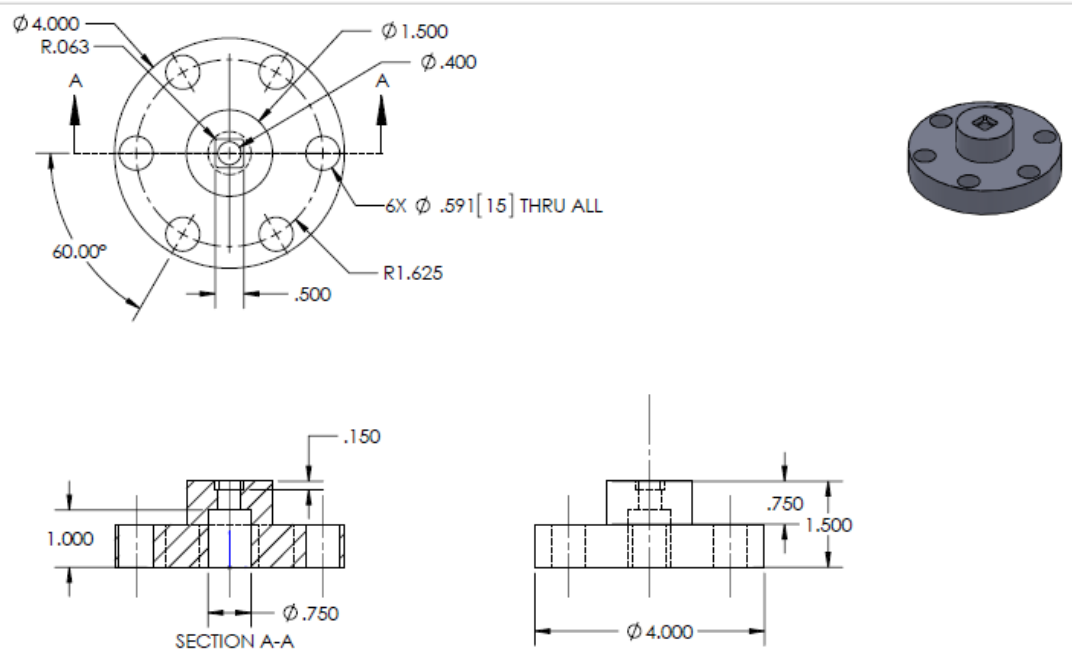

Fig. 17. Schematic for fixture to adapt the 304/308 weld SNTT specimen. 


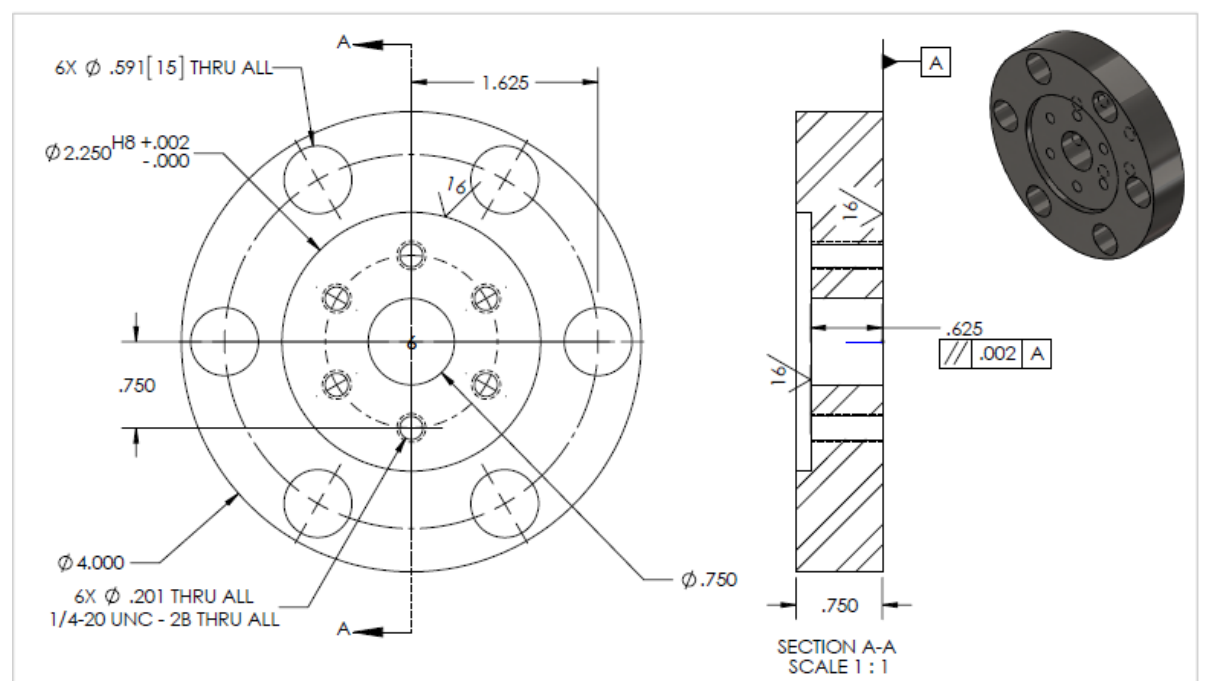

Fig. 18. Schematic for base to adapt the SS304/308 weld SNTT fixture.

\subsection{SNTT Equipment Setup}

The SNTT testing of SS304/308 weld steel is primarily focused on samples machined from the asreceived weld plates from Sandia mock-up canister weldment. Preliminary calculations estimate that the threshold of crack initiation in these samples with shallow notch of 0.0375 " is around $280 \mathrm{lbf}$-in. The maximum capacity of the torque load provided by the Test Resource 830 axial-torsion machine is 1,620 lbf-in. The cyclic fatigue frequency can reach $10 \mathrm{HZ}$ range under the targeted torque load range. These specifications ensure that cycle fatigue testing of SNTT SS304/308 weld steel specimens can be conducted, in addition to the final SNTT fatigued sample fracture testing.

\subsection{SNTT Testing on SS304/308 Weld Steel Material}

\subsubsection{Cycle fatigue process}

The cycle fatigue process on SS304/308 weld SNTT samples were performed through the angle control mode by a function generator built in the TestResource control system. In order to find the fatigue threshold of the SS304/308 SNTT samples, the initial maximum torque was adjusted to approximately $270 \mathrm{lbf}-\mathrm{in}$ with $5 \mathrm{HZ}$ cyclic fatigue process. This cyclic load was gradually increased to facilitate the crack growth in a reasonably time frame to reach the targeted total crack growth length ("a", notch depth plus the fatigue crack growth length); where the targeted a/D is normally in the ranges of 0.35 to 0.45 . 
The crack growth during the fatigue cycles was monitored by the specimen's compliance or stiffness changes and then using the developed compliance function to estimate the crack penetration depth.

\subsubsection{Monotonic loading fracture test}

Fatigued SNTT sample was then loaded monotonically using the biaxial tester with series of loading/unloading sequences until failure; where the loading rate of $2.2 \mathrm{lbf}$-in/second and unloading rate of $17.7 \mathrm{lbf}$-in/second were used. During the monotonic loading/unloading period, the axial force is maintained at nil zero condition to ensure a pure torsion loading condition. The typical experimental test results for SNTT 304/308 weld samples are shown in Fig. 19 and Fig. 20.
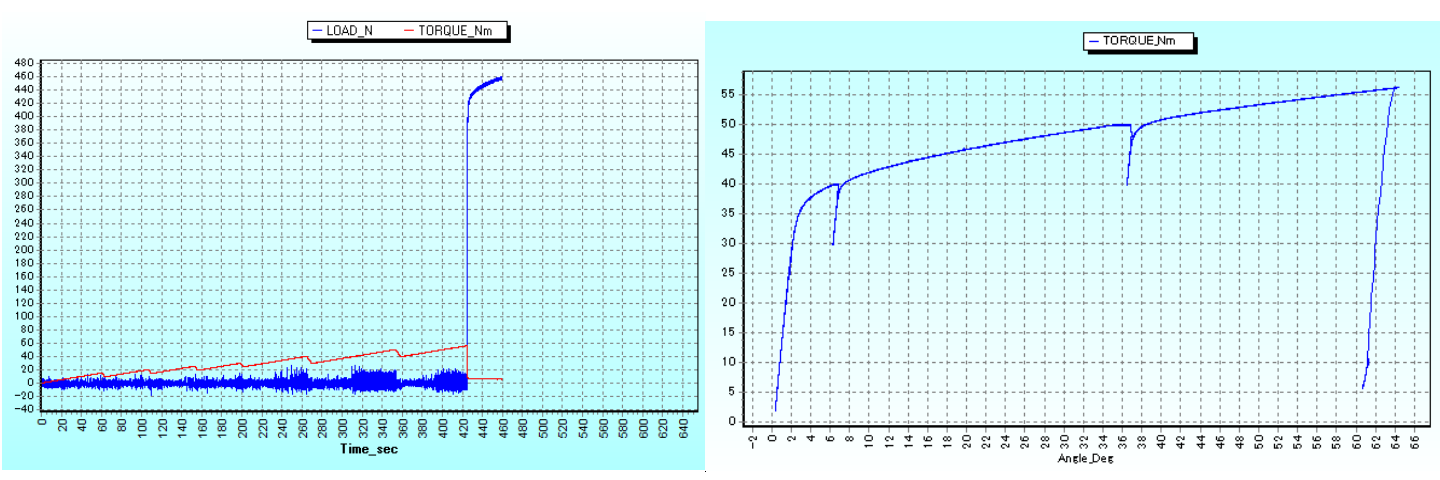

Fig. 19. HAZ-1 test results, (Left) upon sudden failure a significant shock reaction was observed, (Right) The slopes of different loading and unloading sequences does not change, indicate no crack growth during the monotonic loading, the specimen undergo significant non-linear deformation before final failure, at $56 \mathrm{~N}-\mathrm{m}$ (495 Lbf-in) fracture torque.
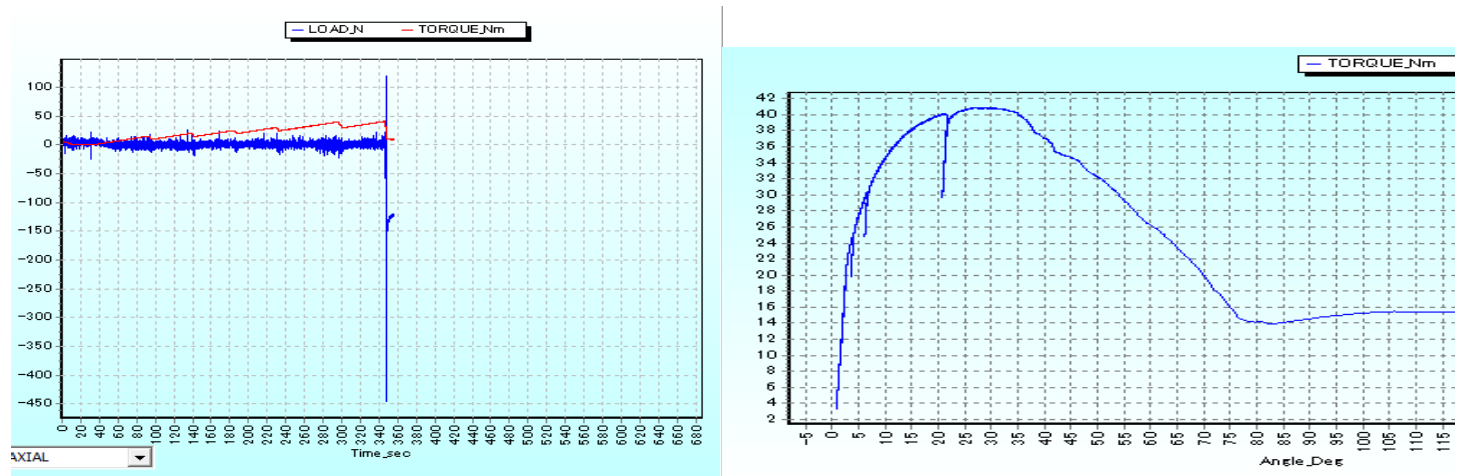

Fig. 20. 2-loop Weld-4 test results, (Left) upon sudden failure a significant shock reaction was observed, (Right) The slopes of different loading and unloading sequences does not change, indicate no crack growth during the monotonic loading, the specimen undergo significant non-linear deformation before final failure, at $41 \mathrm{~N}-\mathrm{m}$ (362.8 Lbf-in) fracture torque. 


\subsubsection{Failed sample characterization}

The failed SNTT samples were characterized using an optical camera, which captured optical images of the specimen and the fractured surfaces, as shown in Fig. 21 for the tested HAZ-1 SNTT sample and in Fig. 22 for the tested 2-loop Weld-4 SNTT sample. Fig. 21 shows that the fracture surface has smooth fatigue surface profile before final crack initiation under monotonic loading; where the HAZ-1 sample has total crack length ratio, a/D, of 0.35 .

Fig. 22 shows that the 2-loop Weld-10 SNTT sample fractured into two halves during the monotonic loading. The failed surfaces from matching surfaces indicate a significant crack tip blunting occurred before the specimen final fracture. The fatigue pre-crack length is at 0.0405 and the notch depth is at 0.1125 "; the total crack length ratio, a/D, is equal to 0.40 . The fractured surface profile shows smooth precrack growth surface and the final fast fracture topology characteristic under monotonic loading. The fracture initiation site is near the HAZ region away from the center of FZ.

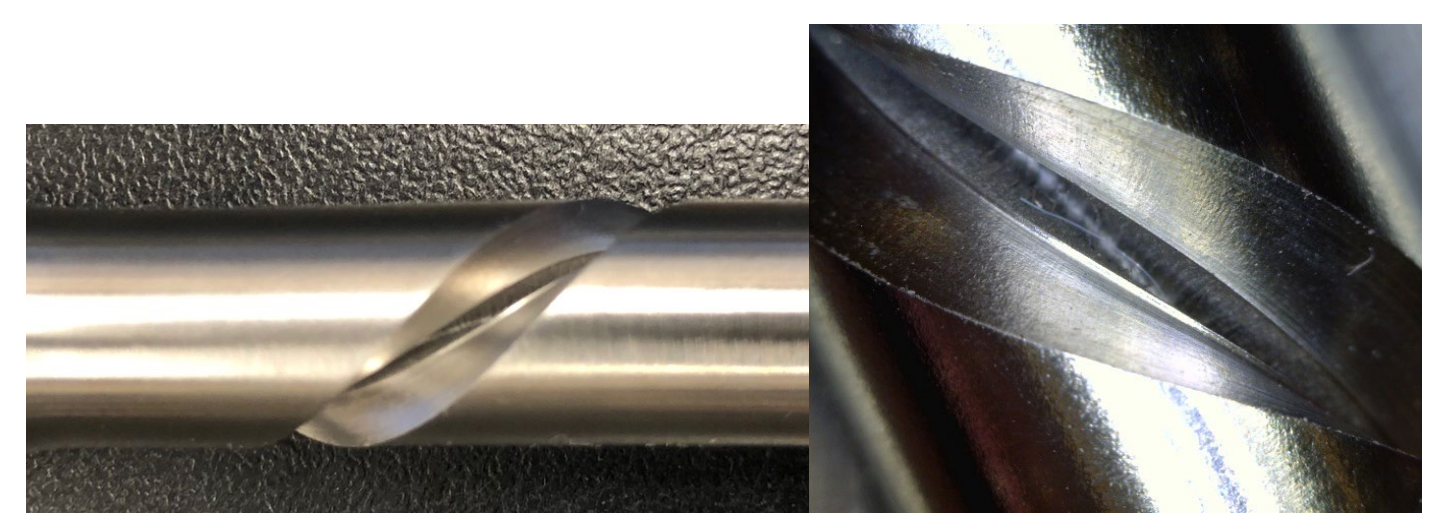

Fig. 21 SNTT HAZ-1 fracture specimen and the associated fracture surface profile 


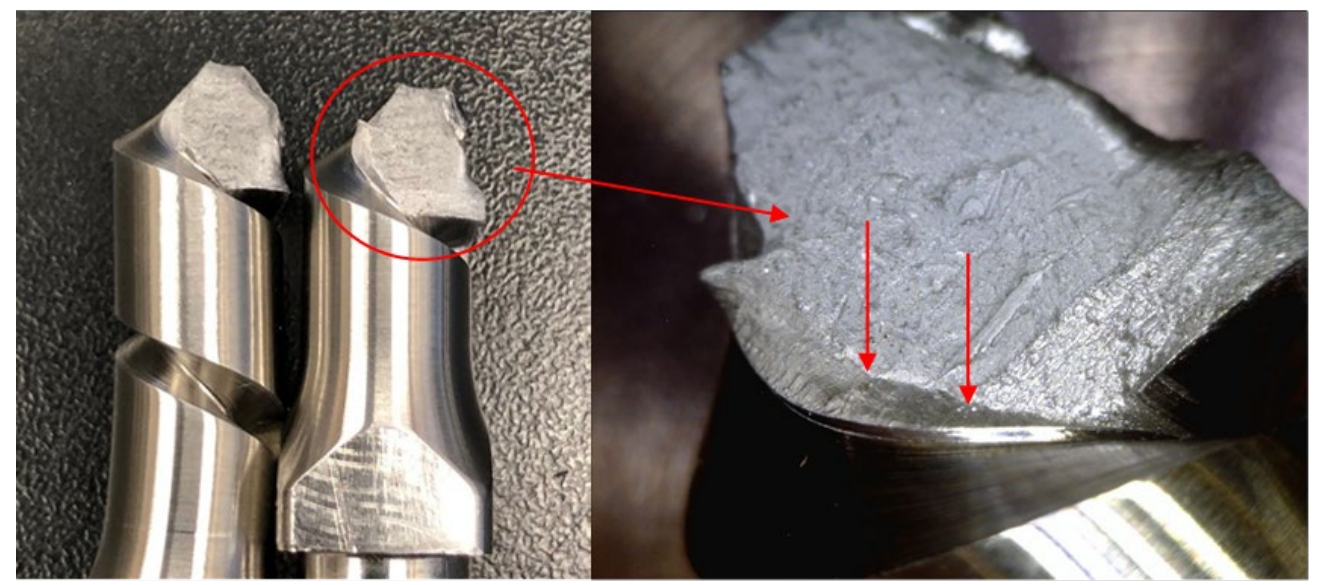

Fig. 22. SNTT 2-loop Weld-10 fracture specimen and the associated fracture surface profile, the red arrows point to the fatigue pre-crack front.

\subsubsection{SNTT 304/308 weld samples fracture test results}

The details of the SNTT 304/308 weld samples test results are illustrated in Table 1; where most weld specimens failures were initiated at HAZ region, and all the two loops weld samples were failed at or near HAZ regions, as shown in Fig. 23. Table 1 shows that the fracture torques from the different tests appear to be self-consistent at the targeted a/D ratio, which indicates the good repeatability of the SNTT methodology in applying to the highly ductile SS304/308 weld materials. The large axial bending distortion observed from the fractured 2-loop deep-notch specimens is due to significant axial load reaction shock upon SNTT specimen catastrophically fractured and broken into two pieces, as shown in Fig. 24.

Table 1 SNTT 304/308 weld specimens and 304 base specimen fracture test results

\begin{tabular}{ccccccccc}
\hline Sample & $\begin{array}{c}\text { Spiral } \\
\text { groove } \\
\text { loop }\end{array}$ & $\begin{array}{c}\text { Notch } \\
\text { depth, a } 0 \\
\mathrm{~mm}\end{array}$ & $\begin{array}{c}\text { Gage } \\
\text { length, } \\
\mathrm{GL} \\
\mathrm{mm}\end{array}$ & $\begin{array}{c}\text { Total } \\
\text { crack } \\
\text { length, } \mathrm{a}\end{array}$ & $\begin{array}{c}\text { a/D } \\
\mathrm{mm}\end{array}$ & $\begin{array}{c}\text { Uncracked } \\
\text { ligament, } \\
\mathrm{b}\end{array}$ & $\begin{array}{c}\text { Crack } \\
\text { front } \\
\text { length, B* }\end{array}$ & $\begin{array}{c}\text { Fracture } \\
\text { Torque }\end{array}$ \\
Weld-1 & 1 & 1.91 & 23.92 & 3.34 & 0.350 & 6.19 & 25.55 & 56.0 \\
Weld-5 & 1 & 2.54 & 13.46 & 4.23 & 0.440 & 5.30 & 13.87 & 38.5 \\
Weld-8 & 2 & 2.86 & 23.92 & 3.96 & 0.416 & 5.56 & 25.95 & 39.5 \\
Weld-9 & 2 & 2.86 & 23.92 & 4.00 & 0.420 & 5.52 & 25.77 & 38.2 \\
Weld-10 & 2 & 2.86 & 23.92 & 3.89 & 0.408 & 5.64 & 26.33 & 41.0 \\
Base & 2 & 2.86 & 23.92 & 3.77 & 0.396 & 5.75 & 26.97 & 45.0 \\
\hline
\end{tabular}

*Single loop B: $\sqrt{ }\left[(2 \pi(\mathrm{R}-\mathrm{a}))^{2}+\mathrm{GL}^{2}\right]$,

*Double loop B: $\sqrt{[}\left[(2 \pi(\mathrm{R}-\mathrm{a}))^{2}+\mathrm{GL}^{2}\right], \mathrm{R}$ is radius. 


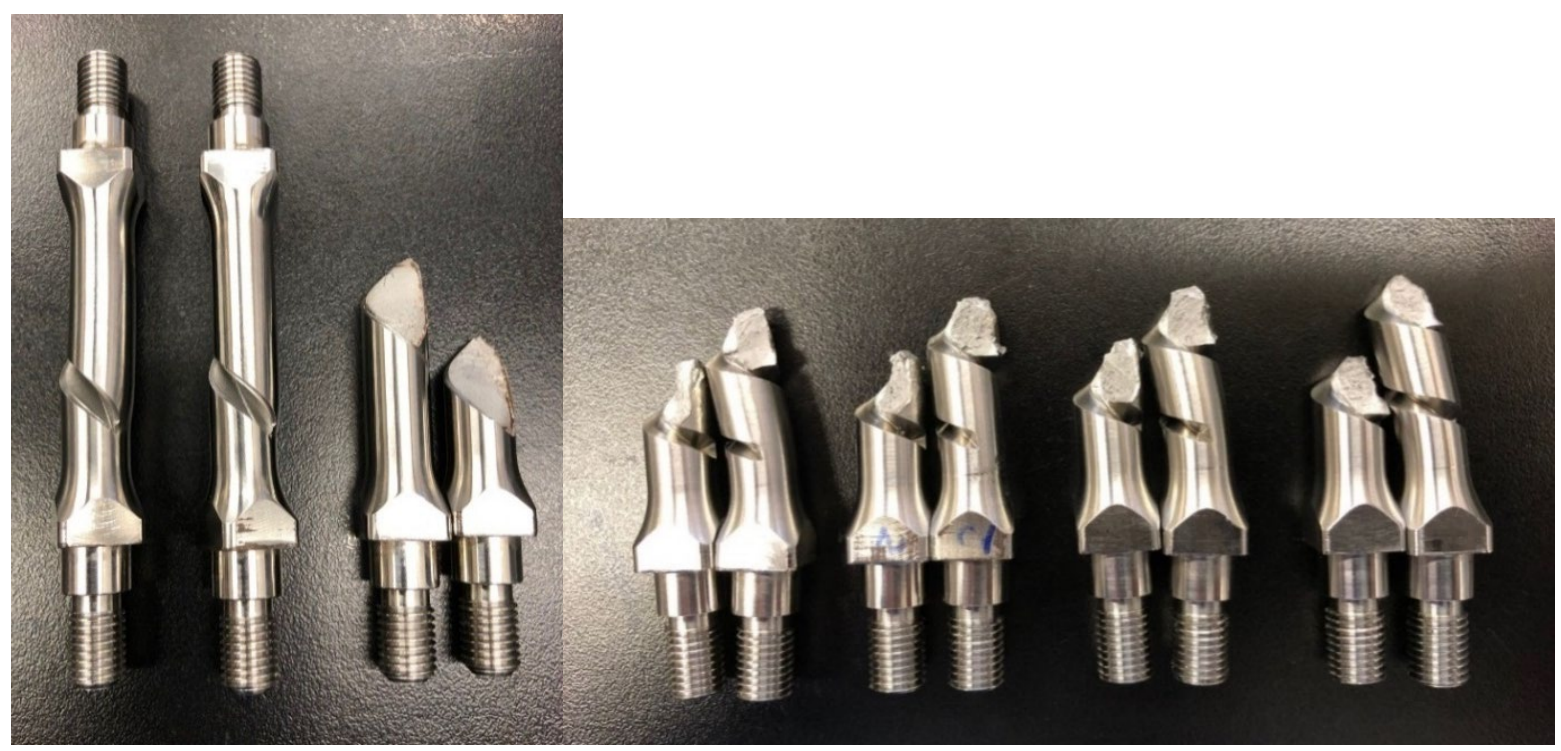

Fig. 23. (Left) Most SNTT weld 1-loop shallow-notch specimens' failures are initiated at HAZ regions, (Right) All the weld 2-loop deep-notch specimens are failed at HAZ regions.
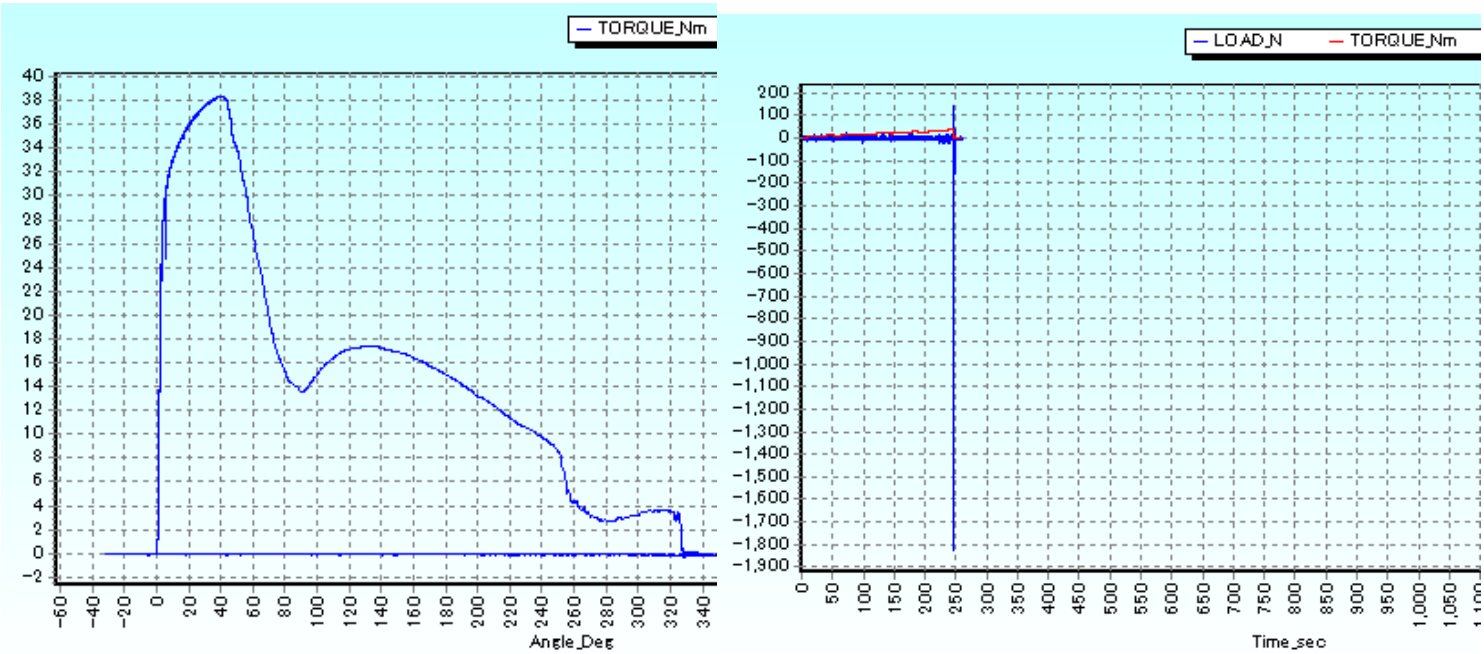

Fig. 24. SNTT Weld-9 specimen fracture test results, (Left) Torque vs. angle data, and (Right) Upon failure significant axial load shock was observed, reaching magnitude of 1800 N.

\subsection{Fracture toughness evaluation on the tested SNTT weld samples}

\subsubsection{FEM analyses and energy release rate evaluation for SNTT Weld-10 specimen test results}

The finite element model used for evaluating the apparent energy release rate or $J_{Q}$ is shown in Fig. 25, 
where 89,603 nodes and 21,280 3-D solid reduced-integration elements were used to model SNTT Weld10 specimen with a crack length of $0.153 \mathrm{inch}$. The fracture torque is at $362.8 \mathrm{lbf}-\mathrm{in}$. The deformed FEM model upon failure and the estimated von Miss stress contours are shown in Fig. 26. The Abaqus Jcontour integral routine with 9-contours option was used to determine J value. Near middle layer's Jcontour data were used to estimate $\mathrm{J}_{\mathrm{Q}}$ for SNTT 304/308 Weld-10 specimen upon final fracture; which results in $\mathrm{J}_{\mathrm{Q}}=850 \mathrm{lb} / \mathrm{in}\left(148.8 \mathrm{KJ} / \mathrm{m}^{2}\right)$.

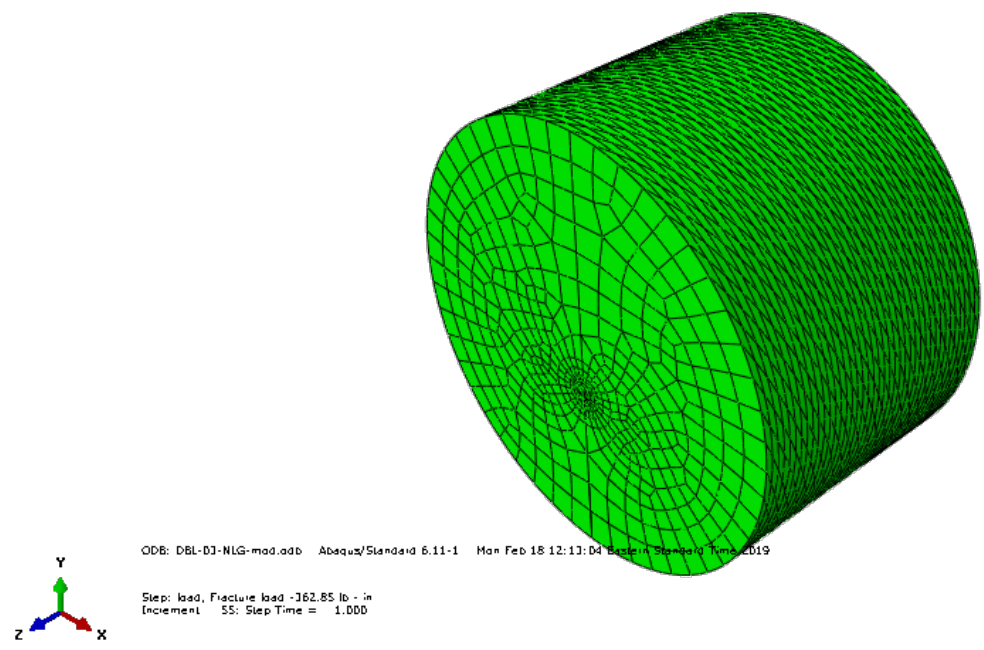

Fig. 25. Finite element model for SNTT Weld-10 specimen test simulation.
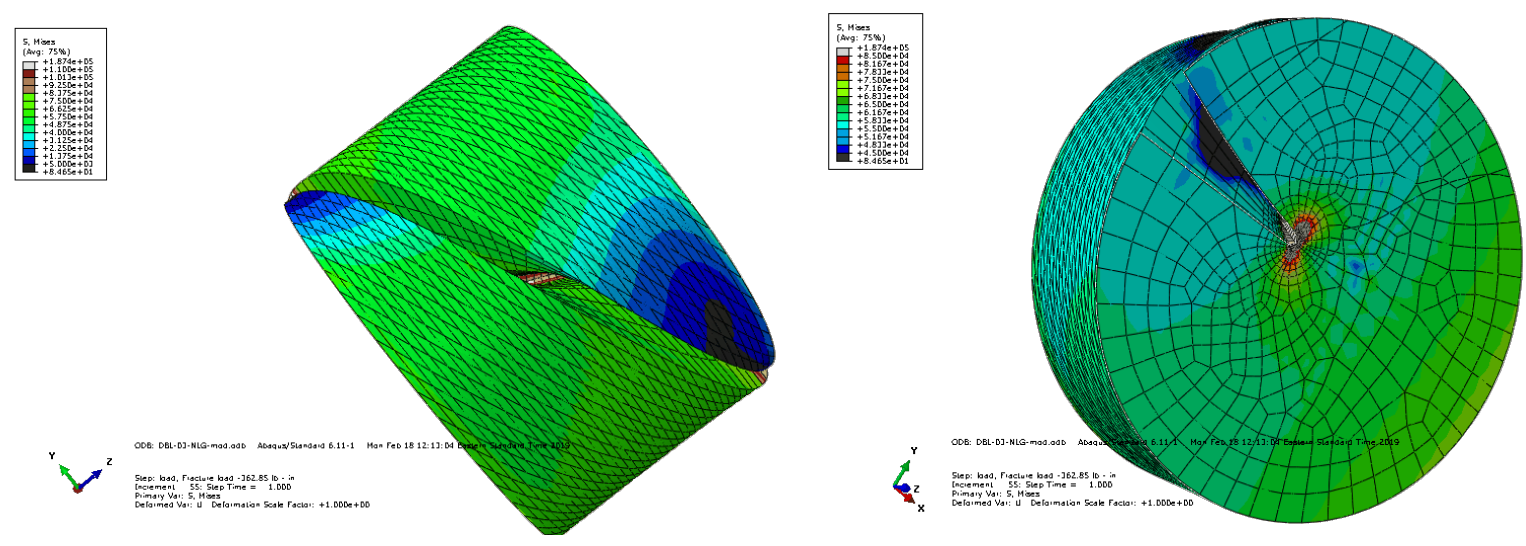

Fig. 26. (Left) Full model FEM deformation and von-Miss stress profile, (Right) Deformation and von Miss stress profiles at near middle layer of finite element model. 


\subsubsection{J-Integral evaluation for SS 304/308 weldment material}

The J-integral evaluation results based on $J=\frac{1}{B b} \int_{0}^{\theta_{c}} T_{q} d \theta_{c}$ for the SS304/308 weld SNTT samples and SS304 base SNTT sample are illustrated in Table 2. Where load-displacement area under torque load rotation angle trend curve is shown in Fig. 27, the associated J-integral value evaluation is shown in Table 2. Table 2 shows the mean J-integral upon fracture for SS304/308 weld and SS304 base are $144.2 \mathrm{KJ} / \mathrm{m}^{2}$ and $459.0 \mathrm{KJ} / \mathrm{m}^{2}$, respectively.

Table 2 SNTT 304/308 weld \& SS304 base specimens fracture toughness test results

\begin{tabular}{|c|c|c|c|c|c|c|c|c|c|c|}
\hline $\begin{array}{c}\text { Sample } \\
\text { ID }\end{array}$ & $\begin{array}{l}\text { Spiral } \\
\text { groove } \\
\text { loop }\end{array}$ & $\begin{array}{l}\text { Notch } \\
\text { depth, a } a_{0} \\
\text { mm }\end{array}$ & $\begin{array}{c}\text { Gage } \\
\text { length, GL } \\
\mathrm{mm}\end{array}$ & $\begin{array}{c}\text { Total crack } \\
\text { length, a } \\
\text { mm }\end{array}$ & $\mathrm{a} / \mathrm{D}$ & $\begin{array}{c}\text { Uncracked } \\
\text { ligament, b } \\
\mathrm{mm}\end{array}$ & $\begin{array}{c}\text { Crack front } \\
\text { length, B } \\
\text { mm }\end{array}$ & $\begin{array}{c}\text { Fracture } \\
\text { Load, Tq } \\
\text { N-m }\end{array}$ & $\begin{array}{c}\mathrm{Tq}-\theta \\
\text { area, A } \\
\text { KJ }\end{array}$ & $\begin{array}{c}\text { J-integral, } \\
\mathrm{A} / \mathrm{bB} \\
\mathrm{KJ} / \mathrm{m}^{2}\end{array}$ \\
\hline Weld-1 & 1 & 1.91 & 23.92 & 3.34 & 0.350 & 6.19 & 25.55 & 56.0 & 24.50 & 154.9 \\
\hline Weld-5 & 1 & 2.54 & 13.46 & 4.23 & 0.440 & 5.30 & 13.87 & 38.5 & 9.56 & 130.1 \\
\hline Weld-8 & 2 & 2.86 & 23.92 & 3.96 & 0.416 & 5.56 & 25.95 & 39.5 & 20.52 & 142.3 \\
\hline Weld-9 & 2 & 2.86 & 23.92 & 4.00 & 0.420 & 5.52 & 25.77 & 38.2 & 22.92 & 154.0 \\
\hline \multirow[t]{3}{*}{ Weld-10 } & 2 & 2.86 & 23.92 & 3.89 & 0.408 & 5.64 & 26.33 & 41.0 & 20.77 & 139.9 \\
\hline & & & & & & & & & Mean & 144.2 \\
\hline & & & & & & & & & Two Sigma & \pm 10.4 \\
\hline $\begin{array}{c}\text { SS304 } \\
\text { Baseline }\end{array}$ & 2 & 2.86 & 23.92 & 3.77 & 0.396 & 5.75 & 26.97 & 45.0 & 71.25 & 459.0 \\
\hline
\end{tabular}

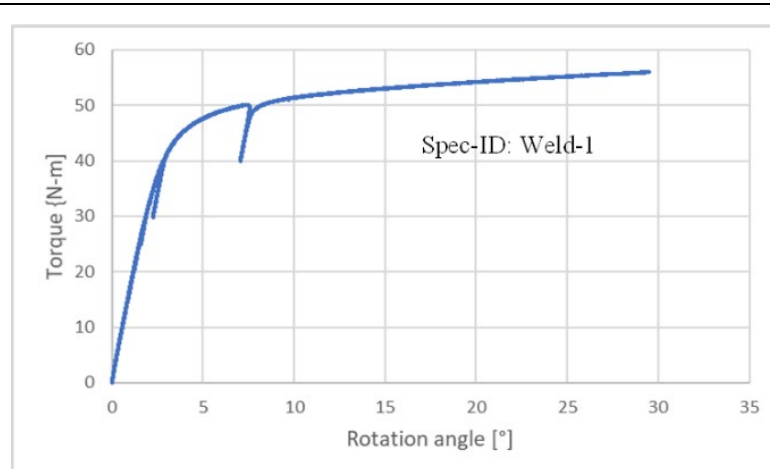

Maximum torque $=56.0 \mathrm{Nm}$

Area $\left(\mathrm{N}-\mathrm{m}\right.$ deg. $\left.{ }^{\circ}\right)=1403.7 \mathrm{KJ}-\mathrm{deg} .^{\circ}$

Area $(\mathrm{N}-\mathrm{m}$ rad. $)=24.5 \mathrm{KJ}$-radian

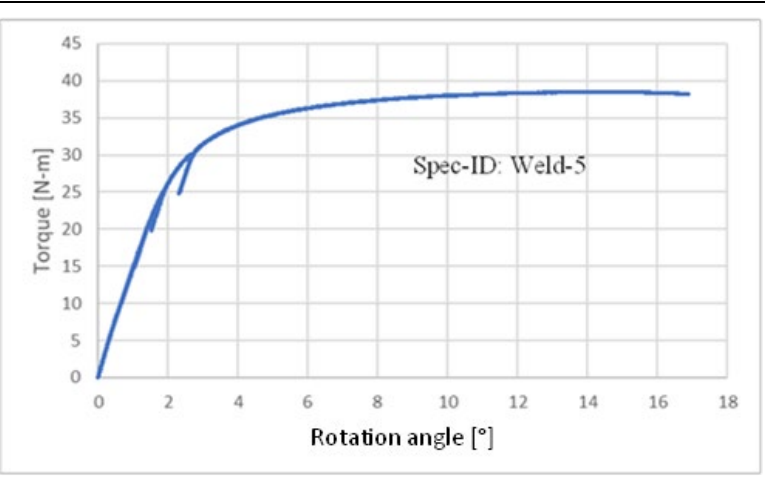

Maximum torque $=38.5 \mathrm{Nm}$

Area $\left(\mathrm{N}-\mathrm{m}\right.$ deg. $\left.{ }^{\circ}\right)=547.7 \mathrm{KJ}-\mathrm{deg} .^{\circ}$

Area $(\mathrm{N}-\mathrm{m}$ rad. $)=9.56 \mathrm{KJ}$-radian 


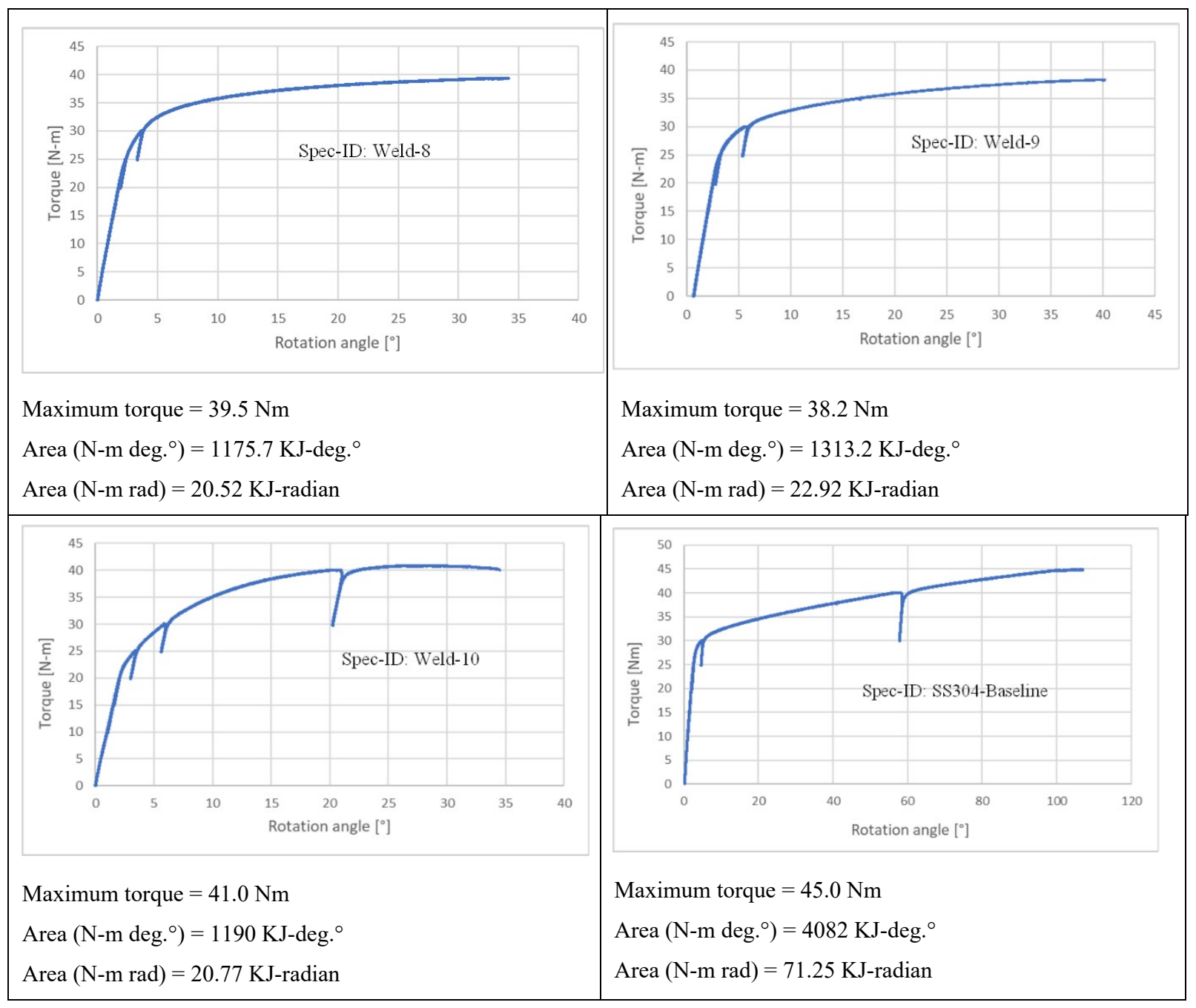

Fig. 27. SNTT test torque load versus rotation angle trend curve up to maximum fracture torque

\subsubsection{J-integral evaluation comparison between SNTT and Compact Tension (CT) tests results}

The comparison of SNTT 304/308 weld fracture toughness (represented with J-integral value at final fracture load, $\mathrm{J}_{\mathrm{Q}}$ ) and the mean $\mathrm{J}_{\mathrm{IC}}$ obtained from conventional CT test [25] are illustrated in Table 3. Table 3 shows that the SNTT estimated $\mathrm{J}_{\mathrm{Q}}$, is very close to the mean $\mathrm{J}_{\mathrm{IC}}$ obtained from the CT method for the weld specimens. Base metal CT test fracture toughness appears to be higher than that estimated from SNTT approach; this could be due to the inherited canister wall cold work forming process of the received canister weldment. The high uncertainty for both base and weld metals from CT tests were also observed. Due to limit SNTT base metal test conducted, no associated uncertainty study was carried out. 
Small two-sigma uncertainty bond of SNTT approach for weld samples compared to that of CT test results could be primary due to SNTT inherited high geometry constraint and the self-consistent fracture torques observed from the SNTT fracture test results as shown in Tables 1.

Table 3 Summary of fracture toughness obtained from SNTT test and CT test results

\begin{tabular}{lllllll}
\hline Method & Material & Condition & $\begin{array}{l}\text { Temperature } \\
\mathrm{C}^{\circ}\end{array}$ & $\begin{array}{l}\text { Mean } \mathrm{J}_{\mathrm{IC}} \\
\mathrm{KJ} / \mathrm{m}^{2}\end{array}$ & $\begin{array}{l}\mathrm{J}_{\mathrm{Q}^{\prime}} \\
\mathrm{KJ} / \mathrm{m}^{2}\end{array}$ & $\begin{array}{l}\text { 95\% Bond } \\
\mathrm{KJ} / \mathrm{m}^{2}\end{array}$ \\
\hline CT & 304 base & Base metal & 21 & 672.0 & & 215.0 \\
SNTT & 304 base & Base metal & 21 & & 459.0 & \\
CT & $304 / 308$ weld & SAW & 21 & 147.0 & & 67.0 \\
SNTT & $304 / 308$ weld & SAW & 21 & & 144.2 & 10.4 \\
\hline
\end{tabular}




\section{FRACTURE TOUGHNESS EVALUATION FOR THIN-SHELL CLAD-PELLET INSERTS STRUCTURE}

\subsection{Mode I + Mode III mixed-mode fracture toughness evaluation}

SNTT method has been performed on mixed-mode fracture toughness evaluations [12-14,17] under pure torsion loading. For a 7475-T7351 aluminum, toughness evaluation [12], at $45^{\circ}$ spiral crack front, yields $\mathrm{J}_{\mathrm{II}} / \mathrm{J}_{\mathrm{I}}=0.72 \%$ and $\mathrm{J}_{\mathrm{III}} / \mathrm{J}_{\mathrm{I}}=0.06 \%$, where $99.2 \%$ of J-integral value is from $\mathrm{J}_{\mathrm{I}}$; and in other case of spiral crack front pitch angle deviated from $45^{\circ}$, yields $\mathrm{J}_{\mathrm{II}} / \mathrm{J}_{\mathrm{I}}=0.02 \%$ and $\mathrm{J}_{\mathrm{III}} / \mathrm{J}_{\mathrm{I}}=8.98 \%$, indicates a substantial increase in Mode III contribution, while as Mode II's contribution dropping to nil zero. In addition to the pure torsion loading study, an energy method for predicting fatigue life, crack orientation, and crack growth under multiaxial (tension/compression and torsion) loading conditions was also developed [26].

In a mixed-mode fracture toughness study [27], which utilized a complex test set-up with a specially machined CT specimen, test results indicate that Mode I + Mode III fracture toughness and tearing modulus reduced to 50 percent and 30 percent, respectively, compared to those under Mode I only for some ductile materials. Recently, an innovative edge notch disc bend (ENDB) method was used for Mode I + Mode III fracture toughness study for brittle materials [28-30]. The pure mixed-mode (Mode I + Mode III) condition of ENDB method can only be existed at the disk center point location, due to inherited compression loading induced in-plane shear Mode II deformation throughout the tested disk besides the disk center, especially approaching ENDB disk boundary. Furthermore, from the ENDB numerical evaluation results indicate that Mode II stress intensity factor existed throughout the ENDB sample and increased significantly further away from the disk center [31]. This phenomenon could be interpreted as a specimen size/geometry effect, thus, relatively large ENDB disk sample or other numerical correction scheme maybe required to mitigate this disk boundary effect for applying ENDB approach to Mode I + Mode III failure analyses. This is in contrast to the SNTT approach where Mode I + Mode III deformation with constant torque loading condition existed throughout the test sample gage section along the crack front. From the early study [12] indicates that the Mode II contribution to the mixed-mode fracture toughness of SNTT sample under pure torsion loading is considered negligible.

For thin shell clad SNTT sample with through thickness crack front under torsion loading, two schematic diagrams (Fig. 28) of hollow tubes under pure torsion loading were used to illustrate the deformation behaviors of spiral notched SNTT clad sample. Fig. 28 shows that under pure torsion loading, only the 

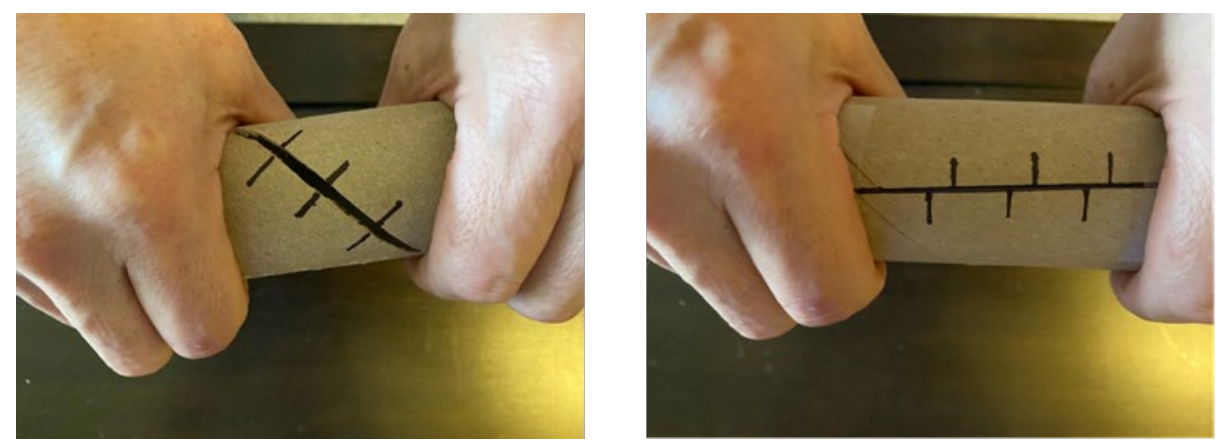

Fig. 28. (Left) Opening tensile mode I observed from $45^{\circ}$ pitch angle spiral cut slit; (Right) Out-of plane shear mode III observed from $0^{\circ}$ pitch angle cut slit (Along axial orientation) under pure torsion.

tensile opening Mode I displacement can be observed from a $45^{\circ}$ pitch angle spiral cut slit and only out of plane shear Mode III displacement can be observed from a $0^{\circ}$ pitch angle cut slit. For a thin shell structure, in plane shear Mode II failure requires much higher energy compared to that of opening tensile Mode I and out of plane shear Mode III, especially for a pure torsion loading of SNTT sample Mode II contribution to the fracture toughness is considered secondary. Thus, the mixed mode (Mode I + Mode III) failure behavior can be studied using SNTT method by varying the pitch angle of the spiral crack front deviated from the $45^{\circ}$ pitch angle.

\subsection{Zr-4 clad SNTT specimen preparation and biaxial tester set-up}

\subsubsection{Received AVERA Zr-4 clad tensile property}

Room temperature axial tensile tests were performed on unirradiated stress-relief annealed Zr-4 cladding samples received from AVERA using ASTM E 8M-04 standard, with the target strain rates at $0.001 \mathrm{~s}-1$ and $0.0003 \mathrm{~s}-1$. This $\mathrm{Zr}-4$ cladding material was used in the SNTT fracture toughness evaluation test. The stress-strain curve for the room temperature unirradiated cladding specimens is illustrated in Fig. 29. The $0.2 \%$ offset yield strength obtained from the tensile test of 152.4-mm long baseline Zr-4 cladding was 606 $\mathrm{MPa}$, the ultimate tensile strength was at $768 \mathrm{MPa}$, and the Young's modulus is $91 \mathrm{GPa}$.

\subsubsection{SNTT specimens designs and configurations}

SNTT samples were fabricated from a Zr-4 clad tubing, with diameter of 9.525-mm and thickness of $0.572-\mathrm{mm}$, and alumina pellet inserts with diameter of 8.0-mm and length of 15.24-mm (Fig. 30). The alumina-pellet inserts were bonded to $\mathrm{Zr}-4$ clad inner wall by using epoxy. There are two types of initial flaw/crack starter designs used in the fatigue pre-crack procedure, namely, a short and shallow $45^{\circ}$ notch machined from a 76.2-mm diameter diamond saw, and a through thickness hole at $0.762-\mathrm{mm}$ diameter, as 
illustrated in Fig. 31.
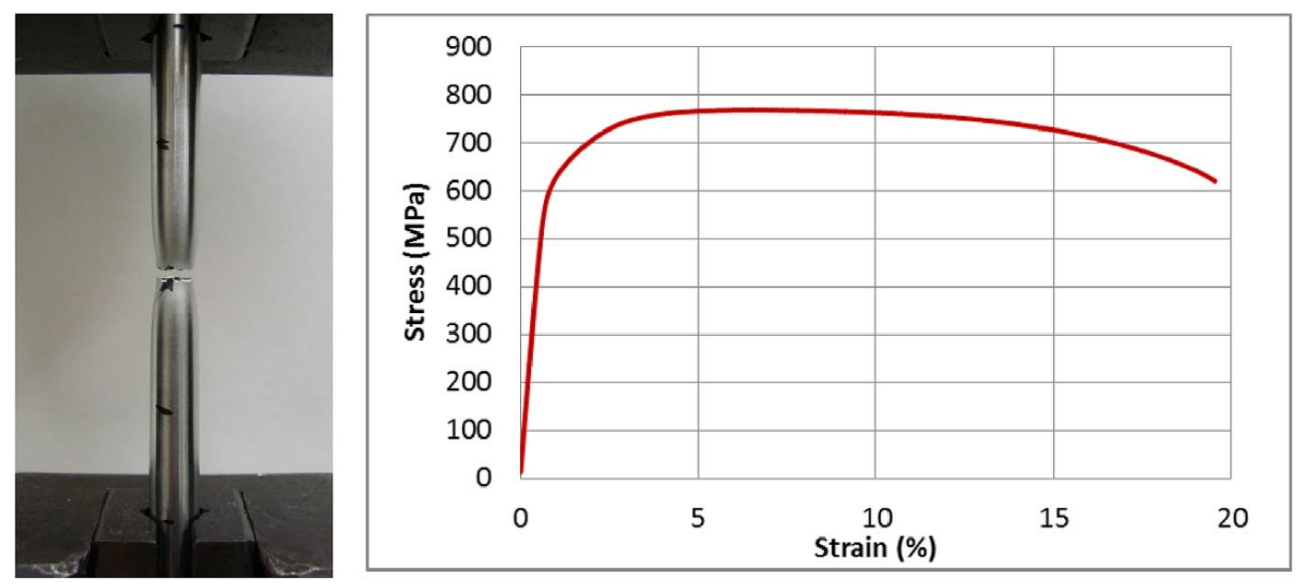

Fig. 29. A uniaxial tensile tube test and tensile stress-strain curve for received Zr-4 cladding.

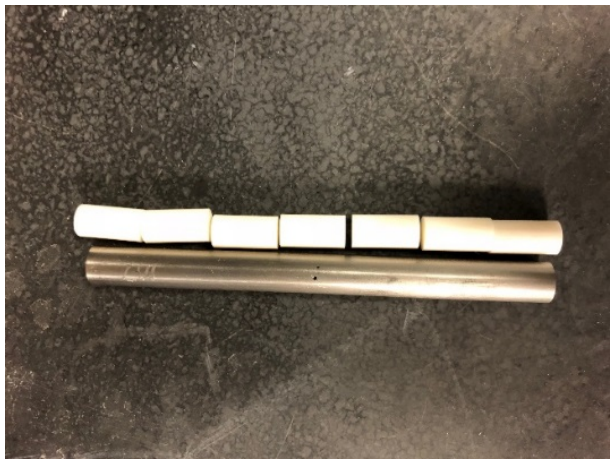

Fig. 30. Geometry details of SNTT specimen made of Zr-4 clad and alumina pellets.

\subsubsection{Zr-4 clad SNTT sample grip fixture design}

The SNTT clad sample end-grip design was based on the following consideration; (1) to effectively transfer the loading torque from the biaxial tester loading train to the SNTT specimen, and (2) to provide good protection to the SNTT specimen at grip ends. Because a high number of cycles were involved in the fatigue pre-crack process, a sufficient compressive contact pressure between grips and specimen is needed; this was achieved by a bolt tightening mechanism provided at the grips, in addition to the epoxy bonds. The detailed configuration of the grip design is shown in Fig. 32. 


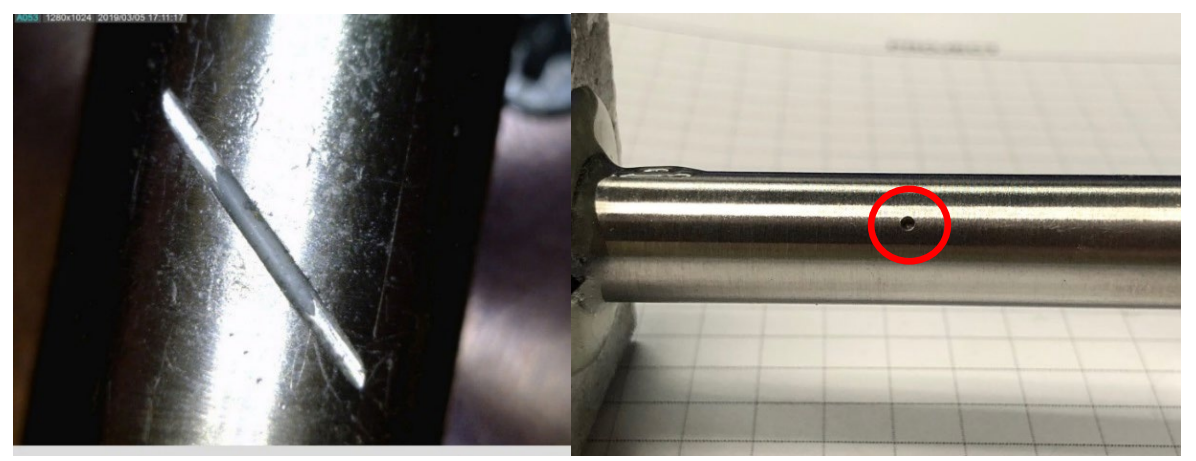

Fig. 31. (Left) SNTT specimen with a short $45^{\circ}$ notch, (Right) SNTT specimen with a 0.762-mm through clad thickness circular hole as crack starter.
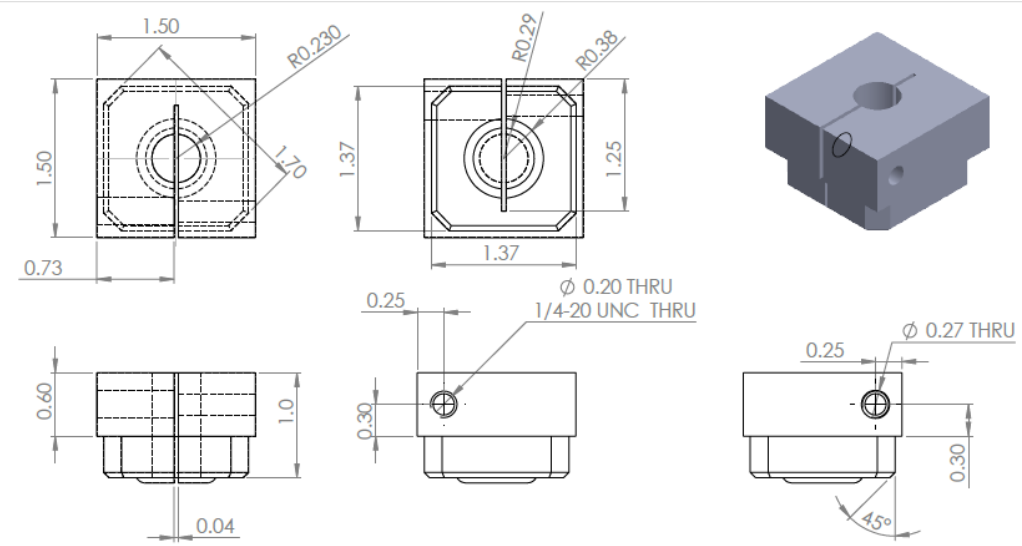

Fig. 32. Zr-4 clad SNTT sample grip design.

\subsubsection{SNTT equipment setup for Zr-4 clad sample testing}

Preliminary calculations estimate that the threshold of crack initiation in these samples with a short crack starter is around $11.3 \mathrm{~N}-\mathrm{m}$. The maximum capacity of the torque load provided by the Test Resource 830 axial-torsion machine is $183 \mathrm{~N}-\mathrm{m}$, which is sufficient to perform the final fracture test. The cyclic fatigue frequency can reach $10 \mathrm{~Hz}$ range under the targeted torque load range. These specifications ensure that cycle fatigue testing of $\mathrm{Zr}-4$ clad specimens can be conducted, in addition to the final fatigued sample fracture testing. The detailed SNTT biaxial tester set-up is shown in Fig. 33. 


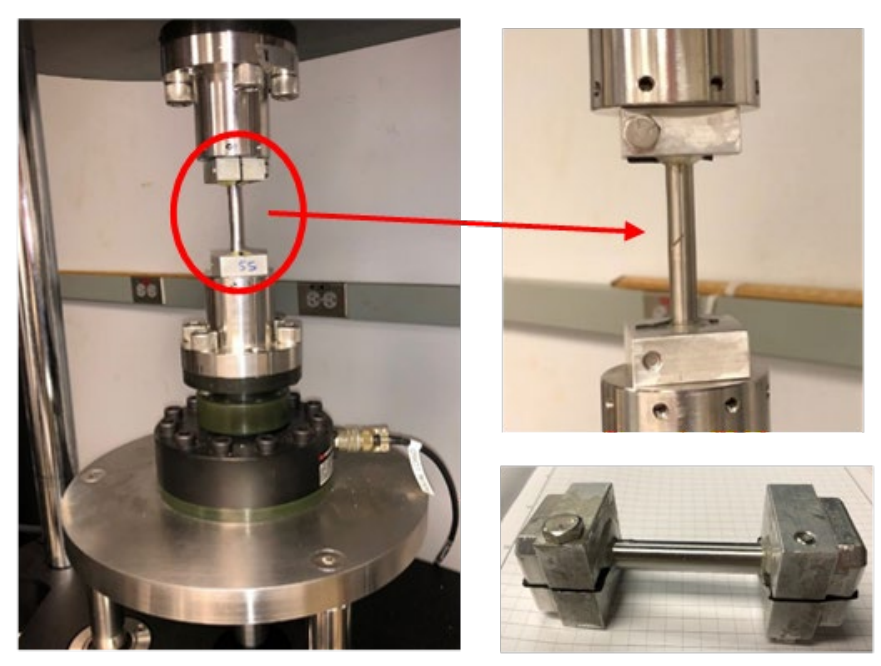

Fig. 33. (Left) SNTT biaxial tester set-up, (Right) SNTT clad specimen installation into biaxial tester torque ends, and SNTT sample made of Zr-4 clad/pellets rod and end-grips system.

\subsubsection{Pilot fatigue pre-crack testing on Zr-4 clad and SS clad SNTT specimens}

\subsubsection{Baseline Zr-4 clad SNTT specimen with notch flaw at $45^{\circ}$ pitch angle}

The preliminary estimate for the threshold of crack initiation was based on Zr-4 clad yield criterion on a SNTT sample surface, where the maximum shear stress profile for the notch cylinder specimen was estimated according to the simplified shear stress equations. Maximum allowable shear stress upon yield was estimated from the tensile yield stress of $\mathrm{Zr}-4$ clad. The torque load that can produce $60-80 \%$ of the maximum allowable shear stress on SNTT sample surface was used as the initial driving force to induce fatigue crack growth. The SNTT sample with an initial flaw/crack starter at length of 3.81-mm was used in the fatigue cyclic testing; where $11.3 \mathrm{~N}$-m cyclic torque loading was applied to the SNTT specimen. The final fatigue crack profile of the fatigued SNTT sample at the target crack length is shown in Fig. 34; where a sharp crack front was formed and its propagation orientation is perpendicular to the principal tensile stress profile.

\subsubsection{304 SS clad SNTT sample with a 0.762-mm through clad thickness circular hole}

The SNTT specimen with 0.762-mm circular hole as crack starter is shown in Fig. 35a. The specimen was undergone medium torque $(\sim 14.69 \mathrm{~N}-\mathrm{m})$ as the initial cyclic loading. At the intermediate fatigue process, the circular hole was elongated and evolved into a 1.524-mm elliptical hole with major axis perpendicular to the maximum tensile principal stress profile, as shown in Fig. 35b. Finally, the sharp crack front was 
emitting at the end of elliptical hole and perpendicular to the principal tensile stress profile as shown in Fig. 35c.
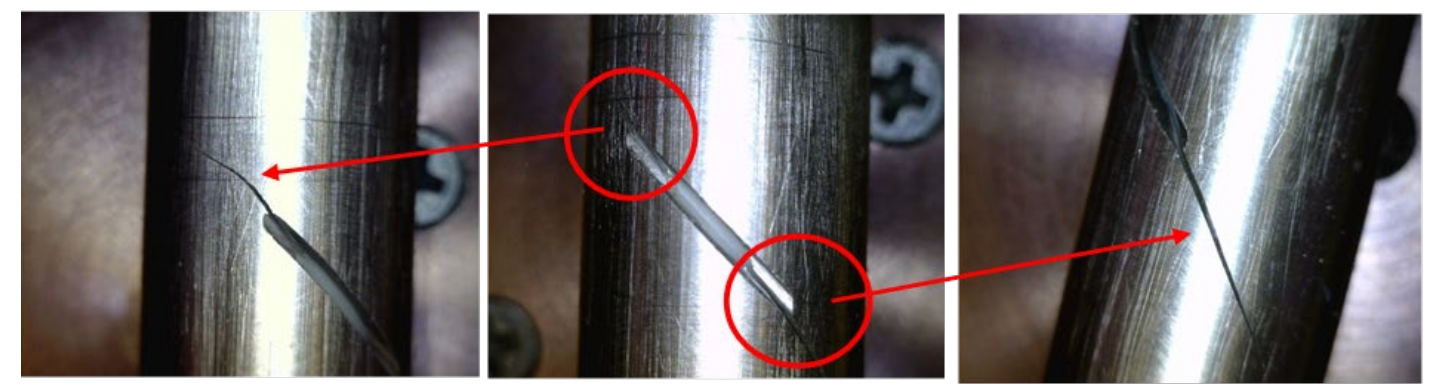

Fig. 34. The fatigue crack growth profile of Zr-4 clad SNTT sample under $11.3 \mathrm{~N}-\mathrm{m}$ torque cyclic loading.

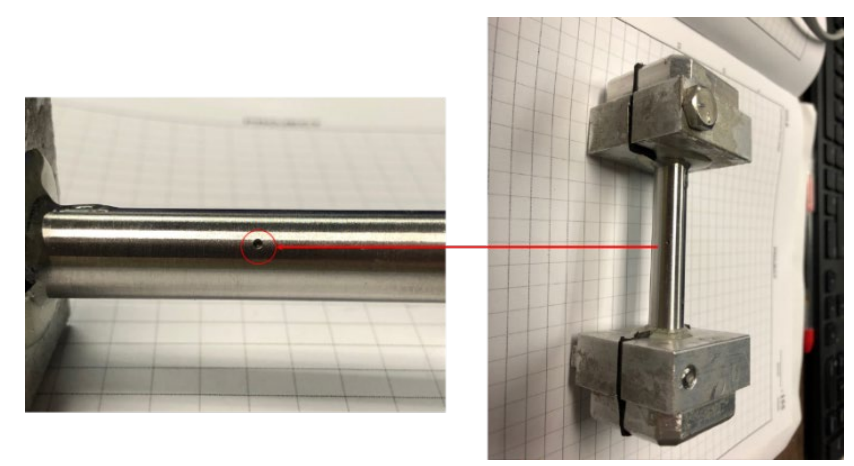

(a)

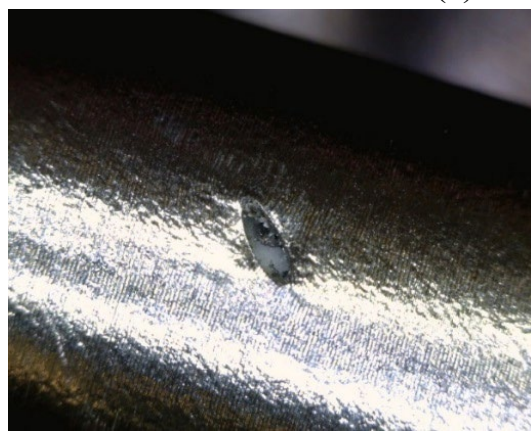

(b)

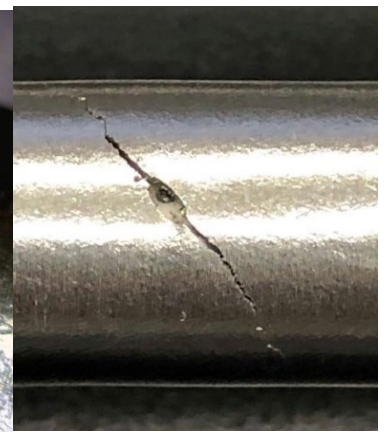

(c)

Fig. 35. (a) SS304 clad SNTT sample with 0.762- $\mathrm{mm}$ circular hole as crack starter; (b) under cyclic fatigue the circular hole elongated into 1.524-mm elliptical hole; (c) final fatigue crack growth on SNTT sample. 
5.2.5.3 Pre-hydride Zr-4 cad specimen with 0.762-mm circular hole and 0.381-mm shallow notch wings In order to understand SNF fatigue behavior, the pre-hydride Zr-4 clad SNTT sample with hydrogen content of $\sim 350$ wppm was used in the pin-hole fatigue evolution study. The details of $0.762-\mathrm{mm}$ diameter pin hole, with a pair of 0.381-mm notch wings, that is located on the opposite sides of the circular hole and is perpendicular to the principal tensile stress orientation, is shown in Fig. 36a. During the fatigue cycling no hole dimension increases was observed. The pre-hydride Zr-4 SNTT sample was fractured during the fatigue cycling process; and the postmortem examination indicated a brittle fracture failure mechanism without obvious fatigue pre-crack growth profile, as shown in Fig. 36b.

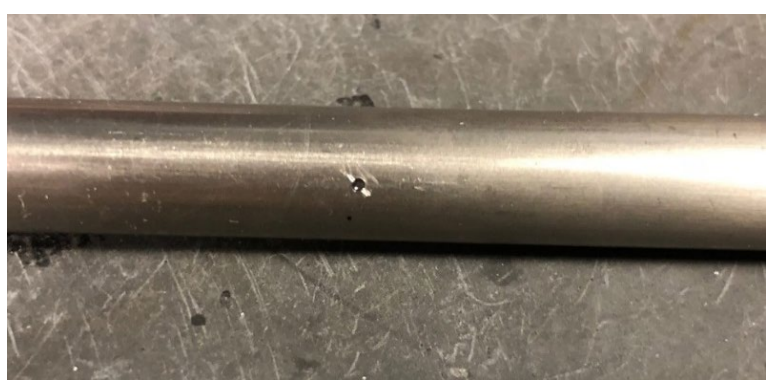

(a)

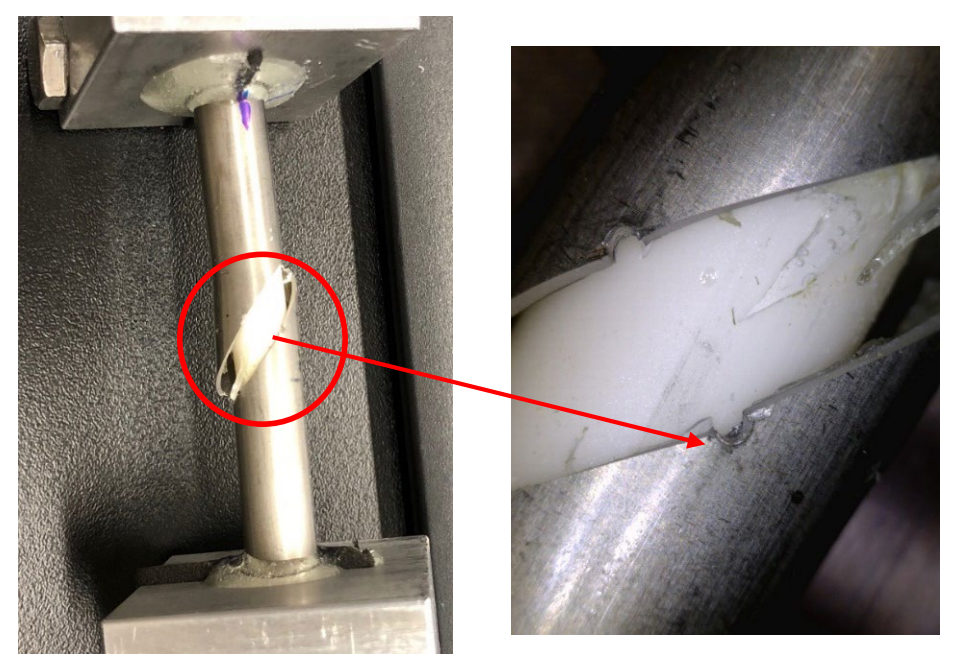

(b)

Fig. 36. (a) Fatigued failure pre-hydride Zr-4 clad SNTT sample with through thickness hole as crack starter, (b) which shows a brittle fracture characteristic w/o obvious fatigue pre-crack growth profile. 


\subsection{ZR-4 clad SNTT fracture testing procedures}

\subsubsection{Zr-4 clad SNTT sample cycle fatigue testing process}

The cycle fatigue process on Zr-4 clad SNTT samples were performed through the angle control mode by a function generator built in the TestResource control system. The selected initial fatigue cyclic loading is the torque that can reach about $60-80 \%$ of the maximum allowable shear stress level on the specimen surface. This is to ensure a linear elastic deformation existed during the fatigue cycling. In order to find the fatigue threshold of the Zr-4 clad SNTT samples, the initial maximum torque was adjusted to approximately $9.94 \mathrm{~N}-\mathrm{m}$ with $5 \mathrm{HZ}$ cyclic fatigue process. This cyclic load was gradually increased to facilitate the crack growth in a reasonably time frame to reach the targeted crack growth length ("a", notch initial length plus the fatigue crack growth length). The crack growth during the fatigue cycles was monitored by the specimen's compliance as well as periodically visual inspections. The target fatigue crack growth length in the axial direction is about $25 \%$ and $36 \%$ of pellet length for short and medium crack length, respectively; and the crack length in axial direction more than $60 \%$ is considered as a long crack length.

In order to introduce the mixed-mode loading into the pellet-clad system under pure torsion, the fatigue crack growth should reach sufficient length to activate higher pellet ends-clad pinning action (PCMI). All the fatigue crack growth at various lengths appear to be Mode-I fracture, where crack propagation orientation is along $45^{\circ}$ pitch angle, even with the longest fatigue crack growth sample. The final fracture mode was dictated by the length of the fatigue crack growth. From this study, for a long fatigue crack length, the final fracture contour showed a crack initiation profile of mixed-mode characteristic that is deviated from $45^{\circ}$ pitch angle of principal tensile stress; while as for medium and short crack length samples the final fracture contour is aligned with $45^{\circ}$ pitch angle of the principal tensile Mode I fracture.

\subsubsection{Zr-4 clad SNTT sample monotonic loading fracture test}

Fatigued SNTT sample was then loaded monotonically using the biaxial tester with series of loading/unloading sequences until failure; where the loading rate of $0.0056 \mathrm{~N}-\mathrm{m} / \mathrm{second}$ and unloading rate of $0.0113 \mathrm{~N}-\mathrm{m} / \mathrm{second}$ were used. The monotonic loading rates for ramping loading and unloading sequences were carried out under load control in each loading/unloading sequence to reach the target loading and the specified unloading range (normally about 10-15\% reduction in maximum load in each loading/unloading sequence). During the monotonic loading/unloading period, the axial force is maintained at nil zero condition to ensure a pure torsion loading condition. Upon final sudden fracture failure, a significant axial shock reaction force was observed, which was used to determine the final 
fracture torque load. The typical experimental test results for Zr-4 clad SNTT samples are shown in Fig. 37.
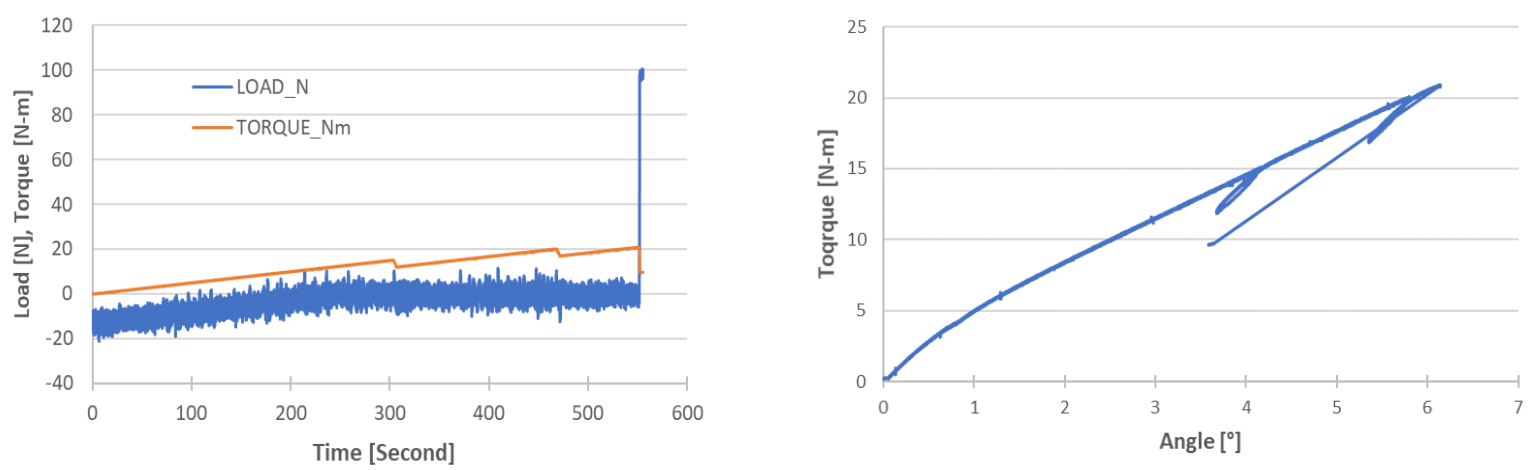

Fig. 37. Zr4-N3 sample test results, (Left) upon sudden failure a significant axial shock reaction force was observed, (Right) The slopes of different loading and unloading sequences does not change, indicate no crack growth during the monotonic loading, the specimen failed at $21 \mathrm{~N}-\mathrm{m}$ torque.

\subsubsection{Failed SNTT sample characterizations}

\subsubsection{SNTT specimens with medium fatigue pre-crack length}

The failed SNTT samples were characterized using an optical camera, which captured optical images of the specimens and the fractured surfaces, as shown in Fig. 38 for the tested SNTT samples with a $45^{\circ}$ notch crack starter and in Fig. 39 for the tested SNTT samples with a circular hole crack starter. Fig. 38a shows the three fracture profiles of SNTT samples with medium crack length, and Fig. 38b shows the detailed crack growth beyond the initial notch crack starter upon fracture initiation; where the fracture surface is normal to principal tensile stress profile.

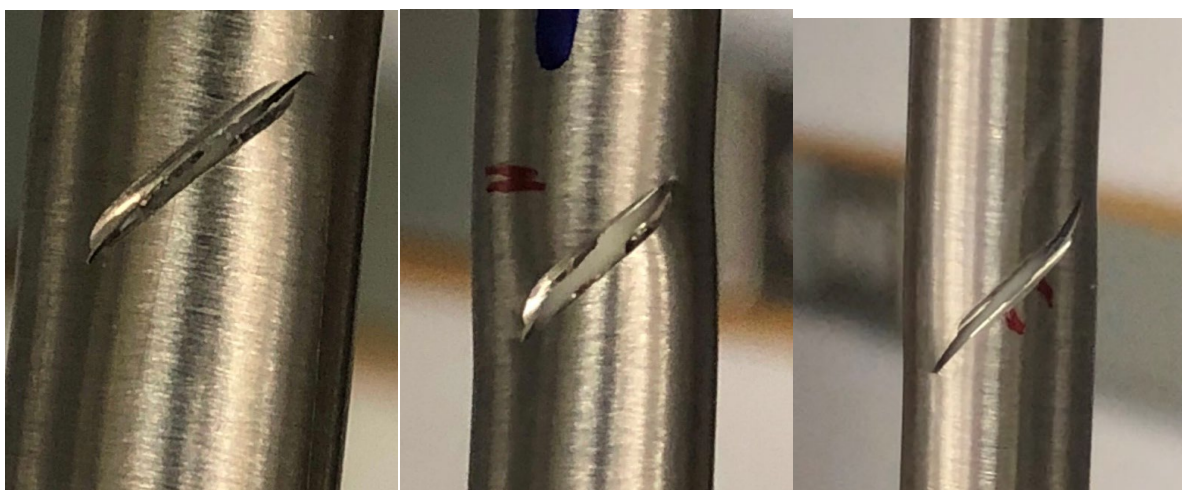

(a) 


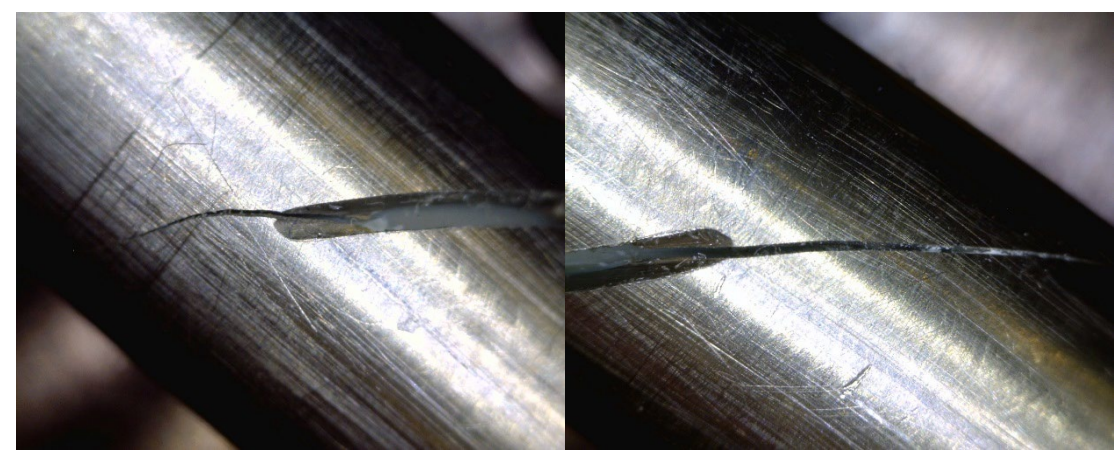

(b)

Fig. 38 (a) Three medium crack length Zr-4 clad SNTT samples fractured surface profiles, and (b) the detailed fracture surface profile beyond notch crack starter at fracture initiation.

Fig. 39 shows the fracture profiles of Zr-4 clad SNTT samples with a 0.762-mm diameter hole and shallow surface notch as crack starter. The fractured surfaces topology is aligned with a $45^{\circ}$ spiral crack fronts that is normal to the principal tensile stress profiles.
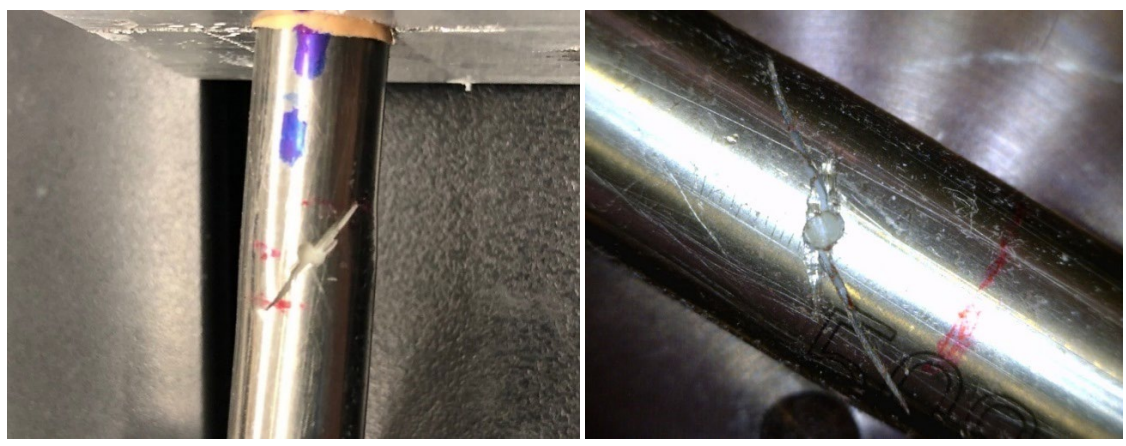

Fig. 39. Fractured Zr-4 clad SNTT samples of 9.525-mm diameter, (Left) a through thickness pin hole with shallow surface wing of 1.5- $\mathrm{mm}$ in length along $45^{\circ}$ slope as crack starter, (Right) a through thickness pin hole with shallow surface wing of 1.5- $\mathrm{mm}$ in length along $30^{\circ}$ slope as crack starter. Both types of the fatigued SNTT samples show that fatigued crack front and the final fracture profiles are aligned with the $45^{\circ}$ spiral crack front that is normal to the principal tensile stress contour profile.

\subsubsection{SNTT specimens with long fatigue pre-crack length}

The fractured SNTT Zr-4 N2B sample with long crack length revealed a mixed mode (Mode I + Mode III) failure mechanism as shown in Fig. 40, where the crack initiation orientation is deviated from the orientation normal to the tensile principal stress contour, i.e., along $45^{\circ}$ spiral crack front. 


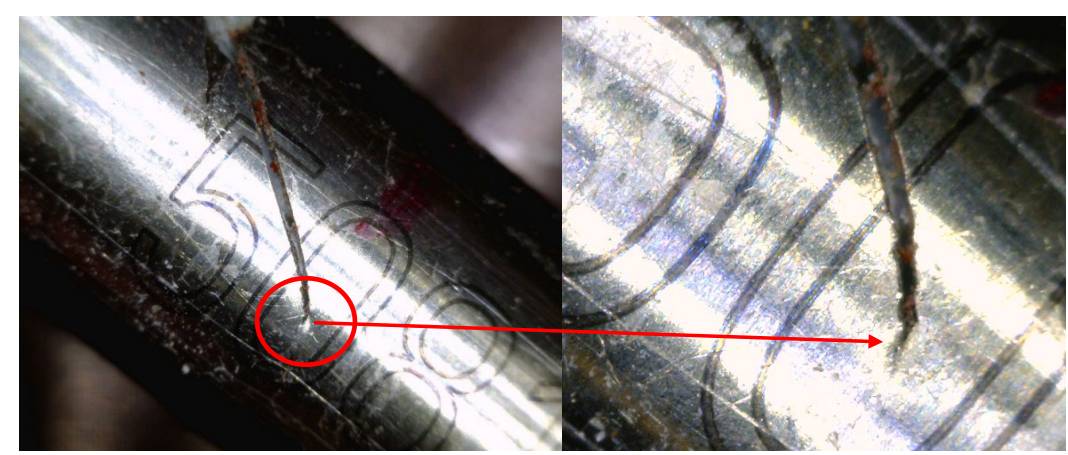

Fig. 40. (Left) Fracture profile of the Zr-4 clad SNTT specimen with long fatigue pre-crack length (Right) Detailed crack front view shows crack initiation direction is deviated from the $45^{\circ}$ spiral crack front, which indicates a mixed mode failure mechanism (Mode I + Mode III) under SNTT testing protocol.

The sources of the mixed-mode loading condition shown in Zr-4 N2B SNTT tested specimen failure profile are the combination of the SNTT pure torsion loading, and PCMI induced reaction forces between $15.2 \mathrm{~mm}$ pellet inserts and the clad tubing structure, including the pellet-pellet-clad interaction. For a long crack length, the out of plane shear load (Mode III) contributed from the pellet-pellet and pellet-clad pinning induced PCMI effect is expected to be significantly increased.

\subsubsection{Zr-4 clad SNTT samples fracture test results}

The details of the Zr-4 clad SNTT samples test results are illustrated in Table 4. Table 4 shows that the fracture torques from the different tests appear to be self-consistent at the targeted crack length, which indicates the good repeatability of the SNTT methodology in applying to the baseline ductile Zr-4 clad tubing materials.

Table 4 Summary of Zr-4 clad SNTT samples fracture test results

\begin{tabular}{llllll}
\hline Sample ID & $\begin{array}{l}\text { Projected crack } \\
\text { length in axial } \\
\text { direction, a(V) }\end{array}$ & $\begin{array}{l}\text { Total crack length } \\
\text { along surface } \\
\text { contour, a(T) }\end{array}$ & $\begin{array}{l}\mathrm{a}(\mathrm{V}) /(\text { pellet } \\
\text { length, } 15.24 \\
\mathrm{~mm}) \text { ratio }\end{array}$ & $\begin{array}{l}\mathrm{a}(\mathrm{T}) /(\text { Diameter, } \\
9.525 \mathrm{~mm}) \\
\text { ratio }\end{array}$ & $\begin{array}{l}\text { Fracture } \\
\text { Torque }\end{array}$ \\
\hline Zr4-n1 & $\mathrm{mm}$ & $\mathrm{mm}$ & 0.375 & 0.849 & $\mathrm{~N}-\mathrm{m}$ \\
Zr4-n2-A & 5.72 & 8.08 & 0.333 & 0.754 & 20.00 \\
Zr4-n2-B & 5.08 & 7.18 & 0.600 & 1.358 & 13.22 \\
Zr4-n3-B & 5.59 & 12.93 & 0.367 & 0.830 & 20.60 \\
Zr4-n4 & 3.89 & 7.90 & 0.255 & 0.577 & 22.99 \\
Zr4-n5 & 5.66 & 5.50 & 0.372 & 0.841 & 20.00 \\
Zr4-s1* & 5.59 & 8.01 & 0.367 & 0.830 & 20.99 \\
Zr4-s2* & 5.59 & 7.90 & 0.367 & 0.830 & 20.90 \\
\hline
\end{tabular}


*SNTT sample with a single alumina rod insert, instead of segment alumina pellet inserts.

\subsection{ZR-4 clad SNTT sample fracture toughness evaluation}

\subsubsection{Typical SNTT finite element modeling methodology for ductile materials}

\subsubsection{SNTT FEM solid model with surface crack flaw}

The methodology used for developing finite element model (FEM) and the typical FEM analyses results are illustrated in Fig. 5 [12]. The FEM designed for the ductile material SNTT fatigue pre-crack sample characterization was used for demonstration. For ductile material, the singular wedge element with quarter-node elements around crack tip was relaxed back to normal wedge element with middle-node elements. The typical FEM analyses results are also shown in Fig. 5 where the tri-axial tensile stress profiles and the butterfly plastic process zone indicate a high geometry constraint condition exists in the proposed SNTT fracture toughness testing protocol.

\subsubsection{SNTT finite element model for pellet-clad tubing structure with through clad thickness crack}

Two items of interest are considered for modeling PCMI, from the data obtained through CIRFT fatigue testing on SNF rods [32], namely, (1) the role of the pellet in nucleating fatigue failures (termed pelletcladding interaction) and (2) the extension or reduction of the fatigue lifetime resulting from mechanical or chemical bonding between the pellet and cladding or pellet and pellet (termed bonding). During reactor operation, pellet swelling and cladding creep down result in a mechanical interference fit between cladding and pellet that produces a mechanical bond through clad radial compressive residual stress. The mechanical bond is likely maintained throughout the fuel's dry storage and transport lifetime and is likely providing enhanced rod rigidity to SNF rod. There is evidence that a chemical bond between the zirconium-based fuel rod cladding and the uranium dioxide pellets can be developed at high burn-up, likely providing certain enhanced rod rigidity at chemically bonded locations. However, due to thermal expansion and contraction mismatch at clad-pellet interface region, during thermal cycling events of nuclear fuel operations, such a chemical bond is expected to be broken as shown from post-irradiation examination of mixed uranium-plutonium oxide SNF where the crack profile existed in the chemical bond region paralleled along the pellet-clad interface. Thus, the chemical bond effect on the SNF's enhanced rigidity is very limited.

The Abaqus J-integral contour routine was used in the J-integral value evaluation. The procedure of FEM approach used in $\mathrm{J}_{\mathrm{Q}}$ evaluation is stated as following: The FEM model was developed using FEM mesh generator, to match the SNTT sample geometry and the associated boundary conditions, such as the crack 
front profile, the total crack length, and SNTT sample geometry constraints. Final fracture torque obtained from SNTT experiment was used as load input in FEM model. In general, the unit rotation angle and the overall deformation obtained from the FEM results are quite consistent with that of SNTT experimental test results. The finite element model used for evaluating the apparent energy release rate, or $\mathrm{J}_{\mathrm{Q}}$, is shown in Fig. 41a, where 40,613 nodes and 9,362 3-D solid elements were used to model Zr-4 cladpellet system components. In order to simulate PCMI mechanism of SNF system with clad-pellet structure, the Abaqus "general contact algorithm," the hard normal contact mode with penalty functions and transverse shear criteria, was used in the FEM analyses; where the through-clad-thickness notch geometry and the associated crack fronts are shown in Fig. 41b.

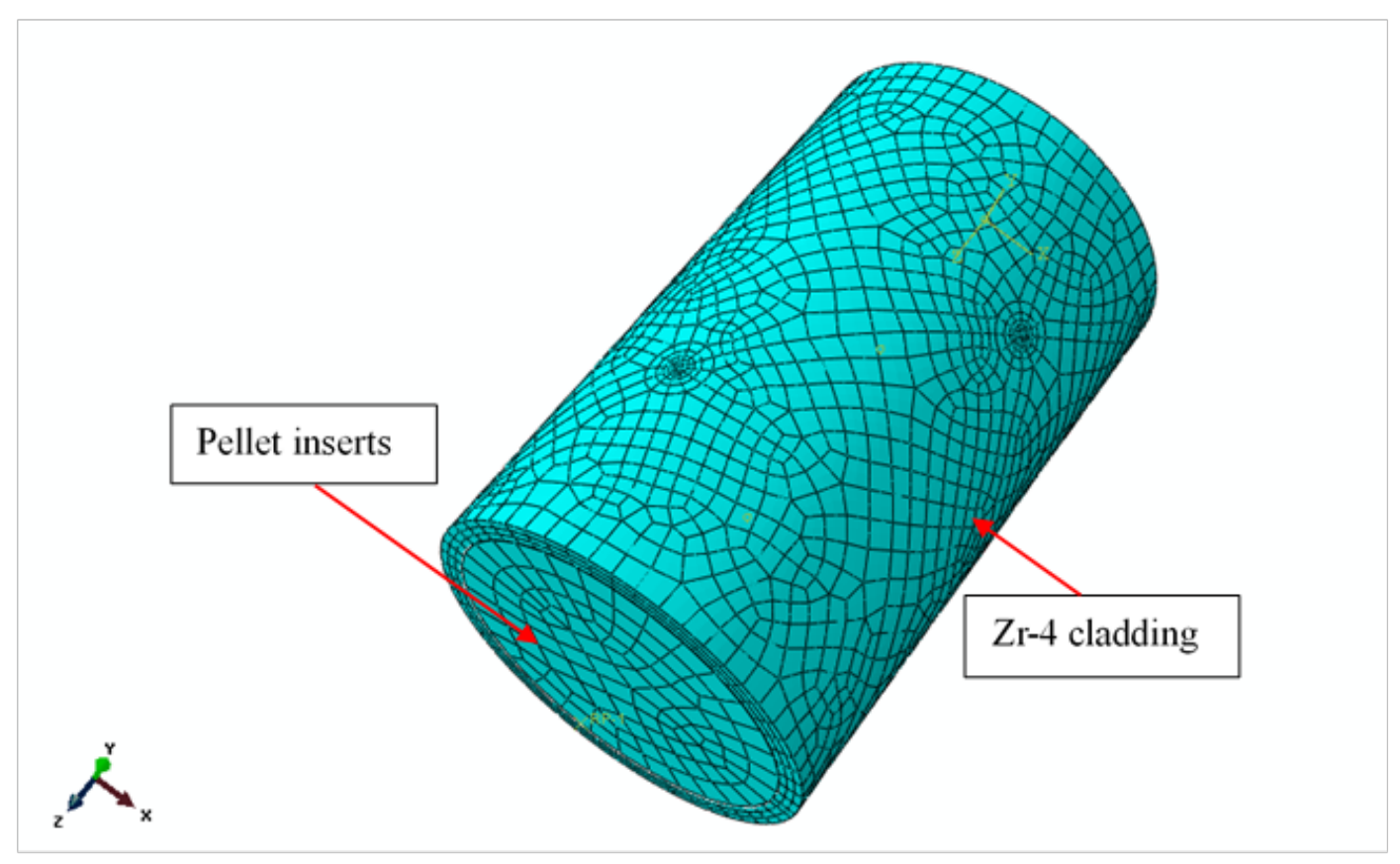

(a) 


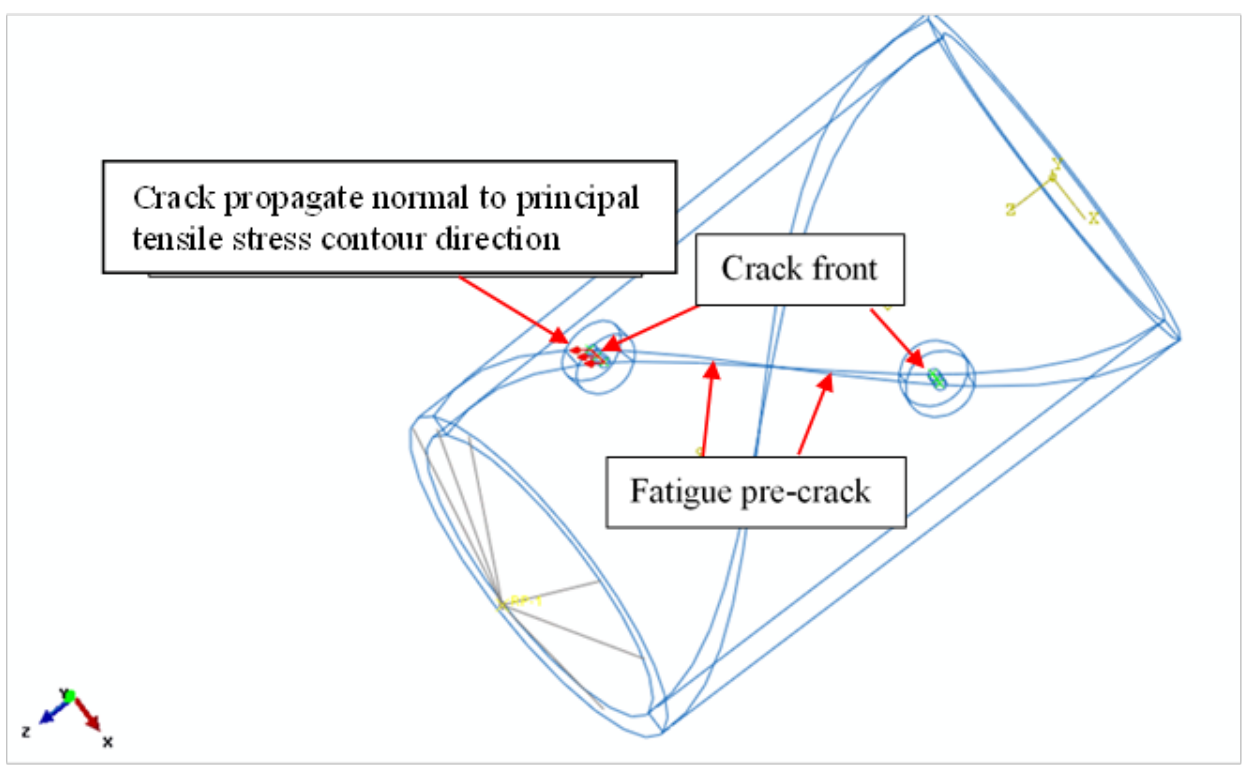

(b)

Fig. 41. (a) Clad-pellet FEM mesh profile, (b) Schematic diagram of crack seam and crack fronts profiles.

The details of mechanical properties, finite element model mesh and pellet-clad contact interaction criteria for Abaqus elastic-plastic analyses input are illustrated in Table 5 and Table 6.

Table 5 Zr-4 clad mechanical property for Abaqus elastic-plastic analyses input

\begin{tabular}{|c|c|c|c|c|}
\hline $\begin{array}{l}\text { Young's } \\
\text { modulus }\end{array}$ & $\begin{array}{c}\text { Yield } \\
\text { stress* }\end{array}$ & $\begin{array}{c}\text { Poisson's } \\
\text { ratio }\end{array}$ & $\begin{array}{l}\text { Plastic } \\
\text { strain }\end{array}$ & $\begin{array}{c}\text { True } \\
\text { stress }\end{array}$ \\
\hline $\mathrm{GPa}$ & $\mathrm{MPa}$ & & & $\mathrm{MPa}$ \\
\hline 91 & 606 & 0.33 & 0 & 574.5 \\
\hline \multirow{7}{*}{\multicolumn{2}{|c|}{ *0.2\% offset Yield stress }} & & 0.00125 & 604.7 \\
\hline & & & 0.00519 & 658.1 \\
\hline & & & 0.01074 & 713.1 \\
\hline & & & 0.01643 & 751.2 \\
\hline & & & 0.02818 & 785.3 \\
\hline & & & 0.03997 & 802.1 \\
\hline & & & 0.06321 & 828.7 \\
\hline
\end{tabular}


Table 6 FEM geometry, mesh, and clad-pellet contact criteria for Abaqus modeling input

\begin{tabular}{l}
\hline FEM mesh geometry \\
\hline Zr-4 clad: OD: $9.525-\mathrm{mm}$, Thickness: $0.57-\mathrm{mm}$, Length: $15.2-\mathrm{mm}$ \\
Pellet: OD: $8.33-\mathrm{mm}$, Length: $15.2-\mathrm{mm}$ \\
\hline Finite element type \\
\hline 3D Hex quadratic C3D20R reduced integration element \\
Around the crack front using 3D Wedge quadratic C3D15 element \\
\hline Mesh sizing control \\
\hline Approximate global size: 0.5 -mm \\
Curvature control: maximum deviation factor set 0.2 \\
Minimum size control: by fraction of global size of 0.2 \\
Minimum edge size for crack tip element: 0.05 -mm \\
\hline Pellet-clad mechanical interaction \\
\hline Normal Behavior: Hard contact, allow separation after contact \\
Tangential behavior: Penalty friction formulation algorithm with 0.3 friction coefficient \\
\hline Boundary condition \\
\hline Torque end: Kinematic coupling was used at Reference point to control the clad and pellet displacement \\
Reference point: Displacement and rotation fixed at X \& Y directions \\
Fixed end of clad and pellet insert: Displacement and rotation are fixed in X, Y, and Z directions \\
Torque load: Applied at reference point \\
\hline
\end{tabular}

\subsubsection{Energy release rate evaluation for Zr-4 clad SNTT sample with short and medium crack length}

\subsubsection{SNTT sample with short fatigue pre-crack length}

Totals of 42,311 nodes and 9,728 3-D solid elements were used to model Zr-4 clad component and for alumina pellet insert component with a crack length of $5.486 \mathrm{~mm}$ of SNTT sample Zr4-n4. The fracture torque is at $22.99 \mathrm{~N}-\mathrm{m}$. The deformed FEM model upon failure and the estimated von Miss stress contours are shown in Fig. 42. The Abaqus J-contour integral routine with 6-contours option was used to determine the $J$ value. Near middle layer's J-contour data were used to estimate $J_{Q}$ for $Z r 4 n 4$ specimen upon final fracture; which results in $\mathrm{J}_{\mathrm{IQ}}=50.79 \mathrm{~kJ} / \mathrm{m}^{2}$. 


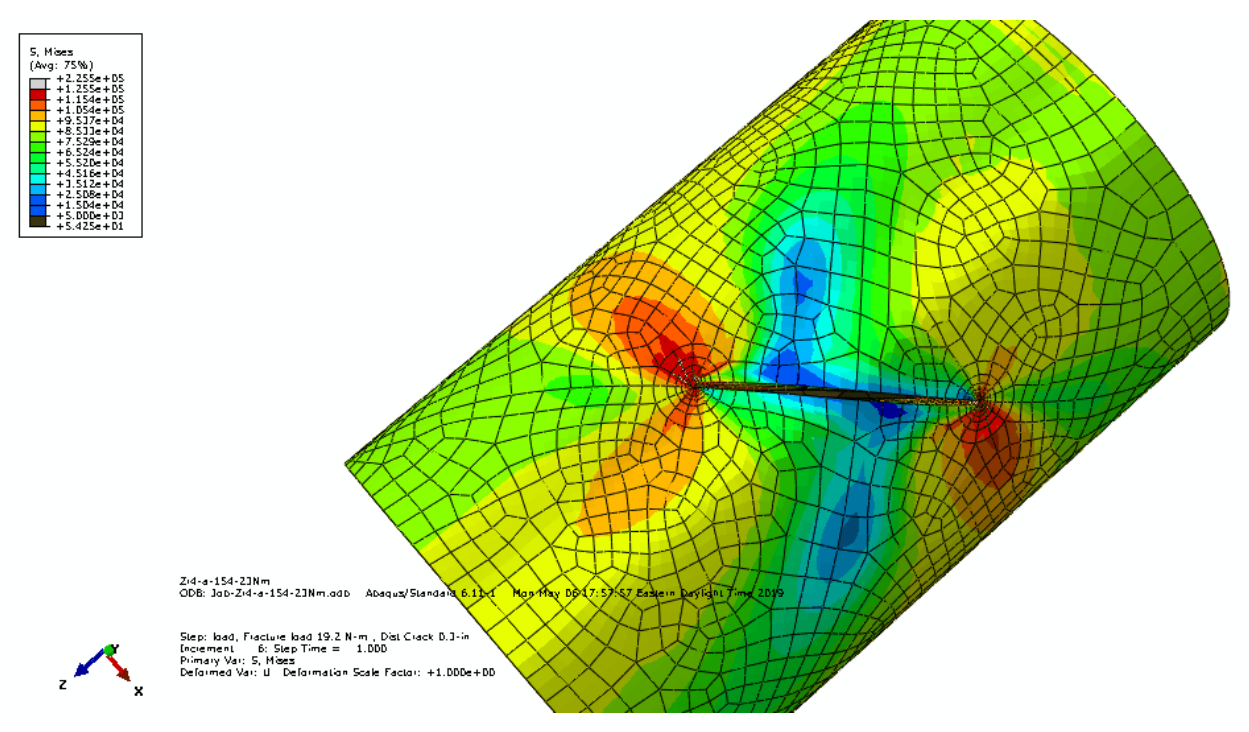

Fig. 42. FEM deformation of short crack SNTT sample and the associated von-Mises stress contours profile.

\subsubsection{SNTT sample with medium fatigue pre-crack length}

Totals of 40,613 nodes and 9,362 3-D solid elements were used to model Zr-4 clad and alumina pellet insert components with a crack length of $7.9 \mathrm{~mm}$ of SNTT sample Zr4-n3-B. The fracture torque is at 20 $\mathrm{N}-\mathrm{m}$. The deformed FEM model upon failure and the estimated von Miss stress contours are shown in Fig. 43. The Abaqus J-contour integral routine with 6-contours option was used to determine the J value. Near middle layer's J-contour data were used to estimate $\mathrm{J}_{\mathrm{Q}}$ for $\mathrm{Zr} 4-\mathrm{n} 3-\mathrm{B}$ specimen upon final fracture; which results in $\mathrm{J}_{\mathrm{IQ}}=49.38 \mathrm{~kJ} / \mathrm{m}^{2}$.

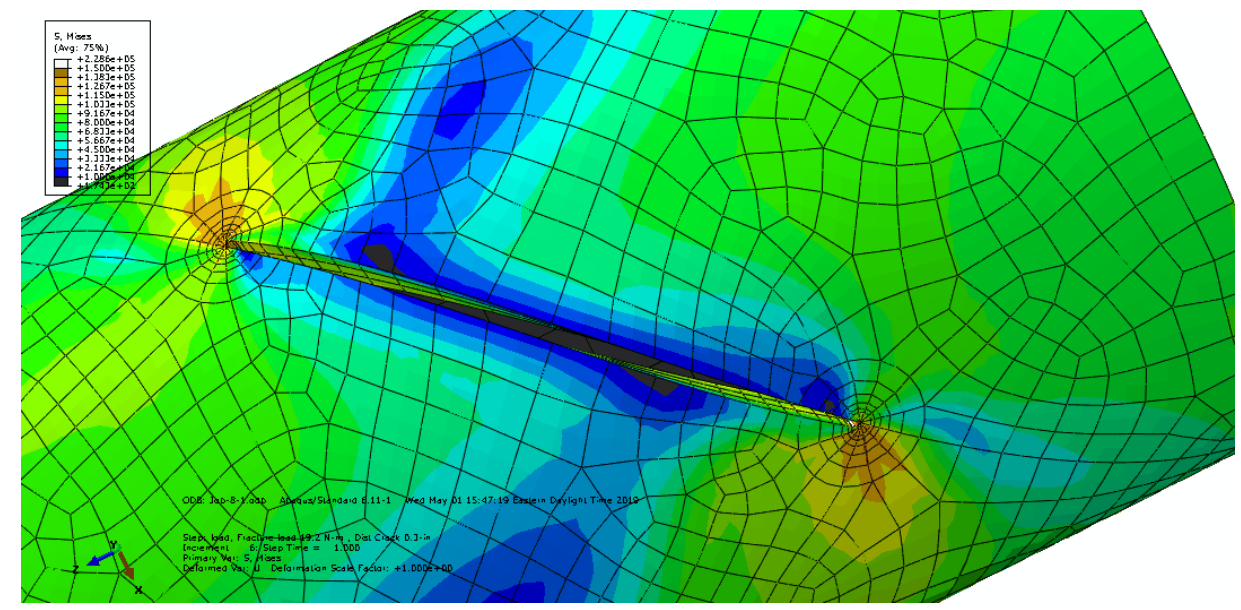

Fig. 43. FEM deformation of medium crack SNTT sample and the associated von Mises stress contours. 


\subsubsection{Energy release rate evaluation for Zr-4 clad SNTT sample with long crack length}

Totals of 40,613 nodes and 9,362-D solid reduced-integration elements were used to model $\mathrm{Zr}-4 \mathrm{clad}$ SNTT specimen with a crack length of $12.93-\mathrm{mm}$ of SNTT sample Zr4-n2-B. The fracture torque is at $13.19 \mathrm{~N}-\mathrm{m}$. The Abaqus J-contour integral routine with 6-contours option was used to determine J value. The deformed FEM model upon failure and the estimated von Mises stress contours are shown in Fig. 44. The un-symmetry von Mises stress contour was revealed in Fig. 43, in contrast to the symmetry butterflyshape of von-Mises stress contours near the crack tip region shown in Fig. 44. Furthermore, as shown in Fig. 40, the crack propagation direction is deviated from the principal tensile stress contour direction (i.e., in a mixed-modes, Mode I + Mode III, failure mechanism, instead of Mode I only failure mechanism). The estimated $\mathrm{J}_{\mathrm{MQ}}$ for $\mathrm{Zr} 4-\mathrm{n} 2-\mathrm{B}$ specimen upon final fracture is $18.91 \mathrm{~kJ} / \mathrm{m}^{2}$. The $\mathrm{J}$-value associated with the principal tensile stress contour orientation is at $35 \mathrm{~kJ} / \mathrm{m}^{2}$. The significant reduction in fracture toughness in association with mixed mode (Mode I + Mode III) loading compared to that of Mode I tensile loading alone for $\mathrm{Zr}-4$ tubing material deserved special attention. The similar behavior of the 50\% reduction in fracture toughness for ductile materials subjected to mixed-mode (Mode I + Mode III) loading compared to that of Mode I alone using the conventional compact tension specimen approach was also reported in Reference 27.
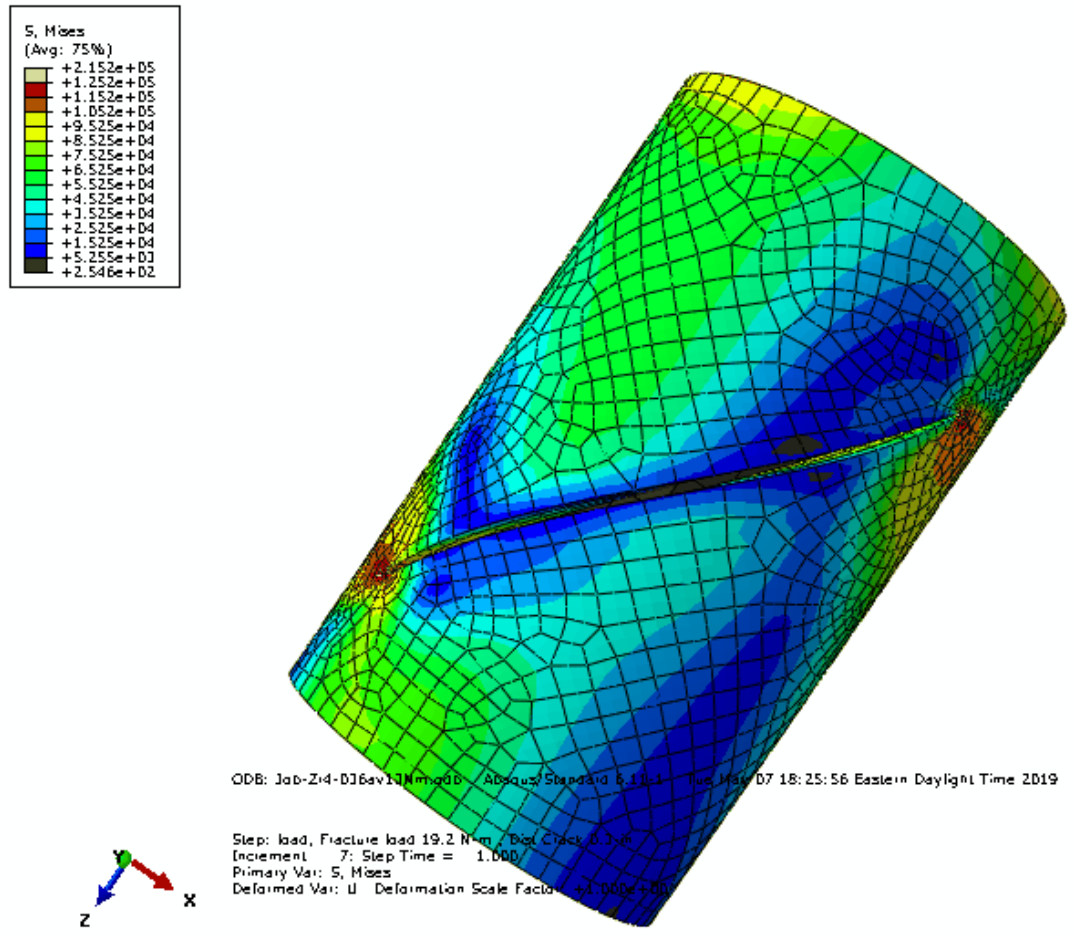

(a) 


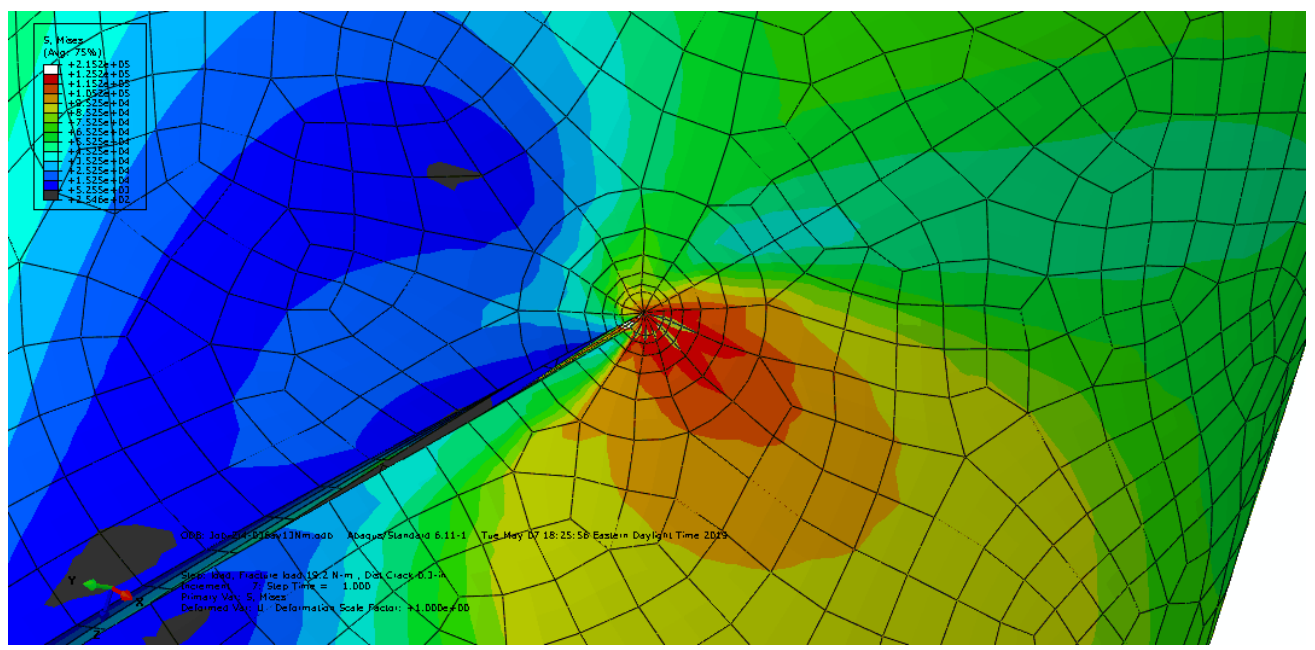

(b)

Fig. 44. (a) FEM deformation of long crack SNTT sample and associated von-Mises stress contours profile; (b) The detailed von Miss stress contour view at crack tip.

\subsubsection{Non-coplanar crack propagation orientation}

In many practical situations structures are subjected to a combination of both shear and tensile/compression loading, especially for a tubing or piping structure, leading to a mixed-mode fracture. The strain energy density criterion [33] states that crack growth takes place in the direction of minimum strain energy density factor $S$. The 3-D energy density factor can be written [34] as

$$
S=a_{11} K_{l}^{2}+2 a_{12} K_{l} K_{I I}+a_{22} K_{l l}^{2}+a_{33} K_{l I I}^{2}
$$

Where $K_{i}$ is referred to stress intensity factors, and $a_{i j}$ is the function of shear modulus, Poisson ratio, and the projected space angle $\theta$. The crack propagation orientation is referred to at a critical angle, $\theta_{0}$, the associated $S$ has the minimum value, $S_{\text {cr. }}$ Since $S$ is proportional or scaled to the J-value, thus, the crack propagation orientation in a mixed-mode loading condition is also referred to a critical angle that has minimum $\mathrm{J}$ value, $\mathrm{J}_{\mathrm{Q}}$.

\subsubsection{Fracture toughness evaluation of the tested Zr-4 clad SNTT samples}

The Zr-4 clad SNTT fracture test data and the associated fracture toughness (represented with J-integral value at final fracture load, $\mathrm{J}_{\mathrm{Q}}$ ) are illustrated in Table 7, for samples with short and medium crack lengths. Small two-sigma uncertainty bond observed from SNTT tests is primary due to the self- 
consistent fracture torques observed from the SNTT fracture test results as shown in Table 7. The mean $\mathrm{J}_{0.2}$ obtained for $\mathrm{Zr}-4$ clad tubing from double edge notched tension (DENT) specimens, using wedge inserts induced clad lateral expansion approach, is at $70.5 \pm 6.4 \mathrm{~kJ} / \mathrm{m}^{2}$ [35]. This is about $40 \%$ larger than that of SNTT approach at $50 \mathrm{~kJ} / \mathrm{m}^{2}$. The root cause of larger J-value obtained from DENT test results could be primary due to significant shear forces induced from contact interactions between the two-wedge inserts and the clad tubing inner wall, in addition to the potential clad bending deformation along the edge of wedges under clad later expansion loading. The consequence of the observed fatigued crack fronts deviated from the initial axial crack orientation from DENT tested sample [35], can also induced mixed mode failure mechanism of Mode I and Mode II (in-plane shear) on the clad specimen.

Table 7 Summary of fracture toughness obtained from SNTT tests with medium crack length

\begin{tabular}{llllll}
\hline Sample ID & $\begin{array}{l}\text { Crack length } \\
\text { projected in axial } \\
\text { orientation, } \mathrm{a}(\mathrm{V})\end{array}$ & $\begin{array}{l}\text { Total crack } \\
\text { length } \\
\mathrm{a}(\mathrm{T})\end{array}$ & Fracture Torque & $\mathrm{J}_{\mathrm{IQ}}$ & $\begin{array}{l}\mathrm{K} \text { IQ } \\
=\sqrt{\left(\mathrm{E}^{*} \mathrm{~J}\right)}\end{array}$ \\
\hline $\mathrm{mm}$ & $\mathrm{mm}$ & $\mathrm{N}-\mathrm{m}$ & $\mathrm{kJ} / \mathrm{m}^{2}$ & $\mathrm{MPa} \sqrt{\mathrm{m}}$ \\
\hline Zr4-n1 & 5.72 & 8.08 & 19.21 & 46.62 & 65.13 \\
Zr4-n2-A & 5.08 & 7.18 & 20.00 & 44.92 & 63.93 \\
Zr4-n2-B & 9.14 & 12.93 & 13.22 & 35.03 & 56.45 \\
Zr4-n3 & 5.59 & 7.90 & 20.60 & 49.41 & 67.05 \\
Zr4-n4 & 3.89 & 5.50 & 22.99 & 50.79 & 67.98 \\
Zr4-n5 & 5.66 & 8.01 & 20.00 & 50.09 & 67.51 \\
Zr4-s1* & 5.59 & 7.90 & 20.99 & 54.47 & 70.40 \\
\hline & & & Average & 50.04 & 67.44 \\
& & & 2 -sigma bound & 3.26 & 2.20 \\
\hline
\end{tabular}

*SNTT sample with a single alumina rod insert, instead of segment alumina pellet inserts.

The J-integral values along fracture plane and at other orientations for Zr4-n2-B specimen with long crack length are illustrated in Table 8; where mixed-mode fracture toughness of $18.91 \mathrm{~kJ} / \mathrm{m}^{2}$ shows a significant reduction compared to that of Mode I alone toughness at $35.03 \mathrm{~kJ} / \mathrm{m}^{2}$. The similar behavior of the $50 \%$ reduction in fracture toughness for ductile materials subjected to mixed-mode (Mode I + Mode III) loading compared to that of Mode I alone using the conventional compact tension specimen approach was also reported in Reference 27. 
Table 8 Summary of mixed-mode fracture toughness obtained from SNTT tests with long crack length

\begin{tabular}{|c|c|c|c|c|c|c|}
\hline Sample ID & $\begin{array}{l}\text { Total crack } \\
\text { length, } a(T)\end{array}$ & $\begin{array}{l}\text { Fracture } \\
\text { Torque }\end{array}$ & $\begin{array}{l}\text { Crack front propagation } \\
\text { orientation or projected } \mathrm{J}- \\
\text { contour orientation }\end{array}$ & Loading Modes & $\mathrm{J}_{\mathrm{Q}}$ & $\begin{array}{l}\mathrm{K}_{\mathrm{Q}} \\
=\sqrt{ }_{\left(\mathrm{E}^{*} \mathrm{~J}\right)}\end{array}$ \\
\hline \multirow{4}{*}{$\mathrm{Zr} 4-\mathrm{n} 2-\mathrm{B}$} & $\mathrm{mm}$ & $\mathrm{N}-\mathrm{m}$ & q-vector & & $\mathrm{kJ} / \mathrm{m}^{2}$ & $\mathrm{MPa} \sqrt{\mathrm{m}}$ \\
\hline & \multirow{3}{*}{12.92} & \multirow{3}{*}{13.22} & $\begin{array}{l}\text { Along final fracture surface } \\
\text { contour }\end{array}$ & $\begin{array}{l}\text { Mode I + Mode III - } \\
\text { (out of plane shear) }\end{array}$ & 18.91 & 41.53 \\
\hline & & & $\begin{array}{l}\text { Perpendicular to the principal } \\
\text { tensile stress }\end{array}$ & Mode I (tensile) & 35.03 & 56.47 \\
\hline & & & $\begin{array}{l}\text { Between fracture surface } \\
\text { contour and the principal } \\
\text { tensile stress contour profiles }\end{array}$ & Mode I + Mode III & 29.07 & 51.42 \\
\hline
\end{tabular}




\section{CONCLUSIONS}

SNTT method has been developed for estimating the opening mode fracture toughness, $K_{I C}$ or $J_{I C}$. A round-bar specimen having a spiral $V$-groove line at $45^{\circ}$ pitch angle is used, subjected to pure torsion. The other unique feature of the proposed testing method is fracture failure in combined mixed-mode (Mode I and Mode III) pertinent to piping or clad tubing systems can be tailored for simulation study by varying the pitch angle of the starting notch line deviated from $45^{\circ}$ pitch angle or alternatively having the SNTT standard specimen subjected to various combinations of loads in tension and torsion.

The unique features of the SNTT method are:

- It conforms to the classical theory of fracture mechanics.

- It is not limited by sample size or volume.

- It controls crack propagation and thus produces consistent results.

- It can test a wide variety of materials, such as metals and alloys (including thin film coating and interface material), ceramics, composites, polymers, and concrete.

A new J-integral evaluation protocol was developed based on the measured fracture torque and torque ends rotation angle trend curve, obtained from the SNTT method. The estimated SNTT fracture toughness $\mathrm{J}_{\mathrm{Q}}$, for the SS304/308 weldment is in consistent with that using conventional CT specimens. The SNTT test results indicate that SNTT method is a reliable test approach with good repeatability in applying to $\mathrm{SS} 304 / 308$ weld material. The estimate $\mathrm{J}_{\mathrm{Q}}$ ' upon fracture for the baseline SS304 steel is at $459 \mathrm{~kJ} / \mathrm{m}^{2}$. The estimate $\mathrm{J}_{\mathrm{Q}}$, upon fracture for the $\mathrm{SS} 304 / 308$ weld from weld specimens is $144.2 \mathrm{~kJ} / \mathrm{m}^{2}$.

In this project, we also have successfully extended SNTT approach to a highly ductile Zr-4 thin-wall clad tubing structure with pellets inserts, the estimated $\mathrm{J}_{\mathrm{IQ}}$ value for the short and medium crack length is at 50 $\mathrm{kJ} / \mathrm{m}^{2}$. The clad-pellet structure mixed-mode toughness study was originated from the observation of the final fracture initiation contour pitch angle change, deviated from the principal tensile stress contour of $45^{\circ}$ pitch angle, from a long fatigue pre-crack SNTT sample. A significant reduction in fracture toughness for clad tubing structure under mixed-mode loading, Mode I (tension) and Mode III (out of plane shear), compared to that of Mode I only was revealed from tested Zr-4 clad SNTT specimen with long crack length. Furthermore, a significant reduction in ductility was also observed from the pre-hydride Zr-4 clad SNTT samples. This mixed-mode fracture results in a significant reduction in the J-integral value. Further parameters study, such as pellet dimensions, is warranted for clad-pellet structure mixed-mode fracture behavior study for the consideration related to the pellet-clad interaction induced PCMI effect. 


\section{REFERENCE}

1. D.G. Enos and C.R. Bryan, Final Report: Characterization of Canister Mockup Weld Residual Stress. Sandia National Laboratory, November 22, 2016. FCRD-UFD-2016-000064.

2. J.A. Wang, A. Payzant, J. Bunn, K. An, Neutron residual stress mapping for spent nuclear fuel storage canister weldment, 2018, ORNL/TM-2018/827, 2018.

3. Stainless Steels Properties - How to weld them and where to use them. The Lincoln Electric Company.

http://www.lincolnelectric.com/assets/global/Products/Consumable_StainlessNickelandHighAlloyExcalibur- Excalibur316316L-17/c64000.pdf

4. http://www.lincolnelectric.com/assets/global/Products/Consumable_StainlessNickelandHighAlloyLincolnweld-Lincolnweld308308L/c61024.pdf.

5. J.R. Rice, Mathematical analysis in the mechanics of fracture, An Advanced Trestise, Vol II, 191308, H. Liebowitz, editor, Academic Press, N. Y., 1968.

6. J.A. Begley and J.D. Landes, The J-Integral as a fracture criterion, Fracture Toughness, ASTM STP 514, 1-23, American Society for Testing and Materials, 1972.

7. J.W. Hutchinson and P.C. Paris, Stability analysis of J-controlled crack growth, Elastic-Plastic Fracture, ASTM STP 668, 37-64, American Society for Testing and Materials, 1979.

8. G. Bertolino, J. Perez Ipina, G. Meyer, Influence of the crack-tip hydride concentration on the fracture toughness of zircaloy-4. J. Nucl. Mater., 348 (2006) 205-212.

9. R.S. Daum, Hydride Induced Embrittlement of Zircaloy-4 Under Plane Strain Tension Testing. $\mathrm{PhD}$ Thesis in Materials Science, Penn State University, 2007.

10. H.H. Hsu, L.W. Tsay, Effect of hydride orientation on fracture toughness of Zircaloy-4 cladding. Nucl. Mater., 408 (2011) 67-72.

11. P.A. Raynaud, D.A. Koss, A.T. Motta, and K.S. Chan. Fracture toughness of hydrided zircaloy-4 sheet under through-thickness crack growth conditions. Journal of ASTM International, 5 (2008) 1, $1-15$.

12. J.A. Wang, K. Liu, D. McCabe, S. David, Using torsional bar testing to determine fracture toughness, Fatigue Fract Engng Mater Struct 23 (2000) 917-927.

13. J.A. Wang, K.C. Liu, A new approach to evaluate fracture toughness of structural materials. Journal of Pressure Vessel Technology, 126 (2004) 534-540.

14. J.A. Wang, I.G. Wright, M.J. Lance, K..C Liu, A new approach for evaluating thin film interface fracture toughness, Journal of Materials Science and Engineering A, 426 (2006) 332-345.

15. J.A. Wang, K. Liu, An innovative technique for evaluating fracture toughness of graphite materials. Journal of Nuclear Materials, 381 (2008) 77-184.

16. J.A. Wang, K. Liu, D. Naus, A new test method for determining the fracture toughness of concrete materials. Journal of Cement and Concrete Research, 40 (2010) 497-499.

17. T. Tan, F. Ren, J.A. Wang, E. Lara-Curzio, P. Agastra, J. Mandell, et al. Investigating fracture behavior of polymer and polymeric composite materials using spiral notch torsion test. Journal of Engineering Fracture Mechanics, 10 (2013) 109-128.

18. F. Ren, J.A. Wang, W. Bertelsen, Fractographic study of epoxy materials fractured under mode I loading and mixed mode I/III loading. Materials Science and Engineering A, 532 (2012) 449-455.

19. J.A. Wang, Fracture toughness evaluation for thin-shell stainless steel weldment, Journal of 
Theoretical and Applied Fracture Mechanics, 106 (2020), 102467. https://doi.org/10.1016/j.tafmec.2019.102467

20. J.A. Wang, F. Ren, T. Tan, K. Liu, The development of in situ fracture toughness evaluation techniques in hydrogen environment, International Journal of Hydrogen Energy, 40 (2015) 20132024.

21. J.A. Wang, T. Tan, A method for evaluating the fatigue crack growth in spiral notch torsion fracture toughness test. Archived of Applied Mechanics Journal, 89 (2019) 813-822.

22. J. R. Rice, "A path-independent integral and the approximate Analysis of strain concentration by notches and cracks,” Journal of Applied Mechanics, Trans. ASME, 35 (1968) 379-386.

23. G.P. Cherapnov, "Crack propagation in continuous media," Applied Mathematics and Mechanics, (trans. P.M.M.), 31 (1967) 476-488.

24. (26) J.R. Rice, P.C. Paris, J.G. Merkle, Some further results of J-integral analysis and estimates. Progress in flaw growth and fracture toughness testing, ASTM STP 536, 231-245, American Society for Testing and Materials, 1973.

25. Mills WJ, Fracture toughness of type 304 and 316 stainless steels and their welds. International Materials Reviews, 42:2 (1997) 45-82, DOI: 10.1179/imr.1997.42.2.45.

26. K. C. Liu and J. A. Wang, An energy method for predicting fatigue life, crack orientation, and crack growth under multiaxial loading conditions. International Journal of Fatigue, 23 (2001), 129134.

27. H.X. Li, R.H. Jones, J.P. Hirth, D.S. Gelles, Fracture toughness of the F-82H steel-effect of loading modes, hydrogen, and temperature. J Nucl Mater 1998;233(0):258-63.

28. M.R.M. Aliha, A. Bahmani, F. Berto, S. Akhondi, A. Barnoush, Fracture assessment of polymethyl methacrylate using sharp notched disk bend specimens under mixed-mode I + III loading. Physical Mesomechanics, 19 (2016) 4 355-364.

29. A. Bahmani, M.R.M. Aliha, F. Berto, Investigation of fracture toughness for a polycrystalline graphite under combined tensile-tear deformation. Theoretical and Applied Fracture Mechanics 90 (2017) 53-64.

30. M.R.M.Alihaa, A.Razmib, A.Mousavia, Fracture study of concrete composites with synthetic fibers additive under modes I and III using ENDB specimen. Construction and Building Materials, 190 (2018) 30 612-622.

31. M.R.M. Aliha, A. Bahmani, S. Akhondi, Numerical analysis of a new mixed mode I/III fracture test specimen. Engineering Fracture Mechanics 134 (2015) 95-110.

32. J.A. Wang, H. Wang, H. Jiang, B. Bevard, High burn-up spent nuclear fuel transport reliability investigation. Nuclear Engineering and Design Journal, 330 (2018) 497-515.

33. I.S. Sokolnikoff, (1956), Mathematical Theory of Elasticity, 2nd edn. McGraw-Hill, New York, NY, USA, pp. 318-327.

34. R.J. Hartranft, G.C. Sih, Stress singularity for a crack with an arbitrary curved front. Engineering Fracture Mechanics, 9 (1977), 705-718.

35. J. Bertsch, W. Hoffelner, Crack resistance curves determination of tube cladding material. Nucl. Mater., 352 (2006) 116-125. 
APPENDIX A: TESTRESOURCE ELECTRODYNAMIC SYSTEM USER MANUAL 


\title{
TESTRESỌURCES
}

\section{Electrodynamic System User Manual}

\author{
Installation, Operation, \& MaINTENANCE
}

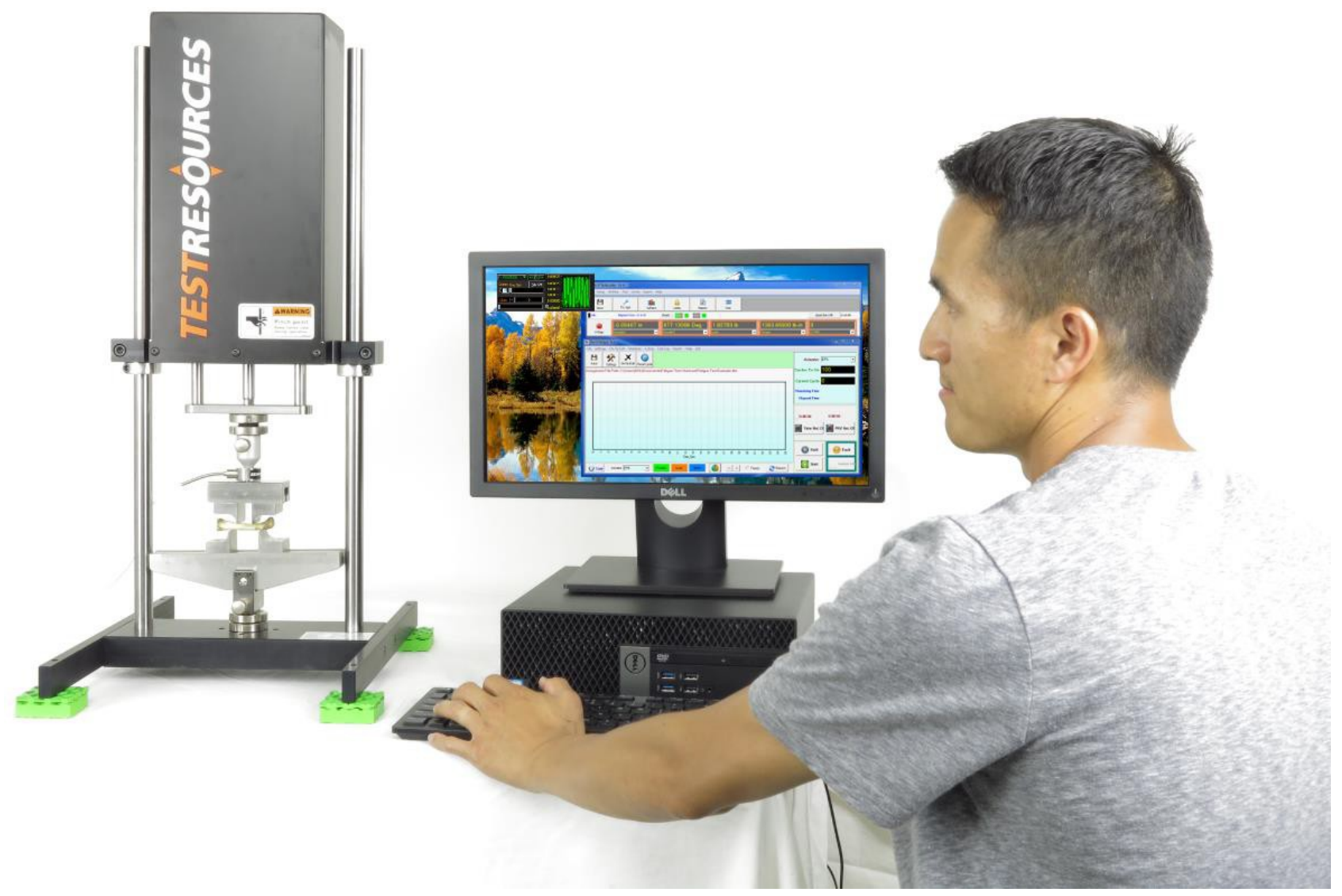

Edition: September 2016

Valid for all Electrodynamic

Testing Systems Part Number

81000001-01

Original Document 
[This Page is Intentionally Left Blank] 


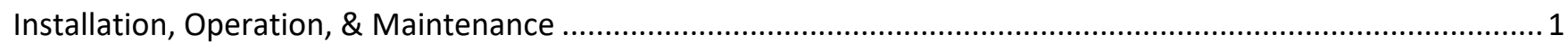

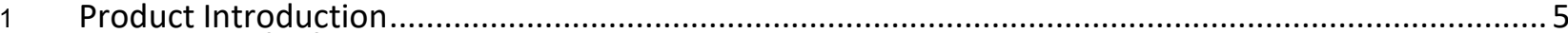

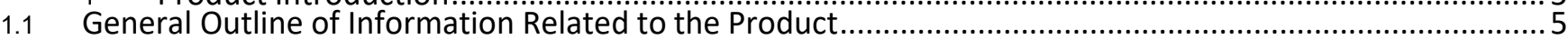

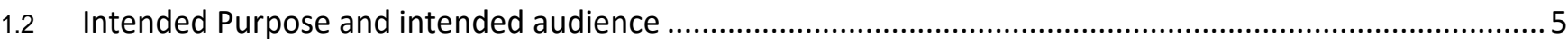

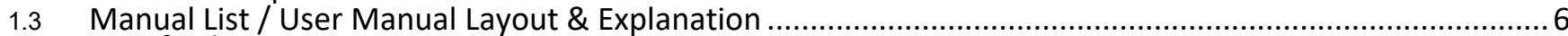

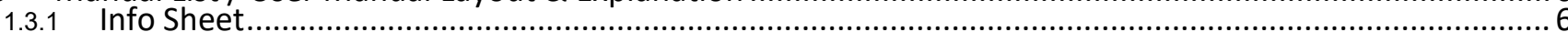

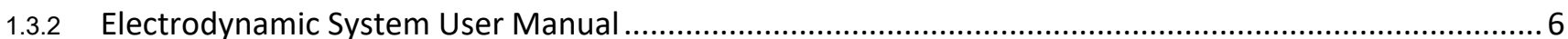

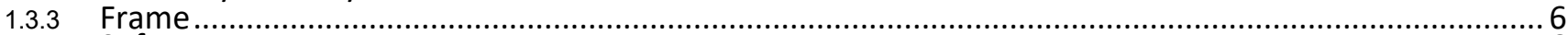

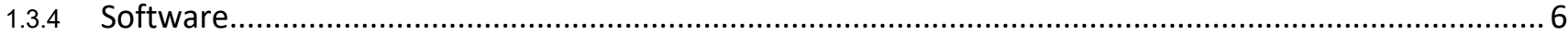

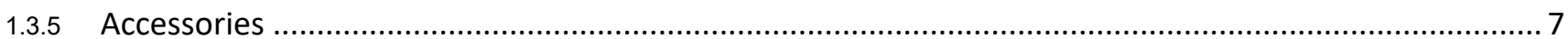

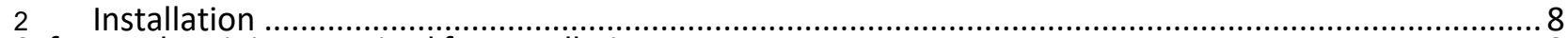

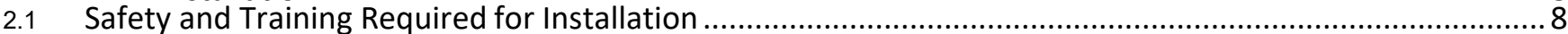

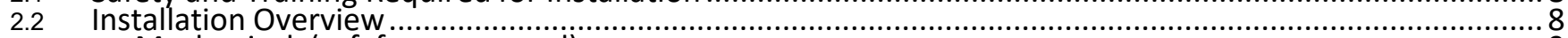

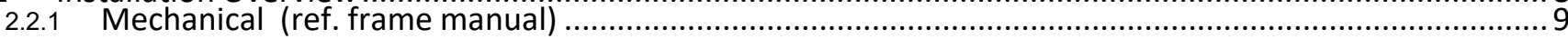

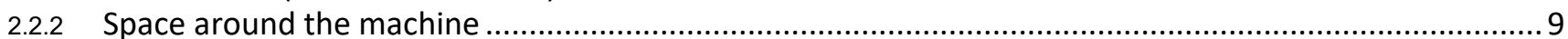

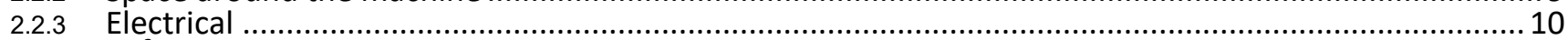

2.2.4 Software

2.3 Additional required items needed to support the testing system .....................................................13

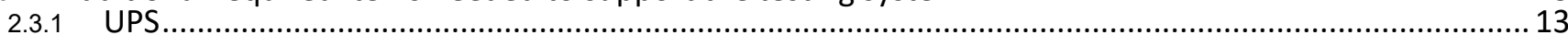

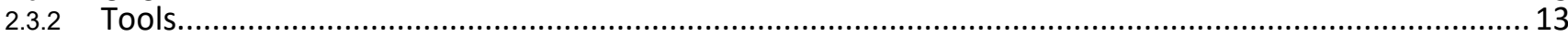

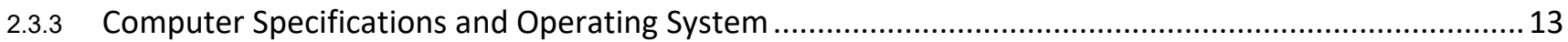

$2.4 \quad$ Additional items that support the system, but are not required ..................................................... 13

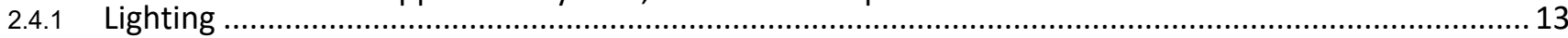

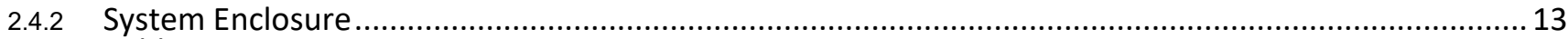

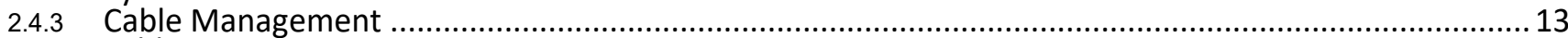

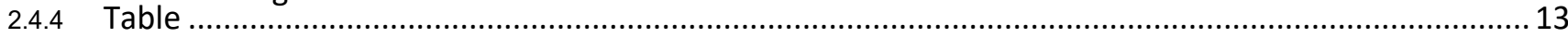

2.4.5 Storage

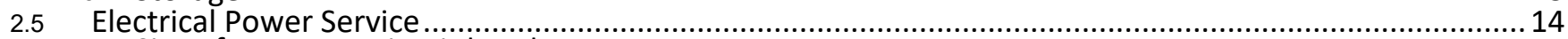

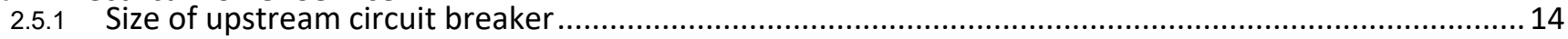

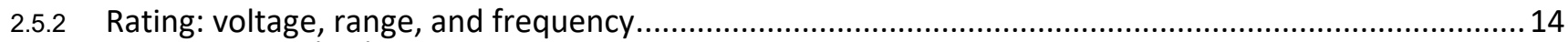

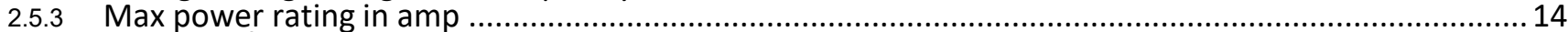

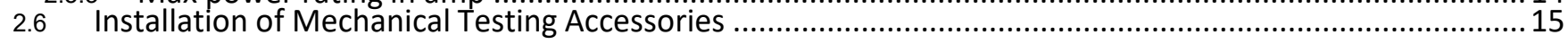

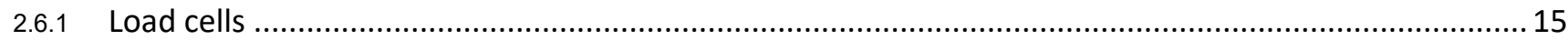

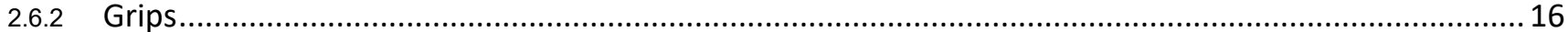

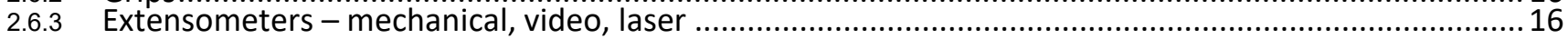

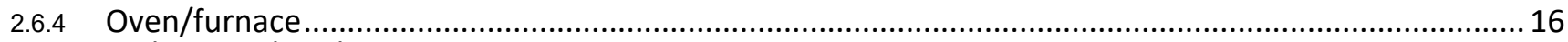

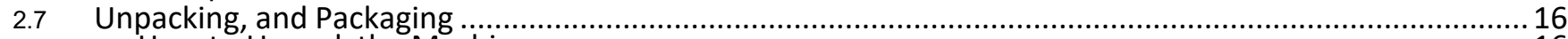

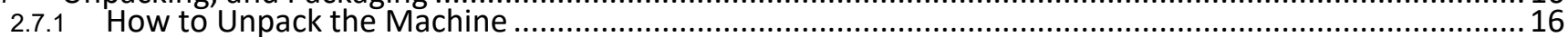

$2.8 \quad$ Final review of check-off/inspection for a correct installation.......................................................... 16

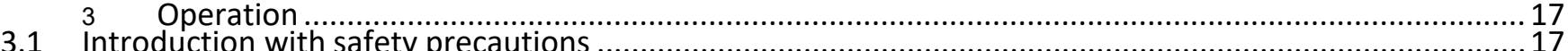

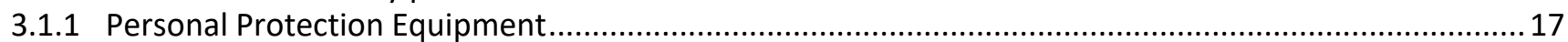

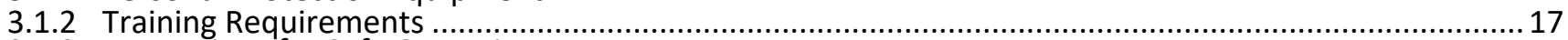

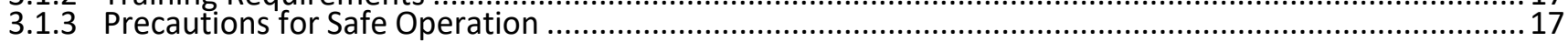

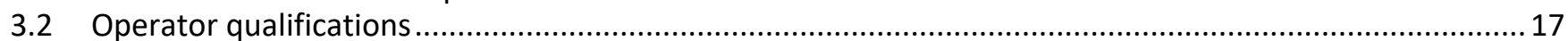

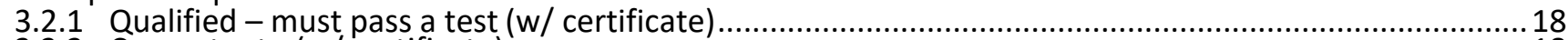

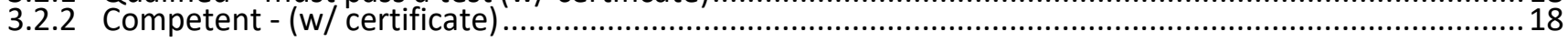

3.3 Description/explanation of each hazard warning label................................................................... 18

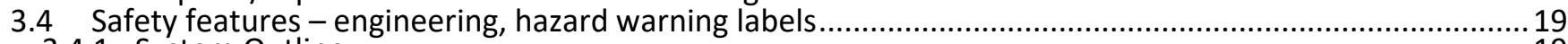

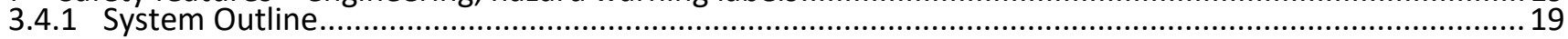

3.5 Equipment - describes set-up, equipment use, programing, etc...............................................................2 21

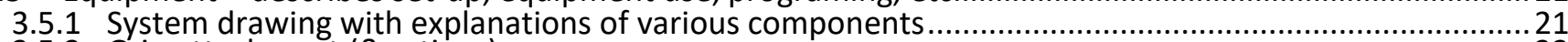

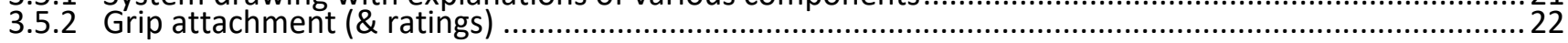

3.5.3 Basic test setup and operation - quick start. Reference s/w manuals as needed .............................22

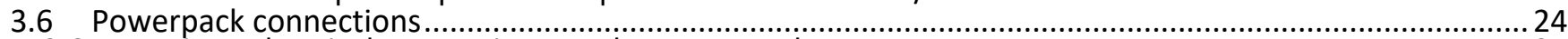

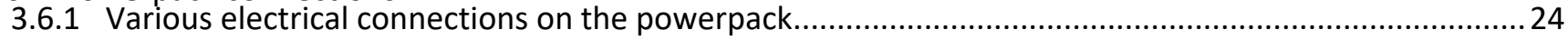

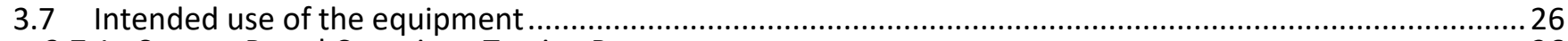

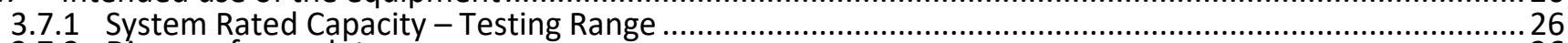

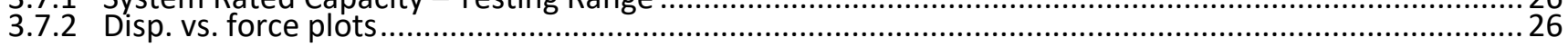

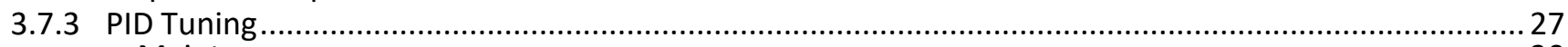

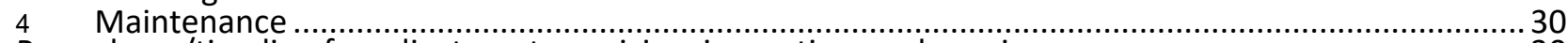

4.1 Procedures/timeline for adjustment, servicing, inspection, and repair.......................................................30

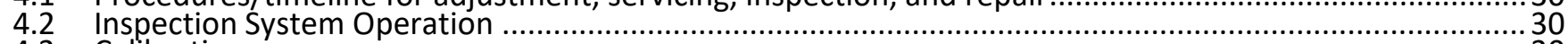

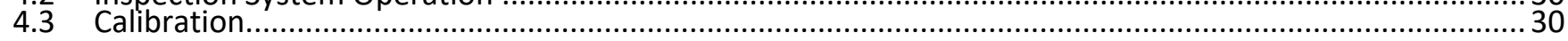

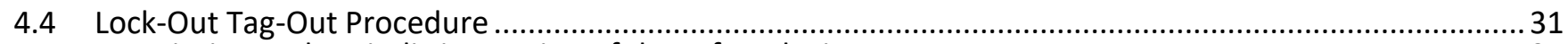

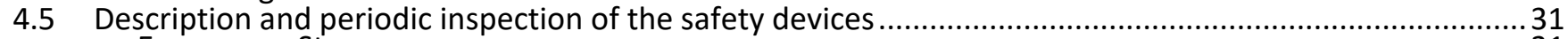

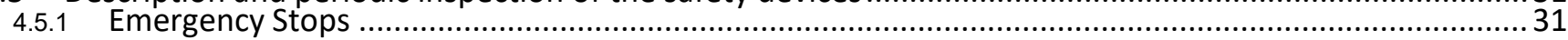

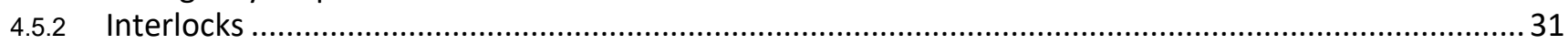


4.5.3 Panel LED's.

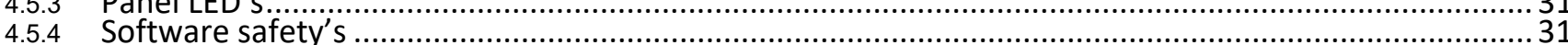

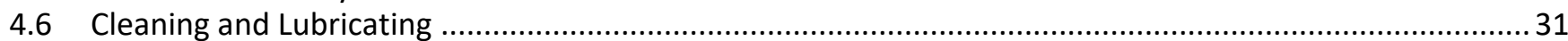

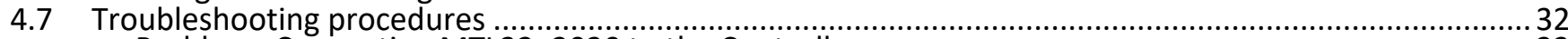

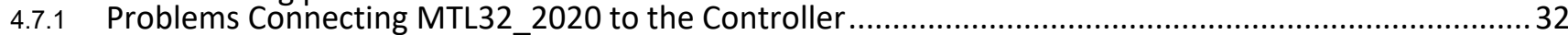

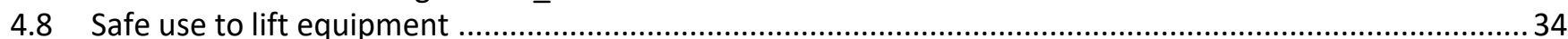

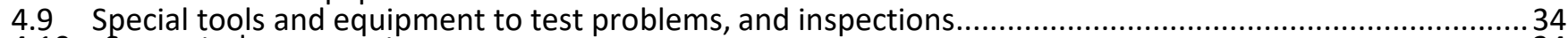

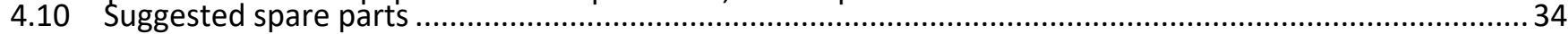

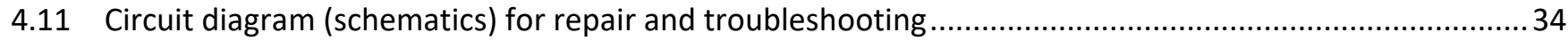

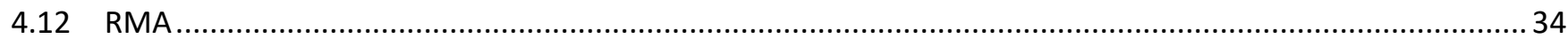


Questions on the operation of the machine may be answered by contacting TestResources directly at the following: Technical Support (8:00 - 5:00 CT)

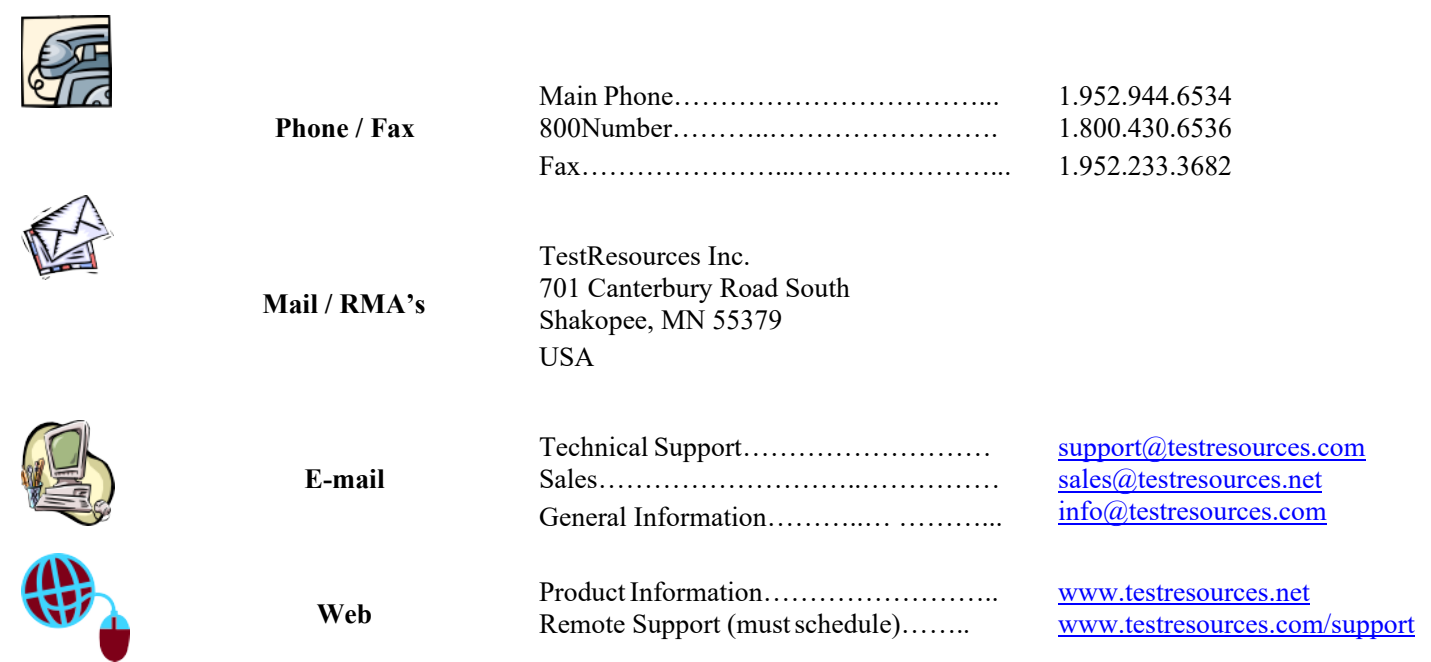

TestResources systems are engineered and constructed with care in every phase of design and assembly. To ensure reliability, mechanical and electrical components have been selected from the best components and materials. It has been proven that a thorough understanding of the system, and especially the system software, will pay big dividends. You as our customer are our most valued asset. We take pride in our systems and are proud you have become an owner. We welcome your comments about our products and wish that you express them. It is the only way that we can continue to build the best available test systems to satisfy your needs. Thank you for your support.

\subsection{General Outline of Information Related to the Product}

TestResource's Test Systems are used to generate force and measure fatigue, fracture, tensile and compression test results on mechanical test samples. They are capable of producing static and cyclic loads in tension or compression and operate to speeds of depending on the system performance. Extreme care and understanding is required for proper use.

TestResources systems are configured to order to meet specific customer requirements and use standard components where possible integrated together to meet the desired objective.

1.2 Intended Purpose and intended audience.

TestResource's Test Systems are used to generate force and measure fatigue, fracture, tensile and compression test results on mechanical test samples. They are capable of producing static and cyclic loads in tension or compression and operate to speeds of depending on the system performance. Extreme care and understanding is required for proper use.

TestResources systems are configured to order to meet specific customer requirements and use standard components where possible integrated together to meet the desired objective. Mechanical testing is an inherently dangerous activity since the product being tested may behave or fail in a way that is not expected. The nature of testing is to determine what happens when various conditions are present that have never been tested before.

In addition, servo-controlled systems, by their nature have many variables that must be factored in real time by the operator of the machine. In many cases, the person wanting to operate the machine may be lacking in training or experience and so may choose to place a sample in a dangerous mode. 
This is the nature of performing research on new products and materials. Since all materials and products fail in different modes, the customer is responsible to determine the risk and to solve the problem of minimizing it.

For example, if the material tested is brittle and fails in a catastrophic and dangerous manner, a safety enclosure is recomm ended to contain the product being tested. TestResources offers safety enclosures as products for purchase in these cases, so contact the firm for pricing information. L Series Systems components have been selected for high quality workmanship and durability, but if the system shows any sign of deterioration, contact TestResources.

Since 800 Series Electrodynamic Test Systems are capable of moving at high force and speed, only trained operators should be authorized to use the system. 
1.3 Manual List / User Manual Layout \& Explanation

It is important to understand where you can find information in the Electrodynamic System user manual. The proceeding sections explain how TestResources modular product structure is support with various user manuals. This information should allow you to determine where to find specific information related to different parts of your system such as the general information, the frame, the software, etc.

\subsubsection{Info Sheet}

Specific information related to your system can be found on the info sheet.

\subsubsection{Electrodynamic System User Manual}

Here you will find general system related information including; general testing information, and test setup information. This should be most users starting point because the information is broad enough to fit with most systems. Custom designed system information may vary slightly from the Electrodynamic System User Manual.

\subsubsection{Installation}

This manual is directed towards a technician who is settings up the system after it was shipped from TestResources. It includes site requirements, and setup instructions. Operators should study this section as well because it covers how to setup fixtures onto the machine.

\subsubsection{Operation}

This manual is directed towards the system operators. Information in this section is meant to direct the operator in how to safely use the equipment.

\subsubsection{Maintenance}

This manual in directed towards a technician who will be responsible for long term maintenance of the equipment. The system operator should also study this because there is important information regarding system maintenance that must be done on a regular basis during operation.

\subsubsection{Frame}

This manual describes mechanical features of a given frame, as well as how to perform basic setups. TestResources often builds custom frames, which may vary slightly from this manual. However, a majority of the frame operation can be cover by breaking down the frame varieties into three sections.

\subsubsection{Single Axis Axial}

This manual includes tension/compression type testing systems.

\subsubsection{Single Axis Torsion}

This manual includes torsion only testing frames.

\subsubsection{Axial Torsion}

This manual includes axial torsion systems, meaning they have both an axial actuator and a torsion motor on one frame.

\subsubsection{Planar Biaxial}

This would include systems with four axial actuator.

\subsubsection{Software}

Each system comes with a variety of software options that have specific uses. They are broken down into individual manuals with quick start and reference sections for the software. These manuals will be provided in PDF format if software programs were purchased with the system.

\subsubsection{Static}

Tension and compression testing of materials to determine stress vs strain type data.

\subsubsection{Fatigue}

Life testing of materials and products to determine or verify endurance limits. The software allows the 
system to be setup to cyclically load the specimen.

\subsubsection{MTL Programming}

Multi step testing that allows users to create custom waveforms. This is often used to replicate complicated motion that real parts see during use.

\subsubsection{MTL32_2020}

System setup and configuration software. This is used for device calibration.

\subsubsection{Fatigue Crack Growth}

This software is sold separately and is for crack growth analysis.

1.3.4.6 TestBuilder

Main user interface for system operation. 


\subsubsection{Accessories}

These manuals are sent with purchased accessories in PDF format.

\subsubsection{Oven}

Temperature controlled cambers for high temp or low temp testing.

\subsubsection{Strain extensometer}

These devices are used to measure strain directly on the specimen. This ensures high accuracy and removes machine compliance. Strain extensometers are available in clip on versions, and video systems.

\subsubsection{Grips and fixtures setup onto the machine}

Each grip/fixture has a unique application and setup requirement. 


\subsection{Safety and Training Required for Installation}

TestResources strongly recommends that the Project Manager for the purchased system is contracted to perform the system installation. This allows for correct setup and testing of the system to confirm that no damaged occurred during shipment. Many electrodynamic systems can be installed by the end user but the end user should contact TestResources technical support for assistance.

Prior to installation the system needs to be removed from the shipping container and moved to the working location. The person responsible for this first step in the installation process should read this entire installation section before starting any installation work. Specifically, the section on unpacking will be needed to prevent damage of the equipment during unpacking.

\subsection{Installation Overview}

Electrodynamic testing systems are often custom built, and will require a custom manual specifying any installation requirements. However, there are some general requirements for each system, which are covered here. Typically systems are for lab use, and can often be set of a lab table or custom built table. Check with the project manager for exact size and weight of your system.

Prior to installation, adequate space must be setup aside for the following components.

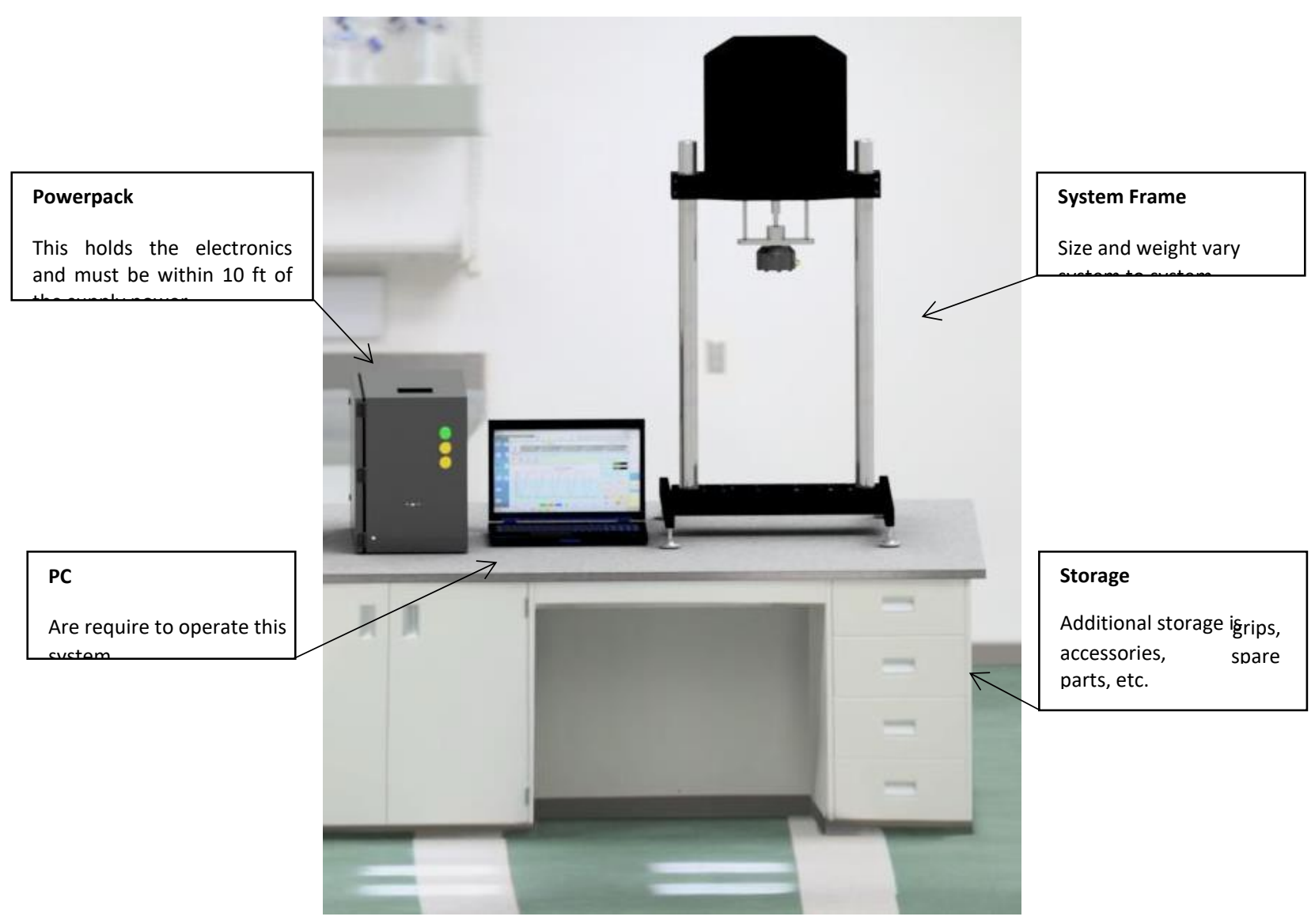




\subsubsection{Mechanical (ref. frame manual)}

Most systems come preassembled, and if additional assembly is required it will be handled by the project manager during the system installation and training. If that is not the case for your system, then detailed instructions will be sent separately, either with the machine or electronically to the main purchasing contact.

There are setup items that should still be handled prior to the arrival of the project manager for the system installation and training.

\subsubsection{Space around the machine}

The size of each system will vary greatly, however there is a need for additional space for cables to run, space for electrical maintenance, and space for operation. A general layout below shows the minimum

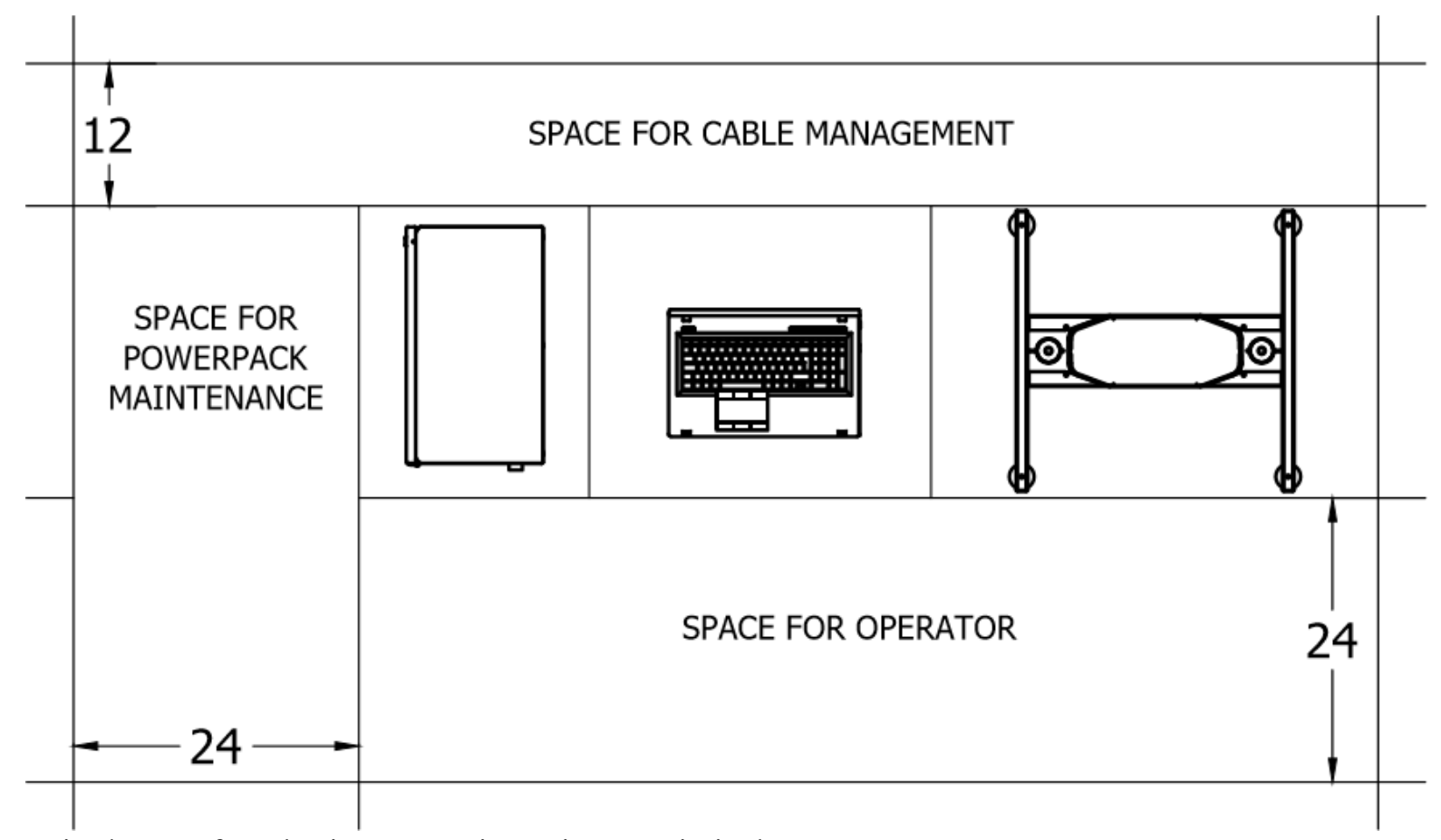

required space for a basic setup. Dimensions are in inches.

\subsubsection{Bolt torque specs and locations}

This is system/frame specific. See frame manual for more information.

\subsubsection{Leveling procedure}

This is system/frame specific. See frame manual for more information. 


\subsubsection{Electrical}

\subsubsection{Electrical connections}

Electrical connections are all clearly labeled. In general, they will go from the powerpack, shown below, to either the PC or the frame. For installation, simply connect labeled electrical connections securing to correct port. It is important to make sure connections are tight, and failure to do so can lead to issues such a noise, or arcing, depending on the connection type.

Electrical power requirements vary system to system. See the section on power requirements for more information.

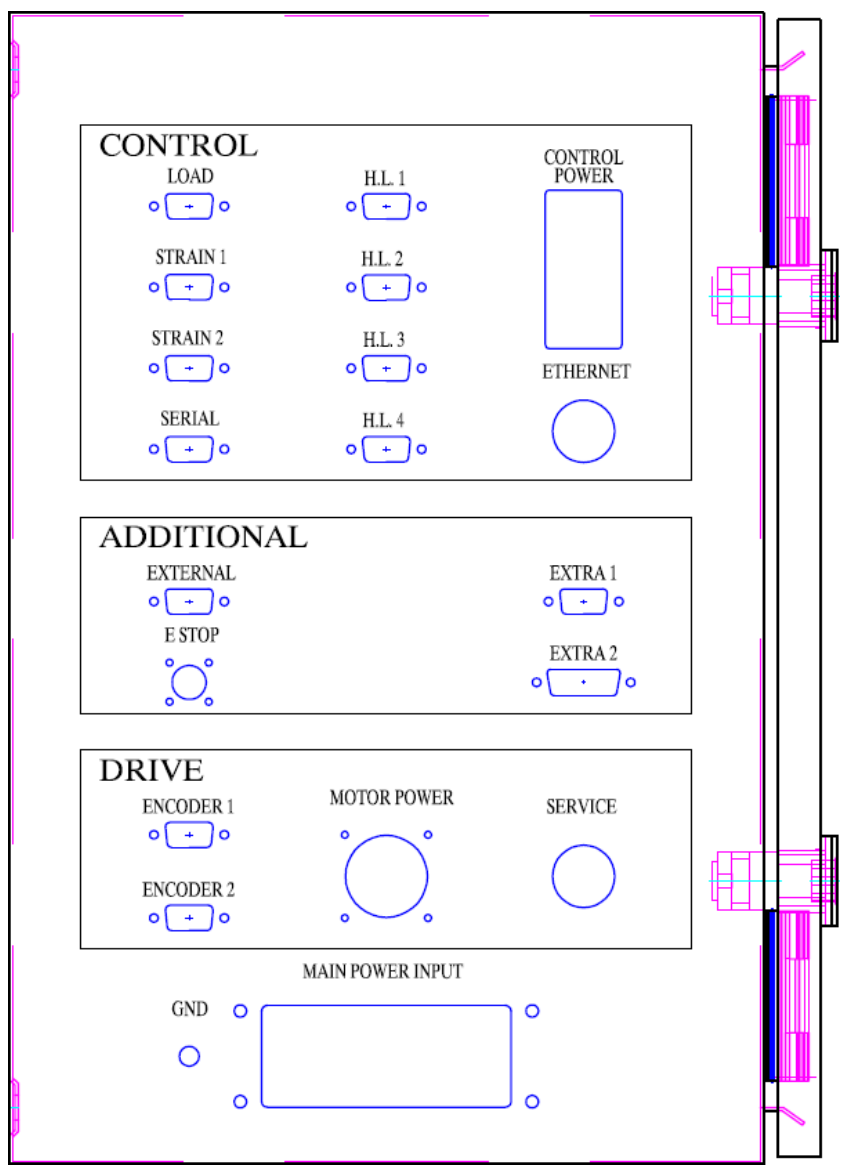




\subsubsection{Software}

For any system that includes a PC will have the software loaded on prior to shipment. In this case, installation of the software has been completed, and PC just needs to be setup and turned on.

If you did not purchase a PC with your system, you will have to follow the installation procedure in proceeding section.

\subsubsection{Installation procedure}

If you would like to have TestResources install the software for you, simply get your PC connected to the internet and contact TestResources support (support@testresources.com). They can remotely connect to your PC and do the installation for you. Keep in mind that the controller runs on an Ethernet connection, and so a second Ethernet connection is needed for the internet.

1. Insert the software CD into your PC.

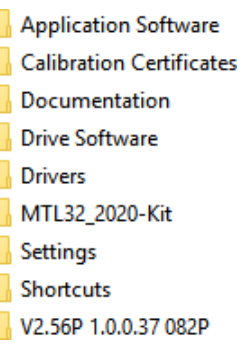

2. On the $C D$, Go into the folder Basic $>$ MTL_2020-Kit, and run setup.exe.

3. On the CD, go into Basic > "kit" (which will be something like V2.44P 1.0.0.35.080). Copy and paste all of these files into C:IMTL32 2020

4. On the $C D$, go to the Application Software folder. Copy and paste all of these files into $\underline{C}: \backslash M T L 32 \quad 2020$

5. Install additionally software applications. This includes custom software and will have specific instructions.

6. On the CD, go to the "Settings" folder and copy and paste the system settings to $C$ : $\backslash M T L 32$ 2020 Settings

7. Copy and paste shortcut folder from C: $\backslash$ MTL32 2020 Shortcuts into:

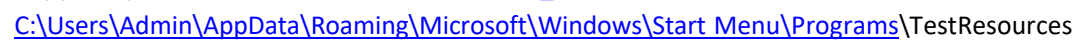

NOTE - "admin" above will be the name of the PC, and may not actually be "Admin". You will have to create the TestResources folder within the Programs folder.

NOTE - If you do not have the shortcuts, or need to set them up, use the following instructions:

a. Setup the "Properties" for the MTL32_2020 and TestBuilder shortcut.

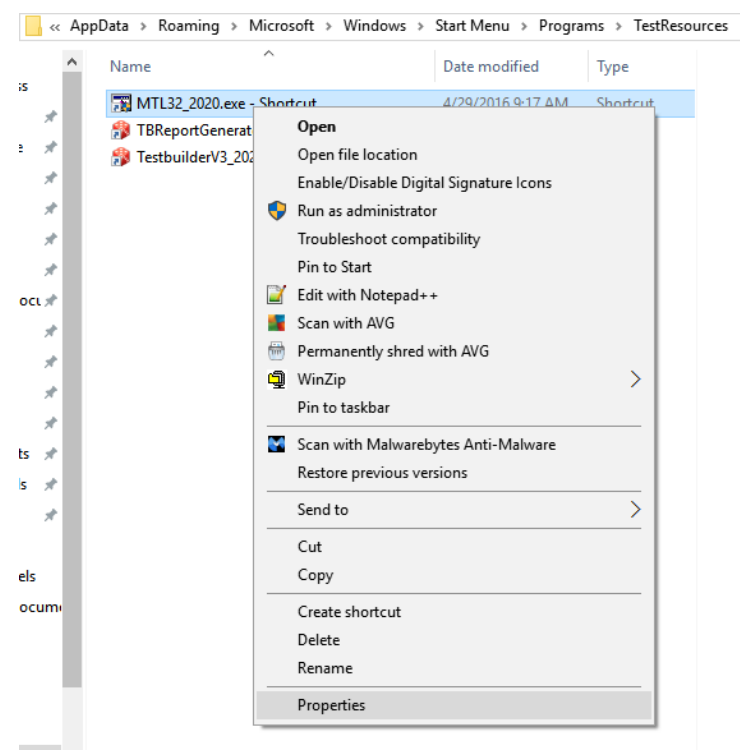


b. MTL32_2020 shortcut properties

Target $>>>>\quad$ C: $\backslash$ MTL32_2020 $\backslash$ MTL32_2020.exe "SiteID" "out file" "0 or 1" Privilege Level $\gg>>>$ Enable run this program as an administrator
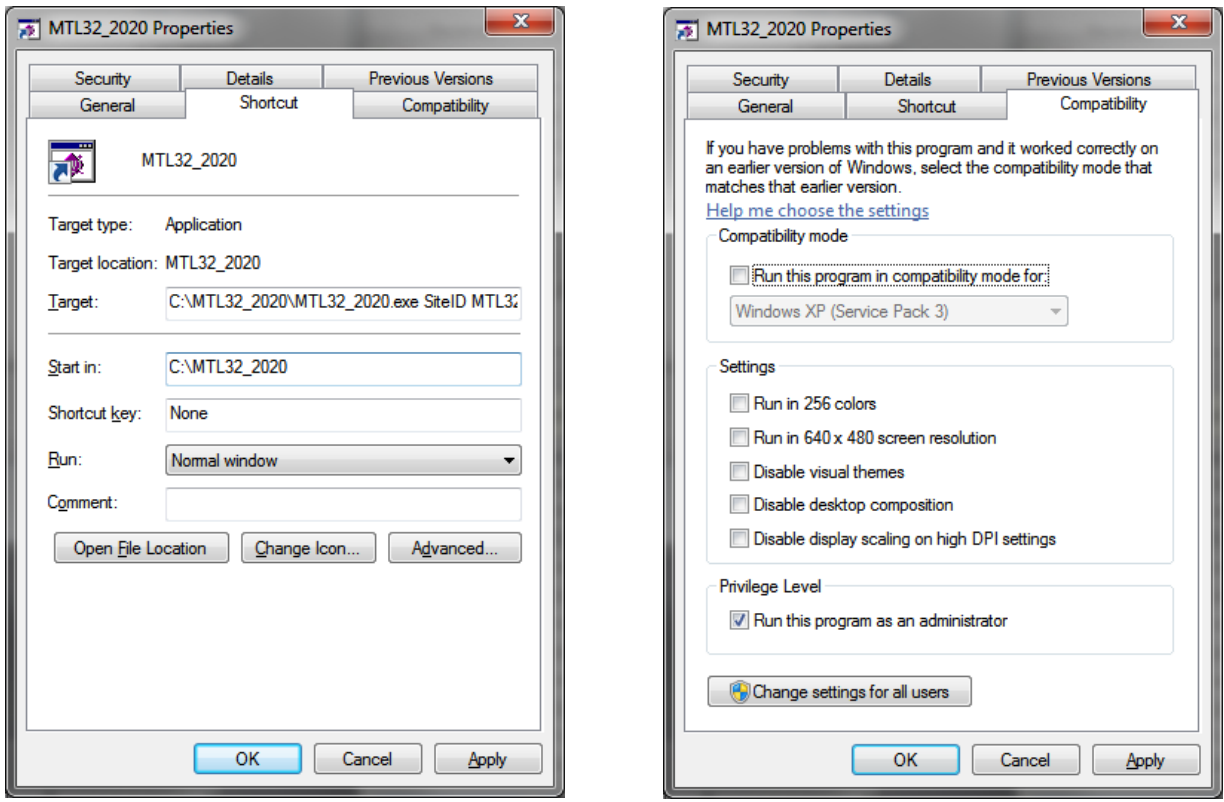

c. TestBuilder shortcut properties

Target $>>>>$ C: $\mid$ MTL32_2020 $\backslash$ TestbuilderV3_2020.exe "SiteID" "Station No"

Note - typically station no. is 0

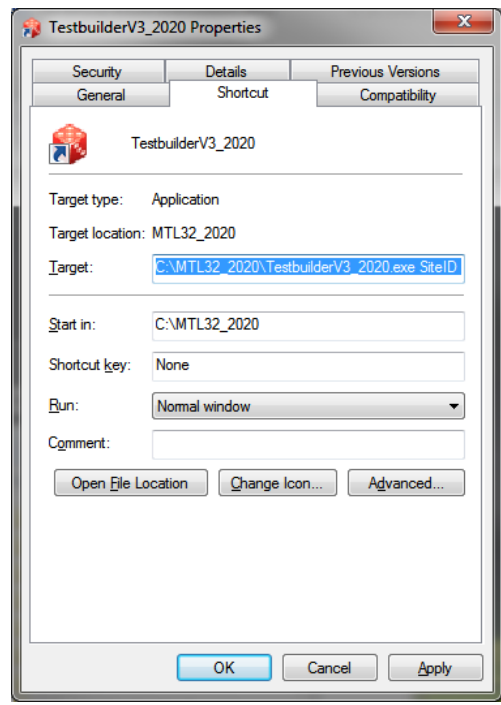

d. Pin the TestBuilder and MTL32_2020 shortcut either the desktop or Toolbar.

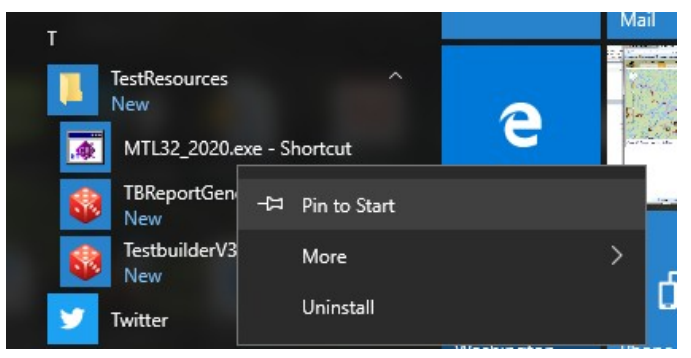

8. Install all drivers sent with the system. These are only used for support, but should be installed at this time.

9. Install the Drive Software. Again, this will only be used for support. 


\subsection{Additional required items needed to support the testing system}

\subsubsection{UPS}

The purpose of a UPS with the system is to provide power surge protection, waveform quality, and battery backup in the event of a power outage. At a minimum the PC, monitor, and controller should be connected to the UPS. Follow the instructions from the UPS manufacture for installation.

The recommended UPS model is CyberPower CP685AVR 685VA 390W.

\subsubsection{Tools}

Some necessary tools will be provided with the system, such as a torque wrench. Contact your TestResources project manager assigned to your system for more information regarding specific tools needed for your system that will not be provided.

\subsubsection{Computer Specifications and Operating System}

A PC is required to operate a TestResources electrodynamic test system. When possible it is preferred that the PC is purchased through TestResources to ensure that software, and supporting drivers are installed correctly. Another option is to send TestResources your PC for software, and driver installation. If using your own PC, it is recommended that a PC similar, or better than the following is used.

\begin{tabular}{|l|l|}
\hline Recommended PC Specifications \\
\hline RAM & $8 \mathrm{~GB}$ \\
\hline Processor & $\mathrm{I}, 3.2 \mathrm{GHz}$ \\
\hline Hard Drive & $500 \mathrm{~GB}$ \\
\hline Monitor & 19 inch \\
\hline OS & Windows 7, Windows 8 \\
\hline
\end{tabular}

2.4 Additional items that support the system, but are not required.

\subsubsection{Lighting}

Some test equipment accessories, such as video extensometers, require professional lighting to ensure accuracy in test results. Contact the test equipment manufacture or TestResources support for more information.

\subsubsection{System Enclosure}

When testing specimens that have the potential to fracture or explode, especially under stress, it is recommended that a system enclosure be installed with the testing system. Contact TestResources sales for more information regarding custom system enclosures.

\subsubsection{Cable Management}

Each electrodynamic testing system will have a variety of cables including motor power, motor feedback, main power, load cell, strain gauge, Ethernet com, serial com, etc. Once installed, most of these cables are run from the back of the PC, powerpack, and frame. When against a wall, these cables are against the wall and out of the way. However, in high traffic areas, or when cable management is important, additional cable management may be needed.

\subsubsection{Table}

Many of the electrodynamic test systems are designed to be located next to the PC on a lab table. When putting an electrodynamic test system onto a table check to ensure the table capacity will hold the weight of the system. Additionally, vibration dampening tables may be required for low force testing.

\subsubsection{Storage}

Additional space for grips, fixtures, and testing accessories will help keep the workspace around the electrodynamic system clean and organized. Contact TestResources Project Manager, or technical support for help determining what size storage may be required. 


\subsection{Electrical Power Service}

Electrodynamic system powerpacks have two power inputs; (1) controller power, and (2) main power. The control power must be run through a UPS with surge protection. The main power must be run to a dedicated circuit breaker. The main power circuit breaker should be setup within 10 feet of the system such that the main power cord will easily reach.

Required electrical power for the system can be found on the system label, which is located on the powerpack of the system. Power requirements vary system to system and therefore the system label must be checked.

\section{TESTRESÔURCES C $€$ 800 SERIES ELECTRODYNAMIC FATIGUE TEST SYSTEM}

\begin{tabular}{ll}
\hline MODEL NO. & $800 \mathrm{LKMX} 4-6$ \\
SERIAL NO. & $1603050-01$ \\
POWER RATING & $208-240 \mathrm{VAC}, 50 / 60 \mathrm{~Hz}, 1 \Phi+\mathrm{G}$ \\
CURRENT & $110-120 \mathrm{VAC}, 50 / 60 \mathrm{~Hz}, 1 \Phi+\mathrm{G}$ \\
SCHEMATIC N0. & 12 Amps (for main) \\
DATE & $40001304-02$ \\
\hline
\end{tabular}

\subsubsection{Size of upstream circuit breaker}

Service power ratings will be dictated by local regulations, but in general, the upstream breaker should be $125 \%$ the system continuous current rating. For example, if the main power continuous current rating is 12 A, then a 15 A breaker should be installed.

The system is fused as well, but a dedicated circuit breaker should still be installed.

\subsubsection{Rating: voltage, range, and frequency}

Power ratings for the system will appear on the system label. The voltage will have an acceptable range, phase (single or three), and acceptable frequency.

\subsubsection{Max power rating in amp}

The power rating of the system is given in amps. This value is the maximum continuous current rating of the system. In other words, this is the highest current draw the system pull when running at full capacity (something is not necessarily typical as you can control the motor load). 


\subsection{Installation of Mechanical Testing Accessories}

\subsubsection{Load cells}

\subsubsection{Axial Load Cells}

Axial load cells are the most common type of load cell used with an electrodynamic testing system. These load cells have a threaded base and active region, both of which have threaded holes for attachment. These can be installed onto the top or bottom of the frame.

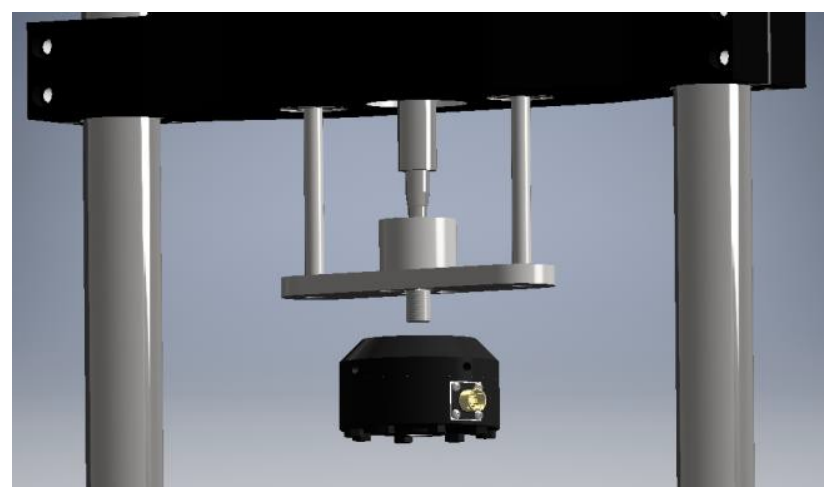

\subsubsection{Small Axial Load Cells}

Low force WMC load cells are supplied with female cup adapters for easy attachment to and from the system. Once the load cell is attached, simply snug up the spanner nuts to remove any backlash from the pin and post adapter.

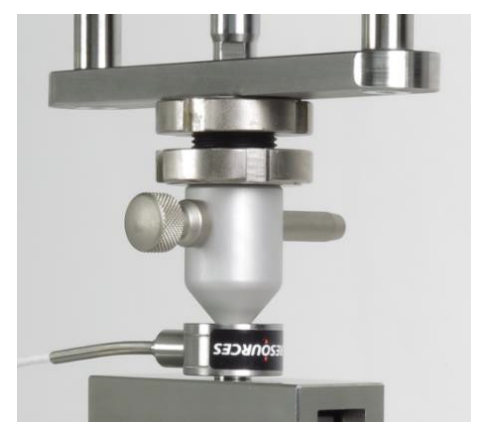

\subsubsection{Axial Torsion Load Cells}

Depending on the system, there may be additional adapters to mount the axial torsion cell to the frame. Therefore these instructions are for attaching the axial torsion load cell to either the frame, anti-rotate, or adapter.

1. Take the spacer and load cell and together, with the flange side down, attached them to the crosshead/anti-rotate/adapter using the M10x1.5 HEX bolts. Only finger tighten the HEX bolts at first.

2. Follow a star patern, and torque the M10x1.5 HEX bolts to $20 \mathrm{ft}$-lbs. It is very important to follow a start pattern when torque the bolts, or else the load cell will have some error due to uneven tightenting. 


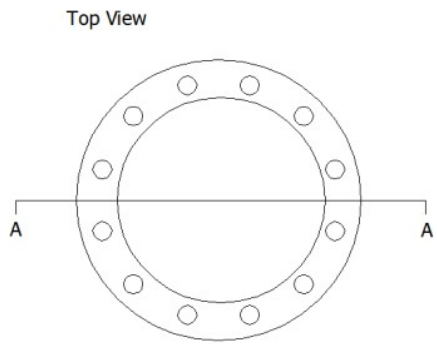

Cut View - AA

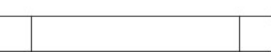

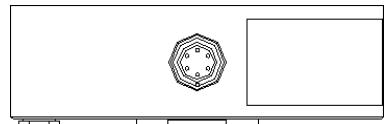

प1 
Grips and fixtures attach with a pin and post system. Lastly, a jam nut is used to remove any backlash from the fixture.

\subsubsection{Extensometers - mechanical, video, laser}

See specific user manuals for more information.

\subsubsection{Oven/furnace}

See specific user manuals for more information.

\subsection{Unpacking, and Packaging}

Electrodynamic testing systems come in many shapes and sizes, which means the factory packing will vary greatly system to system. That means that these instructions are meant to be generalities, and may not include specifics with your testing system. That being said, you should be able to unpack, and pack most systems with these instructions. If however you need additional support, contact TestResources Project Manager, or Technical Support.

\subsubsection{How to Unpack the Machine}

\subsubsection{Max angle of tilt}

Systems are designed without consideration of maximum angle of tilt. Therefore, you should never lift a system that is not upright and level, or risk it tipping and being damaged. The one exception to this is any system that is shipped laying down. In this case, the system must be tipped upright before lifting/moving.

\subsubsection{Lifting points}

Lifting points will vary system to system. Contact the project manager for more information if lifting points are not clearly defined.

2.7.1.3 Weight

Weight will vary system to system. Contact the project manager for more information.

\subsection{Final review of check-off/inspection for a correct installation}

Checklist:

Email:

support@testresources.com

Phone: 952-944-6534

1. Identify the TestResources project control number for any support calls.

2. Confirm the start and finish dates of the system warranty with the TestResources project manager. Log this information in the Quality System schedule.

3. Confirm all items on TestResources quote

4. Visually inspect all items for shipping damage. If any scratches or dents or other damage are detected, contact TestResources for appropriate resolution.

5. Confirm the machine has been assembled on site consistent with the instructions in the user manual.

6. Confirm electrical power available. See section 2.5 for where to find required electrical power.

7. Confirm the environment in the lab is reasonable room temperature and humidity.

8. Confirm the computer is situated next to the test machine such that the operator can see the test specimen while operating the software.

9. Confirm there is sufficient space in front of the test machine such that the operator can conveniently change the test specimen, change the specimen fixtures, and raise or lower the machine's crosshead.

10. During subsequent maintenance steps for machine operation verification, the machine will be operated dynamically. It will transmit small mechanical vibrations into the table and floor of the lab. Confirm there is no other equipment or instrumentation close by which would be adversely affected by these vibrations. If this is a problem, it may be possible to partially mitigate it by vibration isolation strategies. Discuss this with the TestResources project manager if necessary.

11. Confirm the load cell calibration certificate has been received. Confirm the certificate is signed and dated. File this document in the Quality System file.

12. Confirm the appropriate recalibration period for the load cell (typically 1 year). Log the recalibration deadline in the Quality System schedule.

13. Document your plan for re-calibration of the load cell on a yearly basis. If the load cell and controller are to be sent back to TestResources for recalibration, make plans for reusable packing material for shipping. File this recalibration plan in the Quality System documentation.

14. Confirm the digital encoder displacement transducer calibration certificate has been received. Confirm the certificate is signed and 
dated. File this document in the Quality System file.

15. Confirm the appropriate recalibration period for the displacement transducer (typically 1 year). Log the recalibration deadline in the Quality System schedule.

16. Document your plan for re-calibration of the displacement transducer on an annual basis. 


\subsection{Introduction with safety precautions}

Mechanical testing is an inherently dangerous activity since the product being tested may behave or fail in a way that is not expected. The nature of testing is to determine what happens when various conditions are present that have never been tested before.

In addition, servo-controlled systems, by their nature have many variables that must be factored in real time by the operator of the machine. In many cases, the person wanting to operate the machine may be lacking in training or experience and so may choose to place a sample in a dangerous mode.

This is the nature of performing research on new products and materials. Since all materials and products fail in different modes, the customer is responsible to determine the risk and to solve the problem of minimizing it.

For example, if the material tested is brittle and fails in a catastrophic and dangerous manner, a safety enclosure is recommended to contain the product being tested. TestResources offers safety enclosures as products for purchase in these cases, so contact the firm for pricing information. L Series Systems components have been selected for high quality workmanship and durability, but if the system shows any sign of deterioration, contact TestResources.

Since 800 Series Electrodynamic Test Systems are capable of moving at high force and speed, only trained operators should be authorized to use the system.

\subsubsection{Personal Protection Equipment}

When operating any mechanical testing equipment, safety glasses and steel/ceramic toe shoes are recommended to protect eyes and toes. Electrodynamic testing systems can generate large forces, enough to break bones, or crush body parts. Enclosure shields can be installed to prevent movement when performing setup on the system.

\subsubsection{Training Requirements}

To safely operate an electrodynamic testing system requires certification from TestResources.

\subsubsection{Precautions for Safe Operation}

\subsection{Operator qualifications}

TestResources Test Systems are a highly sophisticated technical piece of equipment that should only be operated by qualified or competent operators. Non-qualified and non-competent operators are at risk of getting injured by the mechanical components of the machine or more likely, damaging electrical and mechanical components of the test system. As a minimum, to operate this machine you should have experience with material testing, strong understanding of control systems, and strong understanding of Microsoft Operating Systems. Additional training is recommended to ensure damage to the operator or test system does not occur.

TestResources strongly suggests that any operator is trained by a technical support (or similar) member of the TestResources engineering team. You, the purchaser or operator, have the responsibility to get training before operating any of TestResources Test Systems. Training can be done through on-site visit from a TestResources Engineer, over the phone training, and online remote training.

Operators of TestResources 300 Series Test Systems are broken into two categories; qualified and competent. Qualified operators that those individuals who have complete understanding of the test system and can be responsible for programing new tests, running tests, grip/fixture setup, and troubleshooting problems, whereas a competent operator can be responsible for running tests, and grip/fixture setup. 


\section{Qualified Operator Prerequisite}

A qualified operator must have experience with material testing, strong understanding of control systems, and strong understanding of Microsoft Operating Systems. Additionally, they must be trained by a TestResources engineer and pass a Qualified Operator test.

\section{Competent Operator Prerequisite}

A competent operator must have a strong understanding of Microsoft Operating Systems. Additionally, they must be trained by a TestResources engineer and pass a Competent Operator test. 


\subsubsection{Qualified - must pass a test (w/ certificate)}

Within any lab, it is recommended that there is at least one qualified operator. This will ensure that there is a testing expert available for correct system operator and maintenance. This is important because the qualified operator can also serve as the main contact with TestResources for technical support, and system calibrations. Additionally, they can serve to train in competent operators as needed.

Prerequisites for a qualified operator include:

- Computer skills such as excel

- Testing experience

- Data analysis experience

- $\quad$ Engineering degree

Additionally, the qualified operator candidate must be trained by TestResources technical support/engineering. Training includes system components, safe operation, and test setup. We cover everything from load cell readings, to tuning PID control loops. Information related to wiring transducers, and tuning PID control loops is rather complicated and it is recommended that someone with an engineering degree or background be a candidate for a qualified operator.

Once trained, the qualified operator will be knowledgeable in safe system operation and will be ready to start testing.

\subsubsection{Competent - (w/ certificate)}

A competent operator is someone who will have fewer responsibilities on the testing system, but has been trained to understand safe operator on the equipment.

Prerequisites for a competent operator include:

- Mechanically inclined

- Able to operate a PC comfortably

As with the qualified operator, the competent operator must be trained by TestResources technical support/engineering. The training will center around safe operator of the equipment, running preset tests, and recording data from that test.

\subsection{Description/explanation of each hazard warning label.}

Hazard warning labels are in place to ensure operators are aware of dangers inherent to the test system. Definitions and descriptions of each hazard warning label found on the machine are shown below.

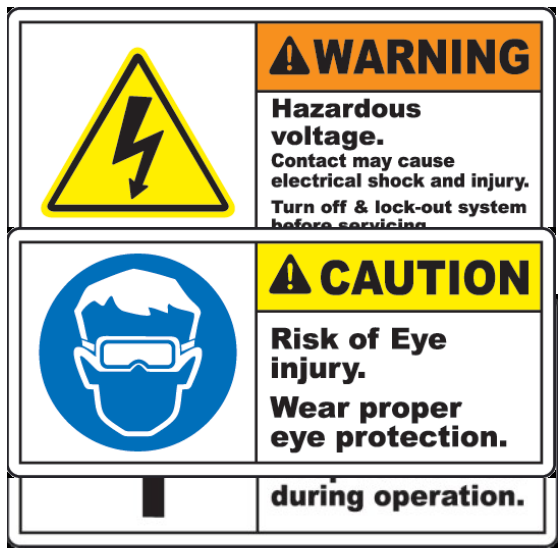

Hazardous Voltage Warning

This label indicates dangerous voltage within the powerpack. Do not open the powerpack until after the lock-out procedure is followed to turn off the machine. Only service electrical components if you are a certified electrician in the state or county in which the test system is located.

\section{Pinch Point Warning}

This label indicates a potential pinch point between the base of the test system, and the moving actuator. Keep hands clear when either the GREEN or YELLOW indication LED is on.
Risk of Eye Injury Caution

Some specimen (typically brittle material) may shatter during testing and eye protection is necessary to prevent injury. You, the operator, are responsible for determining if your specimen may shatter during testing. If you are unsure whether or not your specimen may shatter, you must wear eye protection. 


\subsection{Safety features - engineering, hazard warning labels}

There are two main concerns when it comes to operating this system; prevent damage to your person, and prevent damage to the machine. There are a number of different safety features engineered into the system design for safety, and hazard warning labels for areas that are dangerous. Before the testing begins, it is important to understand the various safety features of the machine, and the inherit dangers.

\subsubsection{System Outline}

The system shown below may not match your system, but many of the same components are used, and the terminology will apply. This diagram is meant to define various components on an electrodynamic testing system. Items related to safe system operation will be covered in the proceeding sections.
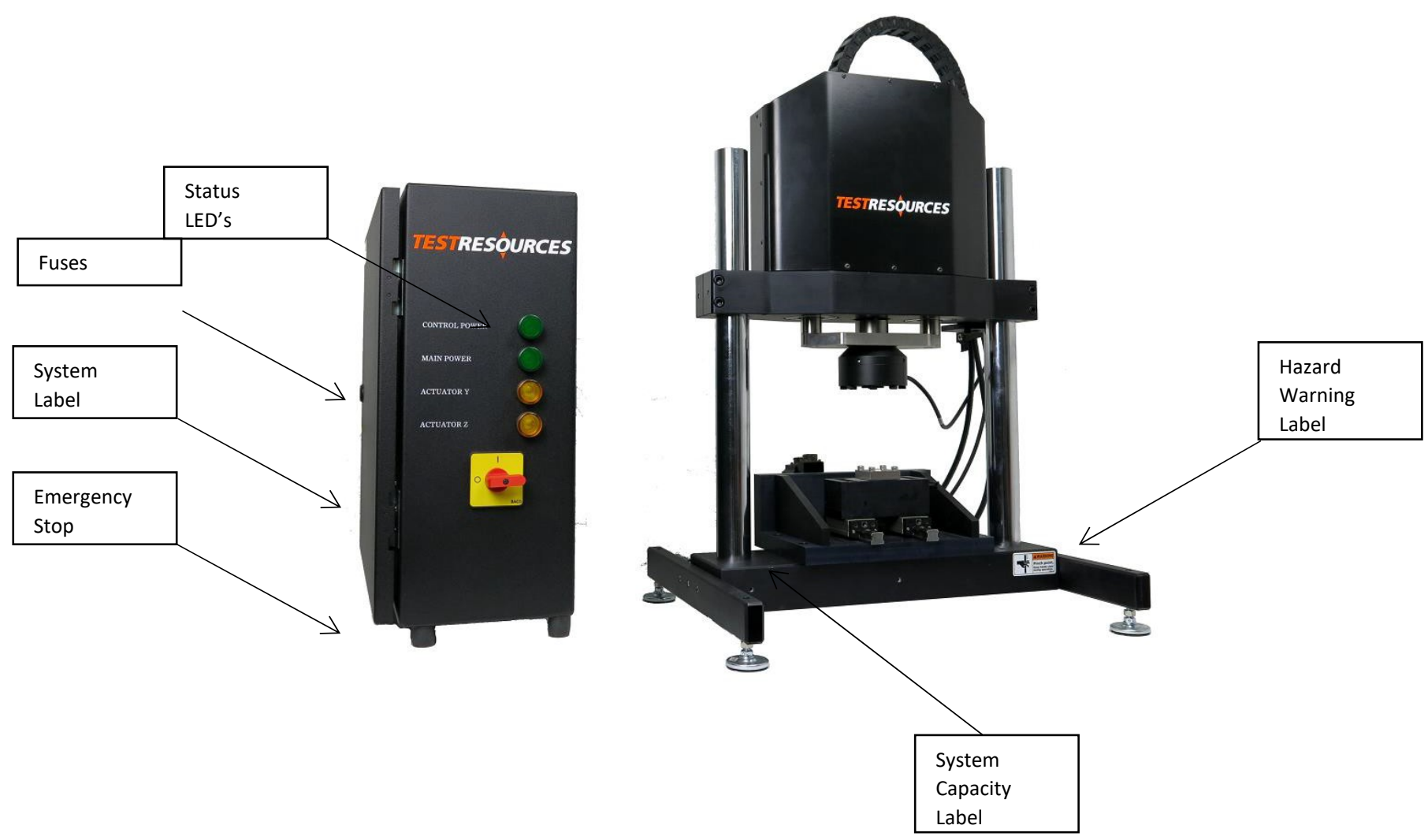


\subsubsection{Hazard warning labels}

There are three areas marked with hazard warning labels and they should be a point of concern when operating the equipment.

First, the pinch point label applies to multiple points where fingers could be crushed. When the crosshead is moving, the machine can only sense contact through the load cell. Therefore, the area between the antirotate and crosshead is a pinch point. Fingers should never be on the top side of the anti-rotate.

Second, fingers should never be on the lower side of the load cell. This location is dangerous because fingers could be pinched as the actuator moves the anti-rotate assembly down. Additionally, fragile specimens can also be damaged in this location, but the risk can be mitigated through software limits.

\subsubsection{Safety devices}

3.4.1.3 E-Stops

Both hardware and software versions of E-Stops can be found on the TestResources 800 Series Electrodynamic Test Systems.

\section{Hardware Emergency Stops}

Physical emergency stops can be found on the front on some versions of powerpacks, and on a moveable emergency stop box. The moveable emergency stop should be placed within reaching distance of the operator during all times.

Pressing any emergency stop will cut power to the actuator; this in turn cuts connection with the control system. The machine is now in a powered on state, however power is not enabled and operation is not possible, and software will be locked.

After releasing the emergency stop the state of the machine will not change, however the software will no longer be locked.

\section{Software Emergency Stops}

MTL32 and TestBuilder have emergency stops built into the toolbar. These allow operators on the computer to quickly stop the machine if a problem occurs.

Pressing any emergency stop will cut power to the actuator, this in turn cuts connection with the control system. The machine is now in a powered on state, however power is not enabled and operation is not possible, and software will be locked.

After releasing the emergency stop the state of the machine will not change, however the software will no longer be locked.

\subsubsection{Fuses}

Fuses are used to protect valuable electrical equipment from over loading. Replacement fuse size can be found labeled on or near fuse boxes in the machine.

\subsubsection{Circuit breakers}

In some cases, circuit breakers are used instead of fuses, but serve the same purpose. Additionally, building power circuits should have circuit breakers for the main power. Circuit breakers should be properly sized based on the current rating, which can be found on the system label.

\subsubsection{Status LED's}

Each testing situation is different and for that reason only our standard safety devices are listed in this manual. Per customer agreements, TestResources can provide safety shields, proximity sensors, and shields to be equip onto any test system.

Within MTL32_2020 and TestBuilder software programs operators have the ability to set motion limits of 
the machine. See the MTL32_2020 and TestBuilder manual to ensure that these limits are set properly.

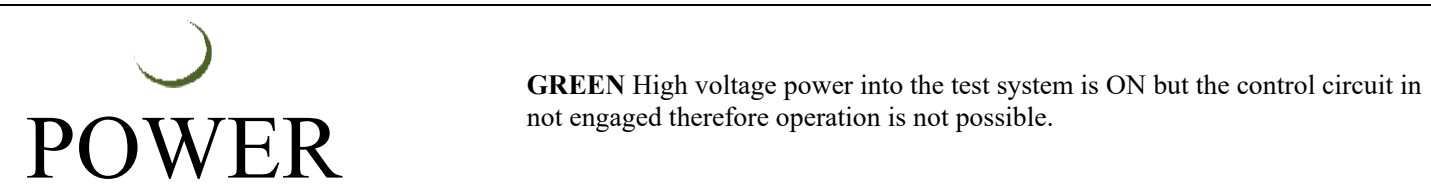

ENABLE

YELLOW High voltage power into the actuator is ON and control circuit is engaged allowing for operation. 
3.5 Equipment - describes set-up, equipment use, programing, etc.

3.5.1 System drawing with explanations of various components.

Powerpack
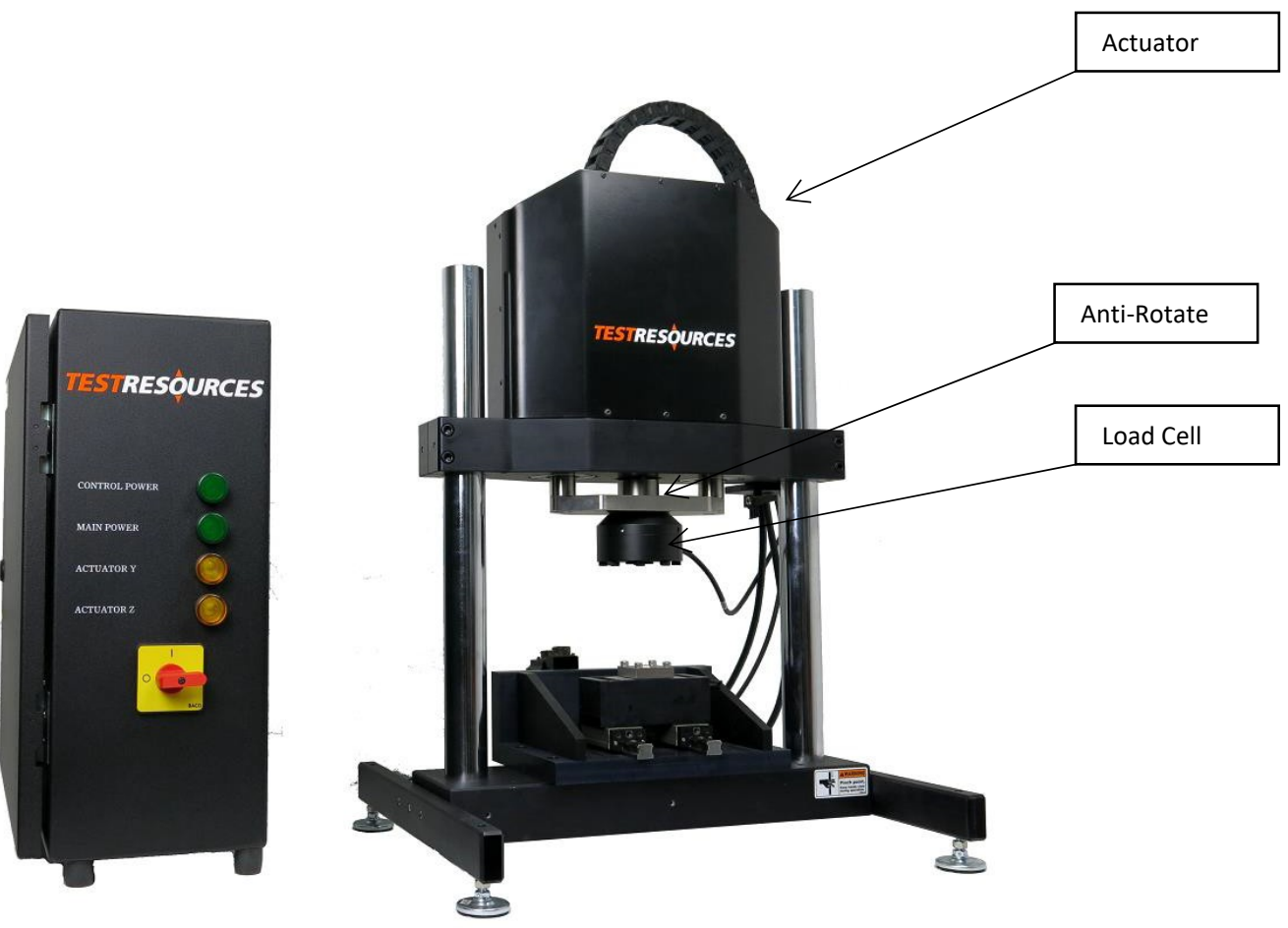


\subsubsection{Grip attachment (\& ratings)}

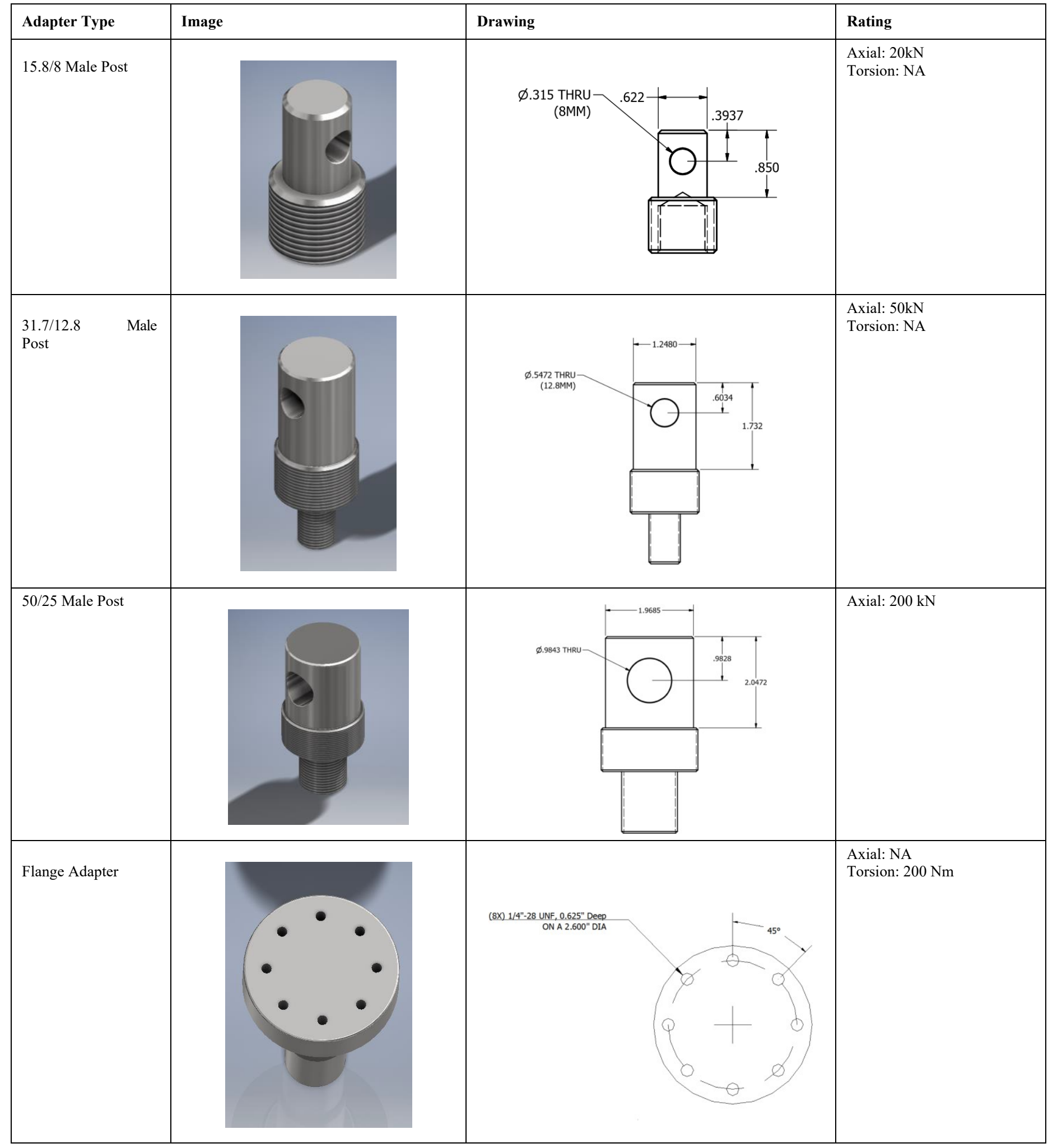




\subsubsection{Basic test setup and operation - quick start. Reference s/w manuals as needed.}

The quick start guide will step through the entire process of setting up a fatigue test, and will include pictures to assist the process. If needed, contact TestResources support for additional training.

1. Turn on Control Power and Main Power on the powerpack. The Control power is located at the back of the powerpack,and the Main Power is located at the front. Wait 30 seconds before proceeding.

2. Double-click the MTL32_2020 shortcut located on the desktop. This should take around 1 minute to open. Once MTL32_2020 opens simply leave it running in the background.

3. Double-click on the TestBuilderV3 shortcut located on the desktop. TestBuilder will connect to MTL32_2020 automatically.

4. Click on the Proceed $\gg$ button in the TestBuilder 3 window.

5. Click on the Test menu from the TestBuilder Toolbar. 


\subsection{Powerpack connections}

\subsubsection{Various electrical connections on the powerpack.}

\subsubsection{Load $(\mathrm{mV} / \mathrm{V})$}

The load cell circuit uses a shunt resistor for calibration. The shunt resistor is must match the bridge resistance of the load cell to work correctly. See example.

Powerpack Connector Type: 9 Pin D-Sub, Female (receptacle)

\begin{tabular}{|l|l|}
\hline PIN & FUNCTION \\
\hline 1 & EXC + \\
\hline 2 & GROUND \\
\hline 3 & OUT + \\
\hline 4 & OUT - \\
\hline 5 & ID CHIP (not currently used) \\
\hline 6 & EXC - \\
\hline 7 & SHUNT RESISTOR IN + \\
\hline 8 & SHUNT RESISTOR IN - \\
\hline 9 & SHIELD \\
\hline Shell & NC \\
\hline
\end{tabular}

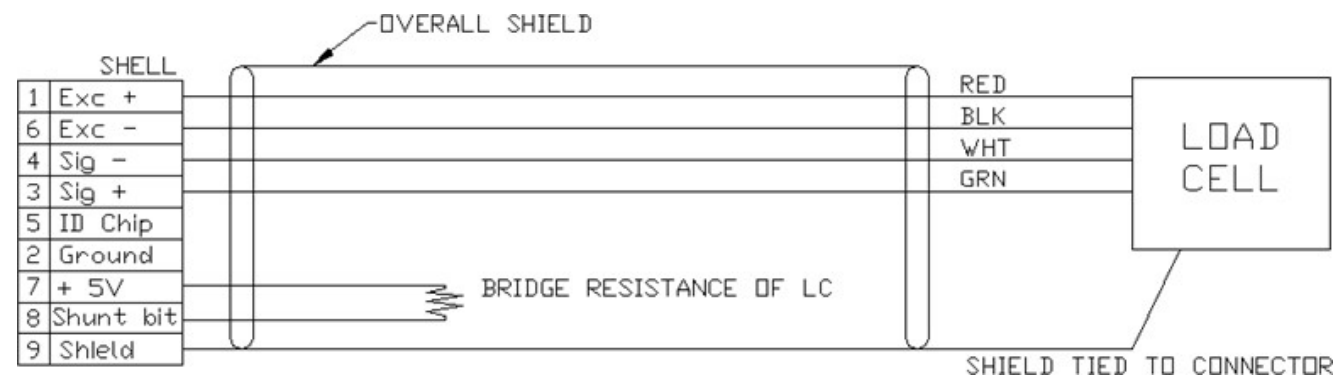

3.6.1.2 Strain $(\mathrm{mV} / \mathrm{V})$

Load and strain inputs both operate on the same circuitry.

Powerpack Connector Type: 9 Pin D-Sub, Female (receptacle)

\begin{tabular}{|l|l|}
\hline PIN & FUNCTION \\
\hline 1 & EXC + \\
\hline 2 & GROUND \\
\hline 3 & OUT + \\
\hline 4 & OUT - \\
\hline 5 & ID CHIP (not currently used) \\
\hline 6 & EXC - \\
\hline 7 & SHUNT RESISTOR IN + \\
\hline 8 & SHUNT RESISTOR IN - \\
\hline 9 & SHIELD \\
\hline Shell & NC \\
\hline
\end{tabular}

3.6.1.3 HL (Analog Input)

$\mathrm{HL}$ is an acronym for High-Level. HL inputs are analog inputs, typically $+/-10 \mathrm{VDC}$, but controllers can be modified for $+/-5$ VDC.

Powerpack Connector Type: 9 Pin D-Sub, Female (receptacle)

\begin{tabular}{|l|l|}
\hline PIN & FUNCTION \\
\hline
\end{tabular}




\begin{tabular}{|l|l|}
\hline 1 & + ANALOG INPUT \\
\hline 2 & NC \\
\hline 3 & - ANALOG INPUT \\
\hline 4 & NC \\
\hline 5 & NC \\
\hline 6 & REF. GROUND \\
\hline 7 & +5 VDC (for excitation) \\
\hline 8 & +12 VDC (for excitation) \\
\hline 9 & +24 VDC (for excitation) \\
\hline Shell & NC \\
\hline
\end{tabular}

3.6.1.4

Encoder - for Strain Measurement Only

Encoder connections are dependent on the controller type. Contact TestResources for more information. 
Powerpack Connector Type: 9 Pin D-Sub, Female (receptacle)

\begin{tabular}{|l|l|}
\hline PIN & FUNCTION \\
\hline 1 & NC \\
\hline 2 & $\mathrm{~A}+$ \\
\hline 3 & $\mathrm{~A}-$ \\
\hline 4 & $\mathrm{~B}+$ \\
\hline 5 & $\mathrm{~B}-$ \\
\hline 6 & REF. GROUND \\
\hline 7 & +5 VDC (for excitation) \\
\hline 8 & +12 VDC (for excitation) \\
\hline 9 & NC \\
\hline Shell & NC \\
\hline
\end{tabular}


3.7 Intended use of the equipment

\subsubsection{System Rated Capacity - Testing Range}

Each system has limitations on force, and speed, and these limitations must be followed. Each system has a label found on the front which shows the rated capacity of the system.

SYSTEM CAPACITY RATING

\begin{tabular}{lll}
\hline STATIC FORCE* $^{*}$ & $11,500 \mathrm{lb}$ & $(50 \mathrm{kN})$ \\
FATIGUE FORCE* & $11,500 \mathrm{lb}$ & $(50 \mathrm{kN})$ \\
SPEED MIN. & $0.001 \mathrm{in} / \mathrm{min}$ & $(0.025 \mathrm{~mm} / \mathrm{min})$ \\
SPEED MAX. & $10.000 \mathrm{in} / \mathrm{sec}$ & $(254 \mathrm{~mm} / \mathrm{sec})$ \\
STROKE LENGTH & $9.00 \mathrm{in}$ & $(203.2 \mathrm{~mm})$ \\
FREQUENCY & $15 \mathrm{~Hz}^{* *}$ & \\
\hline
\end{tabular}

* Load cell rating may be different, check load cell before operation

** Stroke dependent

\subsubsection{Disp. vs. force plots}

The performance data plot shows actuator performance at $80 \%$ continuous current draw in displacement control with no specimen load. This will allow for an approximate max displacement at a frequency, and give room for specimen loading. Amplitude is equal to half the peak to peak displacement. Use this plot as a reference when settino un hioh freauency load control fatioue/cuclic tests. Performance may vary.

\section{Amplitude [in] vs Frequency}

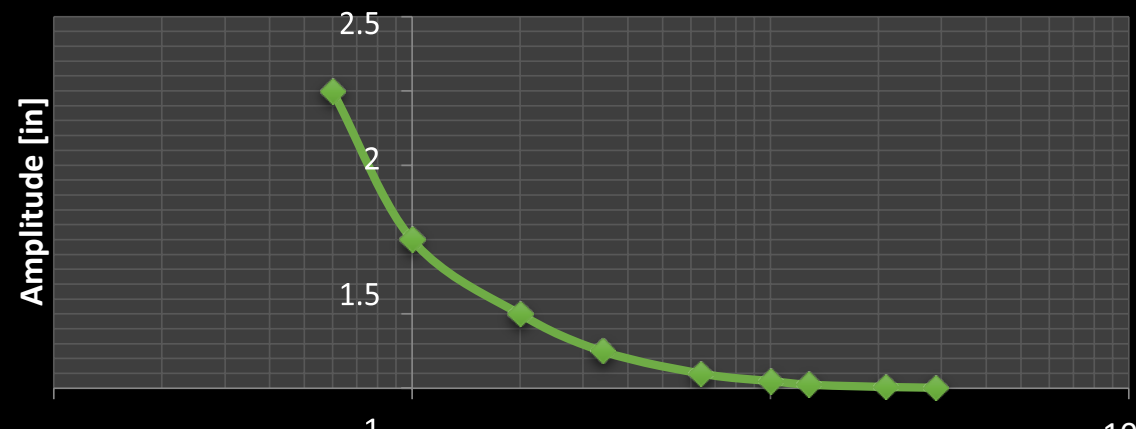

Frequency [Hz]

\section{Amplitude [mm] vs Frequency}

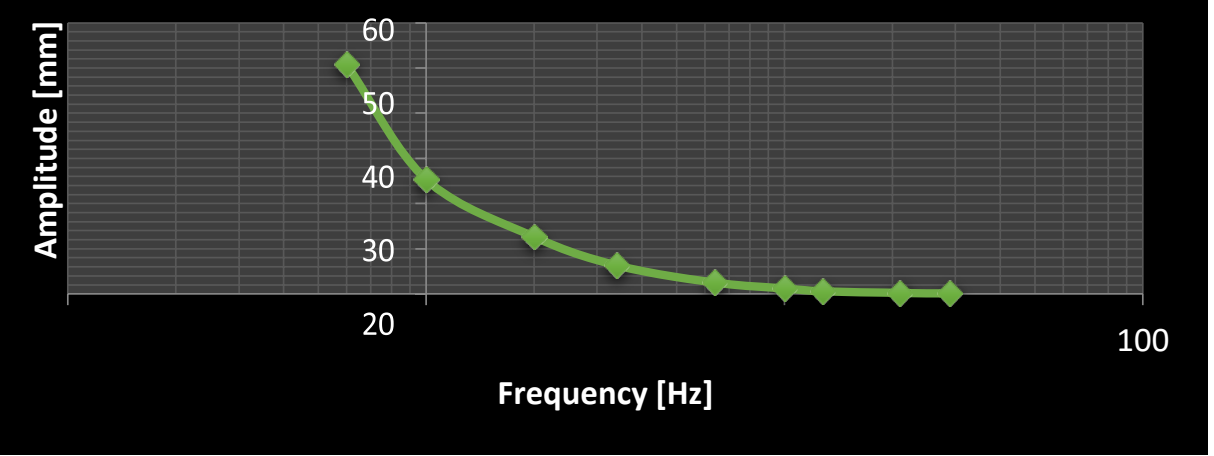




\subsubsection{PID Tuning}

Electrodynamic systems from TestResources are factory tuned for universal use. There are situations where the factory tuning parameters will not work for a specific application and need to be modified. The complexity of tuning a system can vary, but at the most basic level you will start by adjusting the coarse gain in MTL32_2020 software. This document walks through how to tune a control channel using the coarse gain of MTL32_2020. This process can be applied to Position, Load, and Strain control channels that were factory tuned and only need slight modification.

Before adjusting tuning parameters a proper diagnosis must be done to confirm the issue is in fact a tuning issue. Below is a list of situations that could require tuning the coarse gain.

\begin{tabular}{|ll|}
\hline Issue & Most Likely Solution \\
\hline Poor waveform quality & Adjust course gain \\
\hline System is vibrating (audibly) when turned on & Coarse gain is too high \\
\hline $\begin{array}{l}\text { Change to servo settings within MTL32_2020 } \\
\text { advanced settings window, or within servo amplifier }\end{array}$ & Adjust coarse gain \\
\hline Unable to reach expected test Hz & Coarse gain is too low \\
\hline Various elasticity in test specimen & Adjust course gain \\
\hline
\end{tabular}

Below is the tuning window of MTL32_2020 software. Individual parameters are defined below.

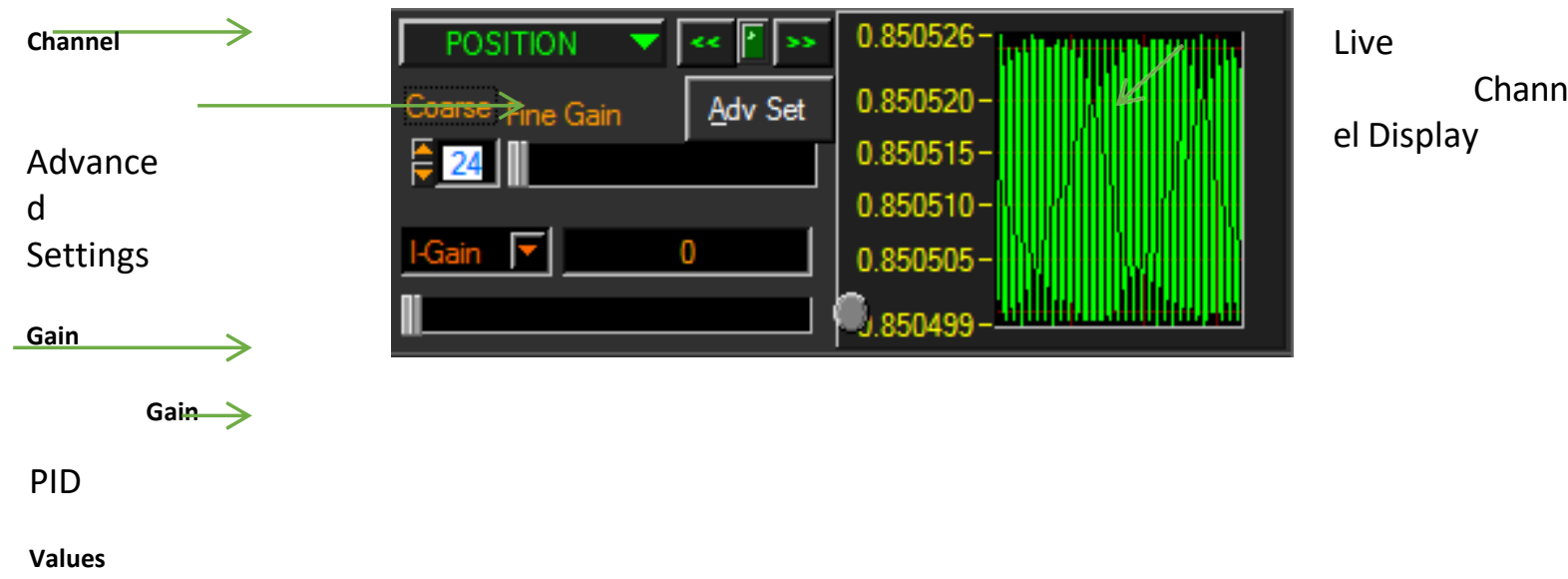

Figure 1. MTL32_2020 tuning window

\begin{tabular}{|ll|}
\hline Parameter & Definition \\
\hline Channel & $\begin{array}{l}\text { A drop down that includes all the input channels on the system controller. These include } \\
\text { Load, Strain, HL, and Encoder inputs. Each channel will have individual } \\
\text { control gains that must be independently setup. }\end{array}$ \\
\hline Advanced Settings & Do not adjust items in this window. These are factory settings only. \\
\hline Gain - Coarse \& Fine & $\begin{array}{l}\text { Coarse Gain is the main gain used on the control channel and has a significant effect on } \\
\text { system performance. Use the arrows to adjust up and down }(+/-1) .\end{array}$ \\
& $\begin{array}{l}\text { Fine Gain the slide bar is basically the decimal of the coarse gain number. If } \\
\text { Figure } 1 \text { was adjusted, all the way to the left would be 24.0, middle would be 24.5, and } \\
\text { right would be 24.99. }\end{array}$ \\
\hline PID Gain Values & Factory setup PID control gains. These do not need to be adjusted. \\
\hline Live Channel Display & Actual input from the transducer. \\
\hline
\end{tabular}




\section{Target Movement Tuning}

To tune using this technique, a basic target test is setup using the Function Generator. The following chart should be used for the test setup parameters.

\section{Control Channel}

Set Point

Rate

Position

$10-20 \%$ total range

$25-50 \%$ max rate

Load

$1-5 \%$ total range

$2 \%$ Capacity / Sec

Strain

$1-5 \%$ total range

$2 \%$ Capacity / Sec

The following shows systems responses with various coarse gain parameters. Each chart has GREEN set point and ORANGE feedback. Adjust your system according to the following charts.

Under Tuned - INCREASE the Gain by 0.25 (slider bar $+1 / 4$ ) and repeat the target movement

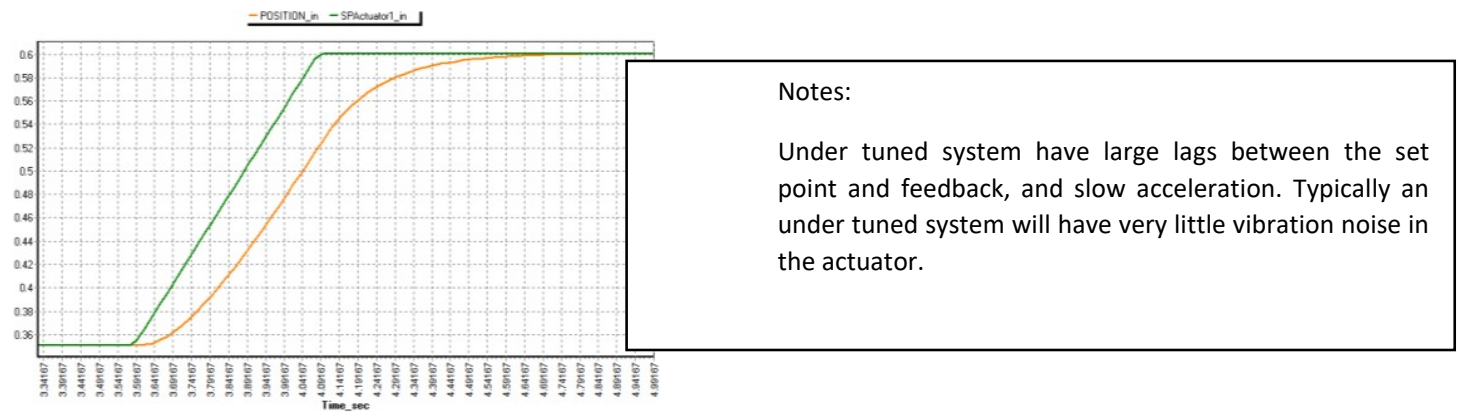

Over Tuned - DECREASE the Gain by 0.5 (slider bar -1/2) and repeat the target movement

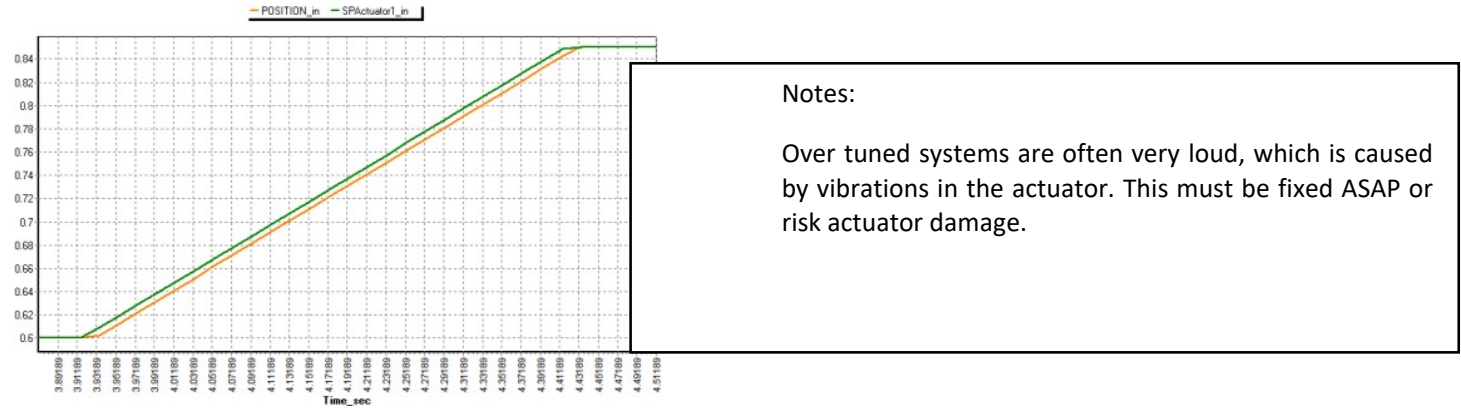

Correctly Tuned

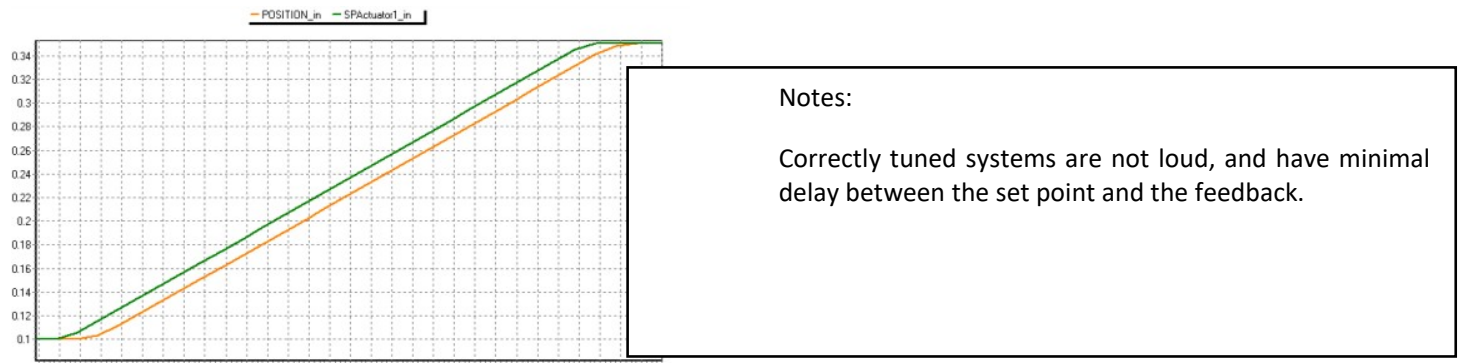

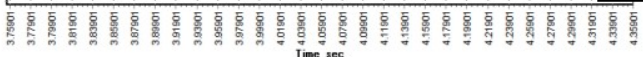




\section{Cyclic Movement Tuning}

To tune using this technique, a Cycle test is setup using the Function Generator. The following chart should be used for the test setup parameters.

\begin{tabular}{|lll|}
\hline Control Channel & Amplitude & Frequency \\
\hline Position & $1 / 2$ Motor Pitch $(0.05 \%-0.25 ”)$ & $1 \mathrm{~Hz}$ \\
\hline Load & $1-5 \%$ total range & $1 \mathrm{~Hz}$ \\
\hline Strain & $1-5 \%$ total range & $1 \mathrm{~Hz}$ \\
\hline
\end{tabular}

The following shows systems responses with various coarse gain parameters. Each chart has GREEN set point and ORANGE feedback. Adjust your system according to the following charts.

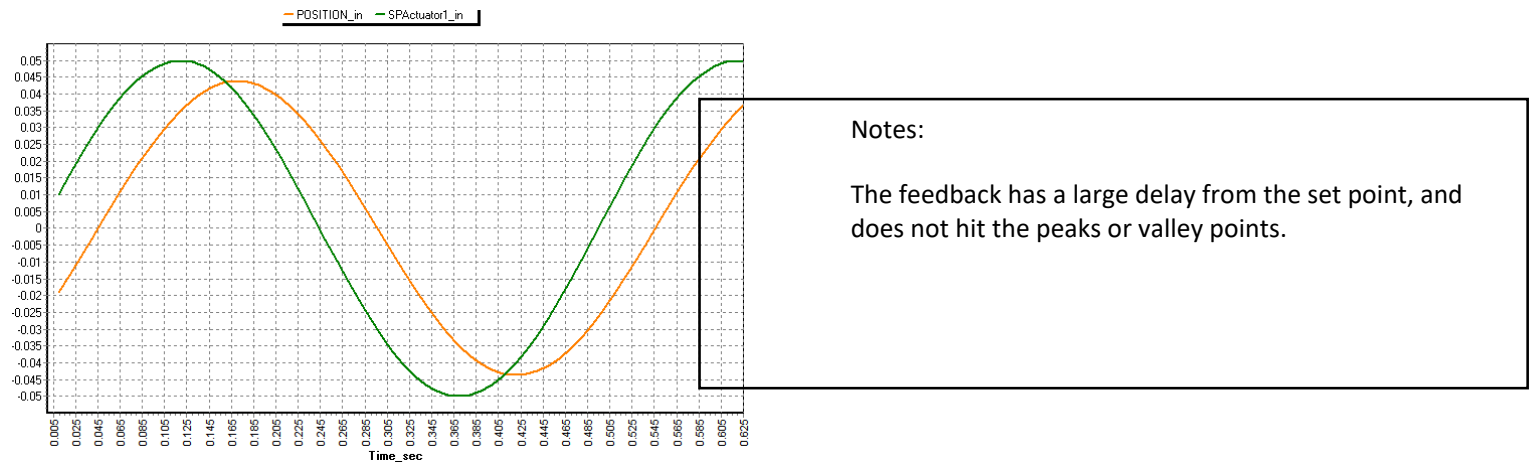

Under Tuned - INCREASE the Gain by 0.25 (slider bar $+1 / 4$ ) and repeat the target movement

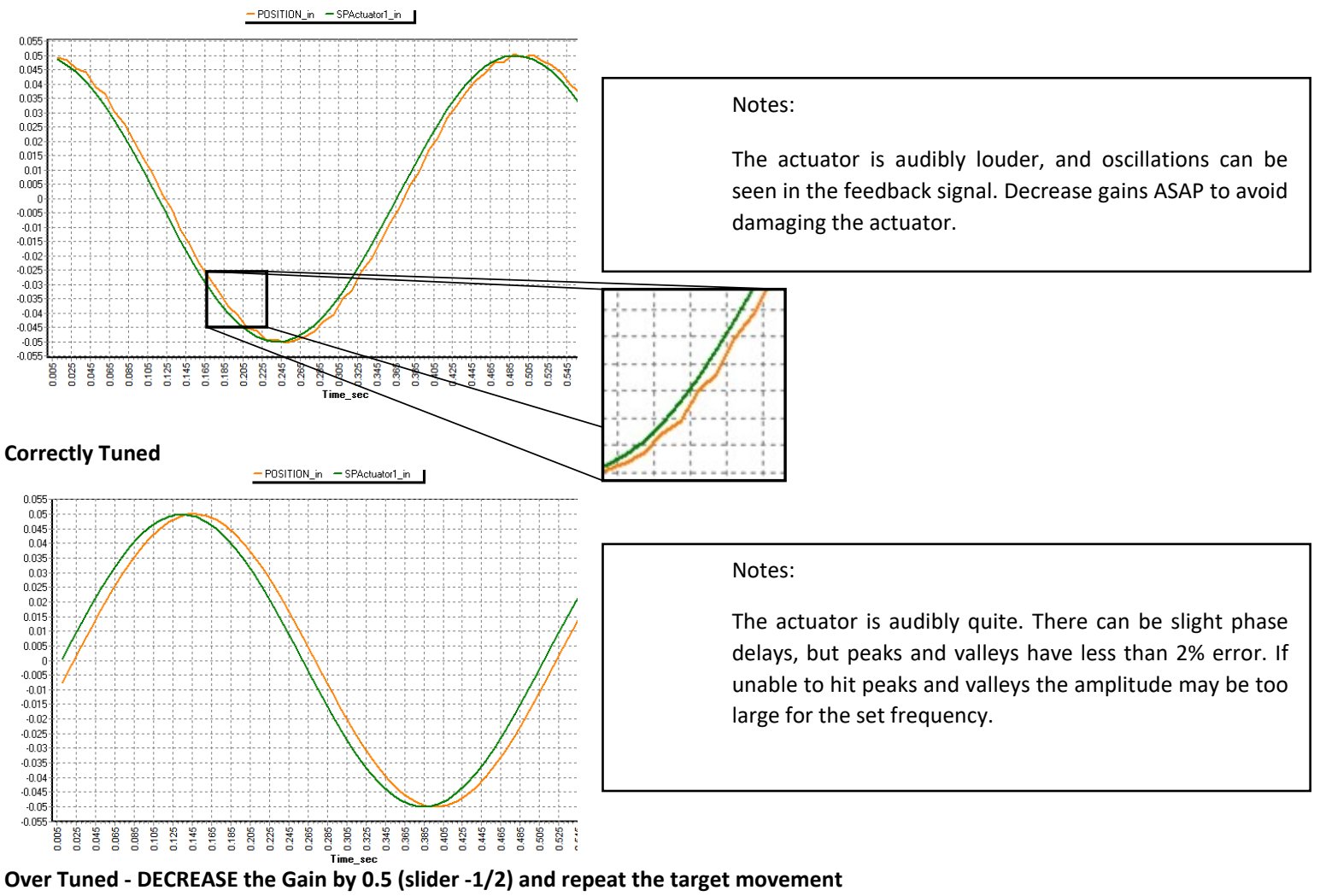


These instructions will help keep your electrodynamic system running well for as long as possible. Most electrodynamic systems are used in fatigue applications and are exposed to cyclic load forces, which will over time wear down mechanical components. By following the maintenance procedure outlined here, you will dramatically increase the life of your electrodynamic system.

4.1 Procedures/timeline for adjustment, servicing, inspection, and repair

\begin{tabular}{|l|l|l|}
\hline Maintenance Item & Reference Section & Timeline \\
\hline Operation Qualification & 4.2 & Annually \\
\hline Calibration & 4.3 & Annually \\
\hline Safety Devices & 4.5 & Quarterly \\
\hline Cleaning & 4.6 & $\begin{array}{l}\text { Harsh Environments - Weekly } \\
\text { Lab Environments - Quarterly }\end{array}$ \\
\hline Lubricating & 4.6 & 10 Million Cycles \\
\hline
\end{tabular}

\subsection{Inspection System Operation}

As part of a maintenance plan, the qualified operator should setup a basic test to verify holistic operation of the system - mechanical, electrical, and software. The following is an outline that can be used.

1. Confirm the computer boots; and you are able to start the TestResources application software program.

2. Bring the system to the ready state and confirm operation in manual control of displacement.

3. Confirmation when e-stop is press actuator cannot be enabled from software

4. Confirmation of full stroke travel in actuator. Drive limits will stop the actuator at upper and lower limit.

5. Confirmation to reach max static load.

6. With the system in the ready state, perform a shunt calibration check and confirm appropriate readout.

7. Software limits function for position and load

8. Setup a basic verification of calibration for position and load

9. Tune load and position gain settings or check / verify settings from manual or documentations received pertaining to the system.

10. Set up and run a basic fatigue and static test, gather data and confirm it exports to MS Excel or a .CSV file

11. Confirm the appropriate test operators have completed the system operation and maintenance activity. Document these names in the Quality System.

12. Confirm each operator is trained in the frequent maintenance procedure of cycling the actuator through its full travel several times before each test to ensure good distribution of the lubricant inside the actuator.

13. The test machine should be in the ready state in displacement control.

14. Operate the machine with no test specimen to confirm displacement closed loop control and stable operation of the machine:

a. Operate the machine in manual control (Jog Mode). Confirm the machine's actuator can be controlled and remains stable as needed for installation of the test fixture and specimen.

b. Operate the machine with a sinusoidal program signal at $1 \mathrm{~Hz}, 0.25$ inch double amplitude or appropriate test displacement. Confirm the machine remains stable. Confirm the force and displacement data is appropriate. (The force data will be small because there is no specimen. It will exhibit small inertial cyclicalforce).

15. Mount a dummy test specimen in the test machine. Perform a partial monotonic test to some set small force not destructive to the specimen. Confirm the force and displacement data/plot appears appropriate.

16. Run a partial cyclical test at low (nondestructive) amplitude and $1 \mathrm{hz}$ for about 500 cycles.

a. Confirm the load and displacement feedback data appears appropriate.

b. Confirm the cycle counter operated appropriately.

c. Assess the vibration transmitted around the lab, if any. If these vibrations are deemed undesirable to any nearby equipment, take appropriate mitigating action. That may include damping material under the test machine. Consult your TestResources project manager for advice if needed.

17. Manually move the crosshead and secure it at a new location. Confirm the test operators are trained as to how to do this safely with minimal risk to the load cell.

18. Confirm all trained operators are capable of performing all of the operations in this section.

\subsection{Calibration}

During system checkout at TestResources factory load, strain, torque, and position calibrations are performed. Per the calibration standard, verification of these devices needs to be done on a regular basis to check for accuracy. TestResources follows the following calibration standards with the following calibration schedule.

TestResources can be contracted for annual calibration work. We offer on-site calibrations and in many cases, equipment can be sent back for calibration at our factory. For more information contact TestResources sales. 


\begin{tabular}{|l|l|l|}
\hline Transducer & Calibration Standard & Timeline \\
\hline Load Cell & ASTM E4 & Annually \\
\hline Motor Encoder (position) & ASTM E2309 & Annually \\
\hline Strain Gauge (extensometer) & ASTM E83 & Annually \\
\hline Torque Cell & ASTM E2624 & Annually \\
\hline Speed & ASTM E2658 & Annually \\
\hline
\end{tabular}




\section{4}

Lock-Out Tag-Out Procedure

Inform the building/lab manager of the lock-out tag-out procedure. In the event of an emergency, building personnel need to be understand how to safely turn off any electrodynamic system. It is advised, that this section (or a condensed version) be posted near the system such that any person, including EMC personal, are able to turn off the system.

The following is for emergency situations only. Following this shutdown process will result in loss of testing data and unsaved items.

\footnotetext{
1. Press the emergency stop

2. Turn off main power, located on the front of the powerpack

3. Turn off controller power, located on the back of the powerpack
}

The following is for a safe complete system shut down, which should be followed when doing system maintenance.

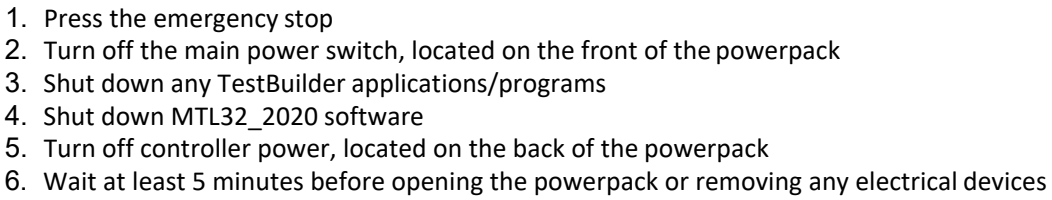

\subsection{Description and periodic inspection of the safety devices}

\subsubsection{Emergency Stops}

Both hardware and software versions of E-Stops can be found on the TestResources 800 Series Electrodynamic Test Systems. Physical emergency stops can be found on the front on some versions of powerpacks, and on a moveable emergency stop box. Pressing any emergency stop will cut power to the actuator; this in turn cuts connection with the control system. The machine is now in a powered on state, however power is not enabled and operation is not possible, and software will be locked. After releasing the emergency stop the state of the machine will not change, however the software will no longer be locked.

To test that the emergency stops are working correctly, first completely power on the system and enable the motor/actuators through TestBuilder software. The amber LED's on the powerpack will be lit when the system is enabled. Next, press the emergency stop. The system should change to a disabled state which will be indicated by a FAULT in TestBuilder software, and the amber LED will turn off. Repeat this process and check all emergency stops.

In the event that an emergency stop is not working correctly do not operate the system and contact TestResources technical support.

\subsubsection{Interlocks}

A standard electrodynamic system will have two power switches, one for the main power, and the other for the controller. To test that these switches are operating correctly, start with the system powered down. Turn the switch from off to on. Multiple indications including fans, electronics, and LED's should turn on. Turn the switch off. The indications which previously powered on will turn off.

Each electrical device in the powerpack is protected with fuses. In the event of a potentially blown fuse, start with the lock-out, tag-out procedure. Once the system is safe to inspect, open each fuse box individually and check the fuses. Visually inspect the fuse for arc marks, or damage. Additionally, use a multimeter to check that the fuse has continuity. A blown fuse should be electrically isolated end to end, therefore, if there is no continuity then the fuse is likely blown. Fuse labels inside the powerpack (often located on the inside of the door) will indicated safe replacement fuses.

\subsubsection{Panel LED's}

LED's are used with the powerpack to indicate when the system is in a powered state, and when the system is enabled. Check that LED's labeled "power" turn on when the corresponding power switch is turned on. 
Additionally, check that the LED's labeled "enable" turn on when the channel is enabled through TestBuilder. If LED's are not working, replace LED bulb by unscrewing the front colored cover of the LED.

\subsubsection{Software safety's}

Ensure that the system is able to boot up, and connect to the software without any hookup failures. Additionally, the system should be able to sit idle for multiple days without issues. If hookup failures occur, contact TestResources support for help.

\subsection{Cleaning and Lubricating}

Electrodynamic systems from TestResources are designed for fatigue testing, however care must be taken to ensure the equipment does not fail due to fatigue itself. This is the single most important information for system maintenance. Mechanical components in the actuator will wear over time, and this is accelerated from small movements, such as those done during cyclic loading. Over time, mechanical components in the actuator push grease away from surfaces in contact. For small movement cyclic tests, this can produce metal on metal contact after an extended period of time, and this is the cause of accelerated wear.

To prevent accelerate wear of the mechanical components within the actuator need to have grease redistributed across all surfaces. This is done by moving the actuator through its full stroke. This should be done, at a minimum, every 10 million cycles. 


\subsection{Troubleshooting procedures}

\section{Common Issues}

\begin{tabular}{|c|c|}
\hline Observed Problem & Possible Cause \\
\hline Unable to connect to the controller & $\begin{array}{l}\text { - IP address is incorrect. (see section 4.7.1) } \\
\text { - } \quad \text { DSP card has failed and must be replaced. }\end{array}$ \\
\hline $\begin{array}{l}\text { Occasional Communication disruption (Hookup Error in } \\
\text { MTL32_2020) }\end{array}$ & $\begin{array}{l}\text { - UPS with surge protection is not being used } \\
\text { - } \quad \text { PC firewall is stopping data stream } \\
\text { - } \quad \text { PC sleep/hibernate settings are not disabled }\end{array}$ \\
\hline Actuator grinding & $\begin{array}{l}\text { - Tuning is too hot, and the actuator is rattling. Turn down the gains. } \\
\text { - The actuator has mechanical damage. The actuator must be replaced. }\end{array}$ \\
\hline Poor performance & $\begin{array}{l}\text { - If in load control, check that all connections in the load train (grips, } \\
\text { adapters, load cell) are tight. Loose connections are backlash and it is } \\
\text { difficult to have good control when there is backlash in the load train. } \\
\text { The gains are not correct. See section 3.7.3 Tuning for more information. }\end{array}$ \\
\hline No load cell reading & $\begin{array}{l}\text { - } \quad \text { Load cell is not plugged in. } \\
\text { - Load cell settings in MTL32_2020 where changed. } \\
\text { - } \quad \text { Load cell shunt in MTL32_2020 must be reset. } \\
\quad \text { Load cell is damaged. }\end{array}$ \\
\hline Spikes in the input channels (load, strain, HL, etc.) & $\begin{array}{l}\text { - Ground issue. Check motor power cable shields are securely tied to } \\
\text { ground. Also check shield on input channel cables. Lastly, ensure the } \\
\text { frame is tied to the powerpack ground lug located at } \\
\text { the back of the powerpack. }\end{array}$ \\
\hline
\end{tabular}

\subsubsection{Problems Connecting MTL32_2020 to the Controller}

1. Check the IP address setup on the PC.

a. Connect the PC to the controller serial port. This should be done using a USB to serial adapter cable provided by TR.

b. Go to Start $=>$ Network and Internet $=>$ Network and Sharing Center $=>$ Change adapter settings $\Rightarrow>$ right click on the Local Area Connection and select properties $=>$ Double click Internet Protocol Version (TCP/IPv4)

C. The IP address in the IPV4 should have the same domain as the controller.

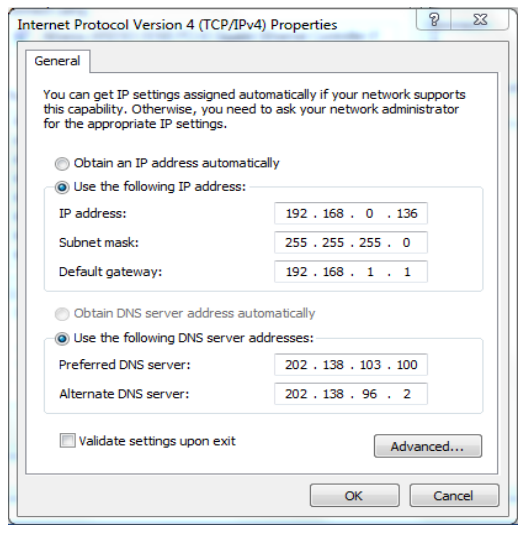

If you have the port settings correct, and still cannot connect, try to PING the controller (step 2) or check the controller IP address with Putty (step 3).

2. PING the controller from the command prompt.

a. Power up the controller

b. To open the command prompt window: Go to start $=>$ type run in the search program and files box $=>$ click on run $=>$ the run window appears. In the open box type cmd and press OK.

c. Ping the IP address of the controller. 


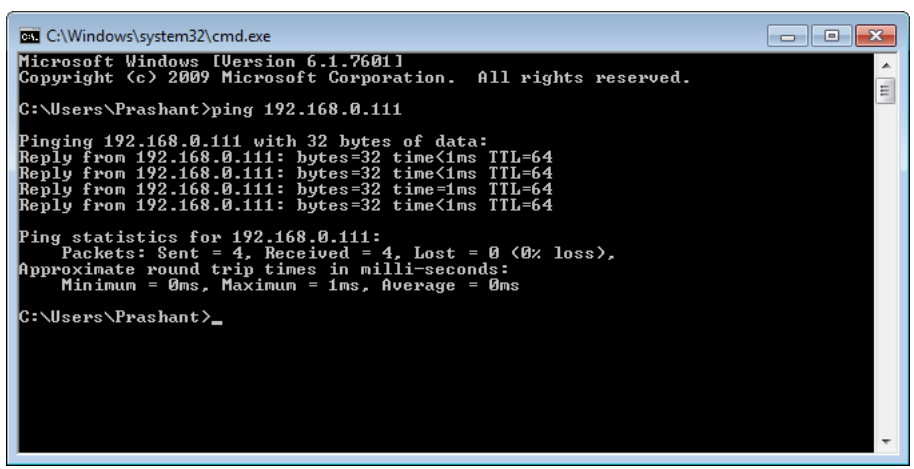

If you can ping the controller, the communication issue is likely due to PC Ethernet port settings. If you are not able to PING the controller, it may be due to 1) the wrong IP address is being ping'ed, 2) the DSP card has failed, and 3) other hardware issue.

3. Check with Putty

a. Connect serial connection to controller, and power on controller.

b. Go to DEVICE MANAGER, and check the port that the USB to serial adapter is connected to. In this example, it would be COM37

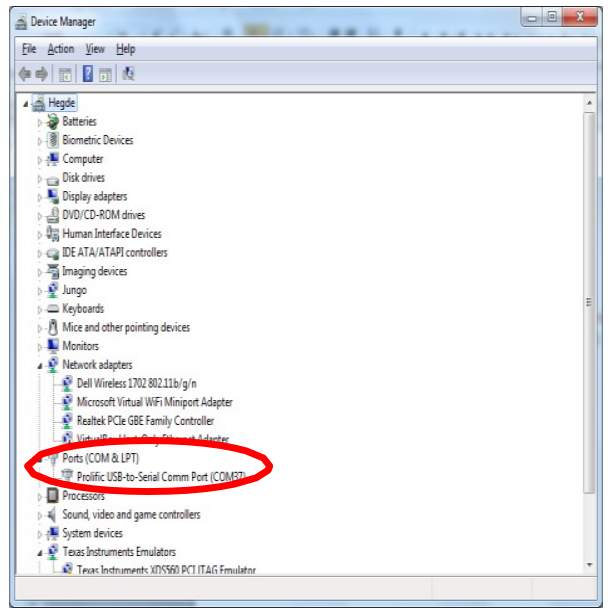

c. Open putty. C: $\quad$ MTL32_2020 \putty.exe. Be sure to right click and Run asAdministrator.

d. Ensure Putty serial port settings match Device Manager port settings for serial adapter.

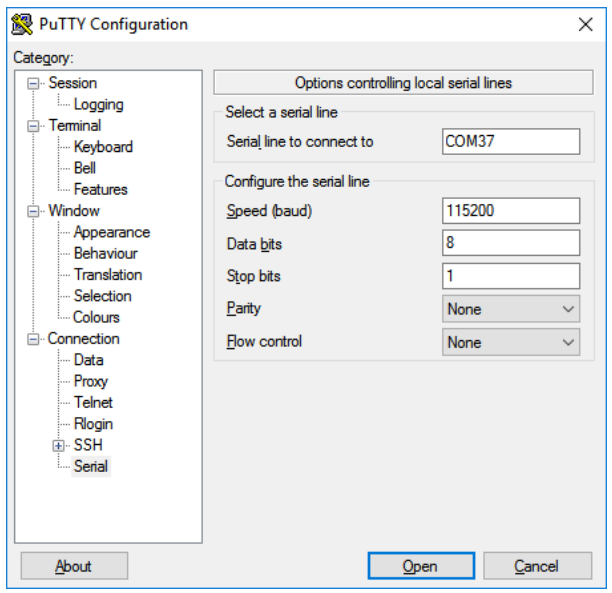

e. Save the settings.

f. Click OPEN to connect to Putty. Window below will open.

g. Power up controller (or restart power)

h. A bootup process should follow.... 


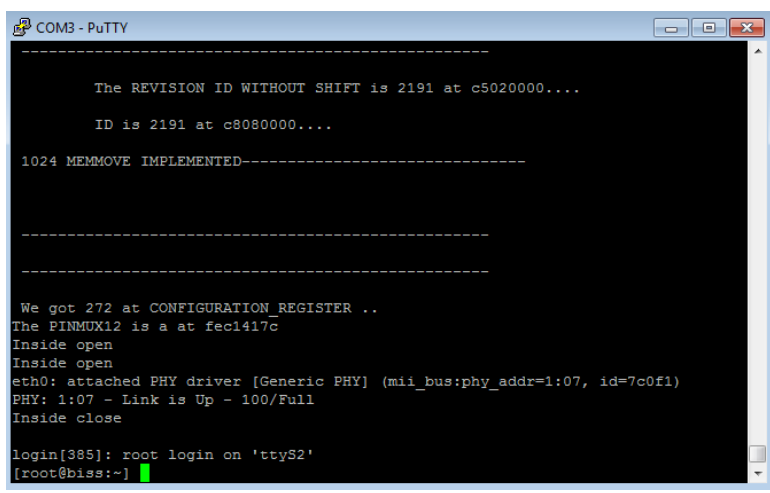

i. Once boot up is complete, press enter on keyboard. It should display [root@biss: ]. If not, report back to TR. Send screen shot of putty.

If no uses with putty, check the IP address, and verify back with PC IP domain setup (set 1), and ping controller (step 2). NOTE, to check IP address, type "ifconfig" into putty. It will return the controller IP address.

\subsection{Safe use to lift equipment}

In general the equipment, specifically the frames, should be lifted with a fork lift. If frames have i-bolts, best practice is to lift the frames with those i-bolts. If they do not, it is advised that you contact TestResources for a best method of lifting a frame.

4.9 Special tools and equipment to test problems, and inspections

\begin{tabular}{|l|l|}
\hline Item & Use \\
\hline Calibrated Multimeter & Check continuity, resistance, current, and voltage of various components. \\
\hline Small screw driver & Unscrewing electrical connections \\
\hline
\end{tabular}

\subsection{Suggested spare parts}

The following is recommended.

\begin{tabular}{|l|l|}
\hline Item & Risk \\
\hline Load Cell & $\begin{array}{l}\text { Load cells can be overloaded and damaged. The only way to repair them is to replacement. This } \\
\text { can take weeks if not already in house. New load cells will need to } \\
\text { be calibrated before use, and this can be done by local calibration companies within a couple } \\
\text { days. }\end{array}$ \\
\hline Electrical relays & $\begin{array}{l}\text { These components have a failure rate of roughly } 1 \% \text {, and if they do fail the machine } \\
\text { will not work. They low cost, and easy to replace. TestResources does stock and will ship same } \\
\text { day as needed. }\end{array}$ \\
\hline Fuse & $\begin{array}{l}\text { Over current to a system may blow a fuse. These can be purchased from multiple } \\
\text { different companies. Powerpack's all have labels for fuse replacement. }\end{array}$ \\
\hline
\end{tabular}

\subsection{Circuit diagram (schematics) for repair and troubleshooting}

All Electrodynamic systems are custom built, and for that reason, all have custom wiring schematics. TestResources log's each wiring diagram for each system, and it is available upon request.

\subsection{RMA}

In the event of a failure on a system TestResources recommends that the system be send back to our factory for diagnosis and evaluation. In some cases on-site repairs can be done, but often these are very expensive and require multiple trips. Contact TestResources Sales, or Support to get an RMA number, and instructions. Prior to shipping your system to TestResources a PO is required for the diagnosis and evaluation. The cost is $\$ 350$, and is included in the cost of any repair work.

See section on packaging to ensure safe transport to TestResources. TestResources is not responsible for safe transport or insuring equipment against damage when shipped to TestResources. 
